INTER NATIONAL MONETARY FUND
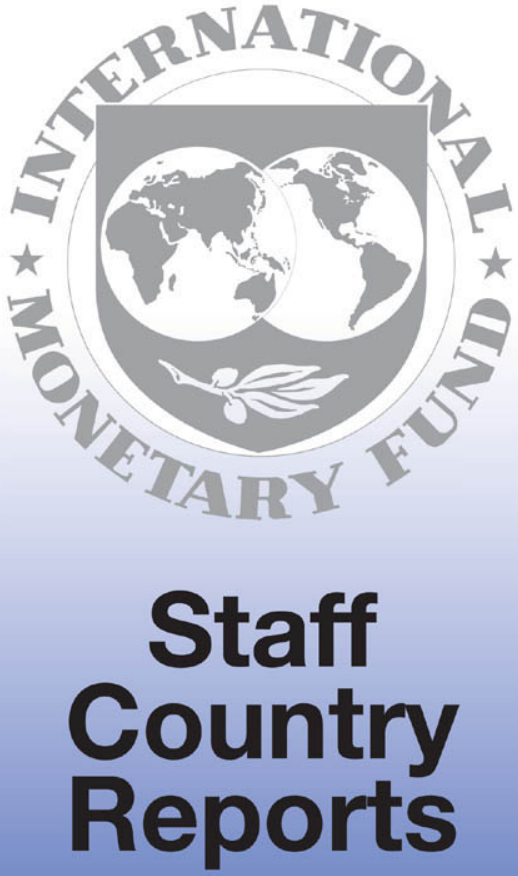


\section{Romania: Third Review Under the Stand-By Arrangement and Request for Waiver of Performance Criterion-Staff Report; Staff Supplement; Press Release on the Executive Board Discussion; and Statement by the Executive Director for Romania}

In the context of the Third Review Under the Stand-By Arrangement and Request for Waiver of Performance Criterion, the following documents have been released and are included in this package:

- the staff report for the third review under the Stand-By Arrangement and request for waiver of performance criterion, prepared by a staff team of the IMF, following discussions that ended on February 19, 2003, with the officials of Romania on economic developments and policies. Based on information available at the time of these discussions, the staff report was completed on April 10, 2003. The views expressed in the staff report are those of the staff team and do not necessarily reflect the views of the Executive Board of the IMF.

- a staff supplement of April 24, 2003 updating information on recent developments.

- a Press Release summarizing the views of the Executive Board as expressed during its April 25, 2003 discussion of the staff report that completed the request and/or review.

- a statement by the Executive Direetor for Romania.

The document(s) listed below have been or will be separately released.

Letter of Intent sent to the IMF by the authorities of Romania*

Memorandum of Economic and Financial Policies by the authorities of Romania*

Technical Memorandum of Understanding*

*May also be included in Staff Report

The policy of publication of staff reports and other documents allows for the deletion of market-sensitive information.

To assist the IMF in evaluating the publication policy, reader comments are invited and may be sent by e-mail to publicationpolicy@imf.org.

Copies of this report are available to the public from

International Monetary Fund $\bullet$ Publication Services

$70019^{\text {th }}$ Street, N.W. - Washington, D.C. 20431

Telephone: (202) 623-7430 - Telefax: (202) 623-7201

E-mail: publications@imf.org • Internet: http://www.imf.org

Price: $\$ 15.00$ a copy

International Monetary Fund

Washington, D.C. 


\title{
ROMANLA
}

\section{Third Review Under the Stand-By Arrangement and Request for Waiver of Performance Criterion}

\author{
Prepared by the European I and \\ the Policy Development and Review Departments \\ (In consultation with other departments) \\ Approved by Susan Schadler and Liam P. Ebrill
}

April 10, 2003

Contents

Page

I. Introduction 3

II. Recent Economic Developments and Performance Under the Program..............................4

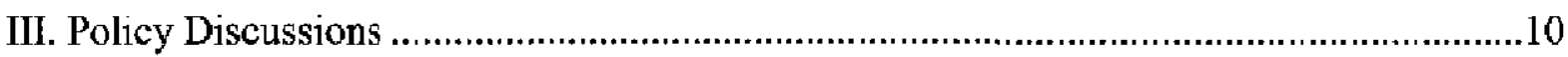

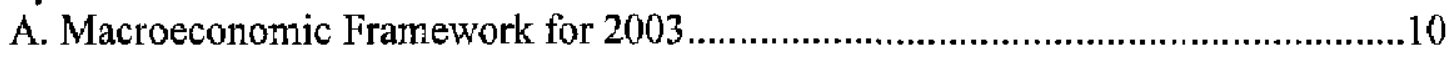

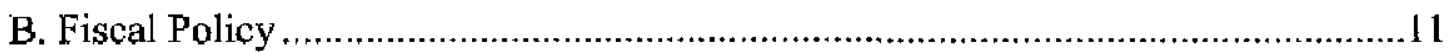

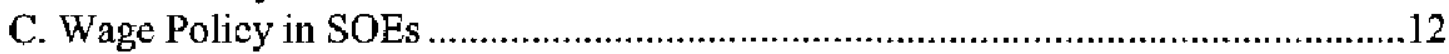

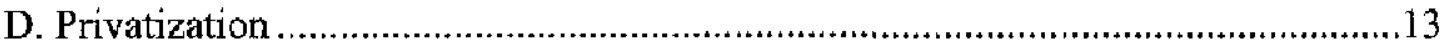

E. Financial Discipline and Energy Sector Reforms ............................................13

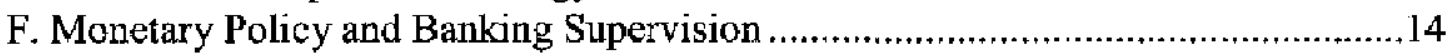

IV. Medium-Term Outlook, External Vulnerability, and Capacity to Repay the Fund ..........15

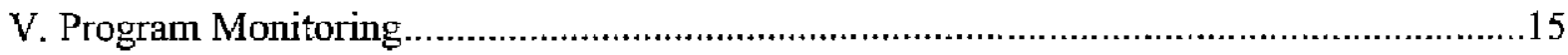

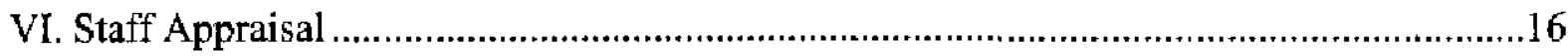

Text Tables

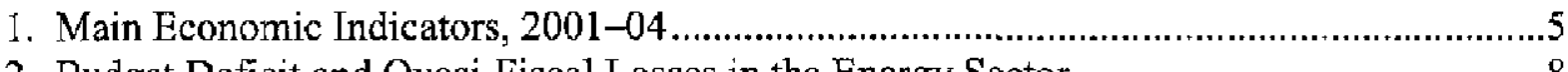

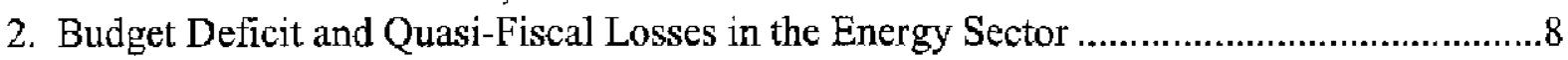


Figures

1. Industrial Production and Exports, 2000-03

2. Trading Partners' Imports Demand and Romania's Exports 2000-03 _..........................4

3. Consumer Price Index (CPI) and Net Wages, 2000-03 ..........................................

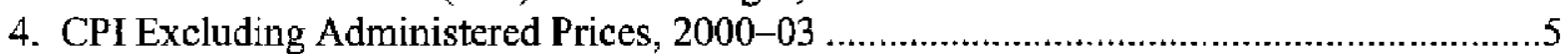

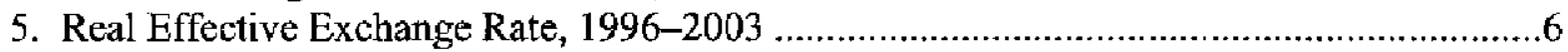

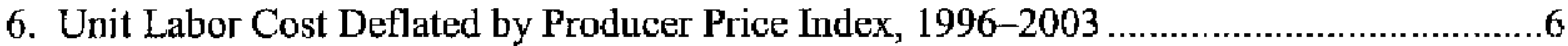

7. Reserves, Exchange Rates, and Bond Spreads, 2000-03 _............................................

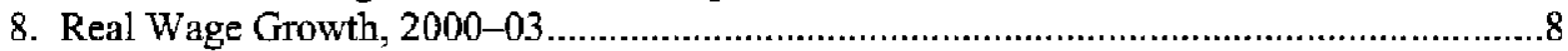

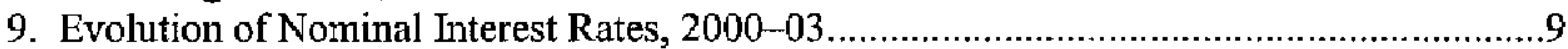

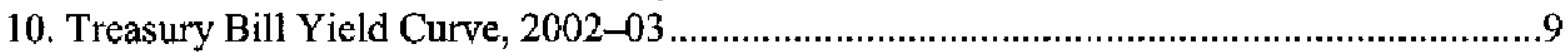

Tables

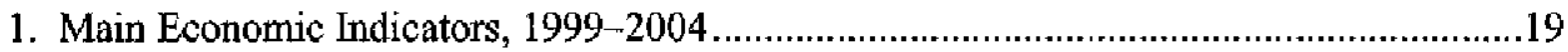

2. Balance of Payments, 2002 -03 ….............................................................20

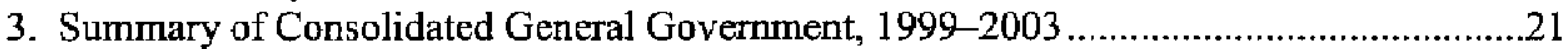

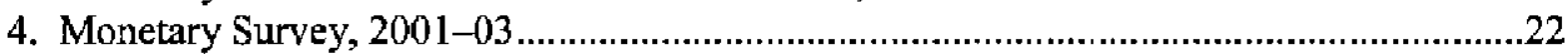

5. Implicit Subsidies and Losses in the Energy Sector, 2000-04 _...................................24

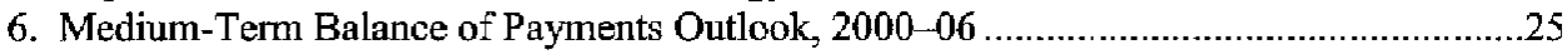

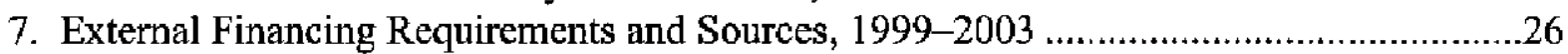

8. Indicators of External Vulnerability, 1997-2003 ...............................................27

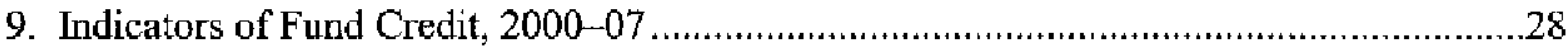

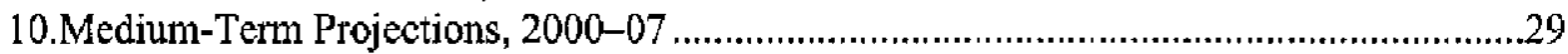

Appendices

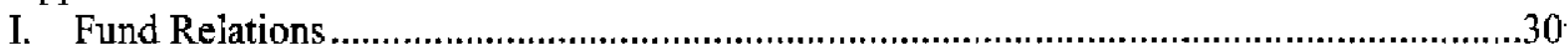

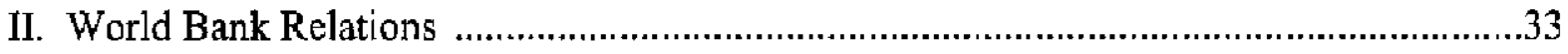

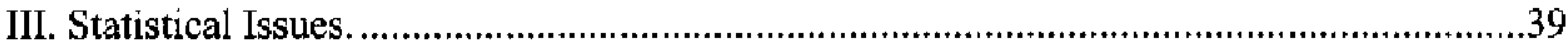

IV. Letter of Intent, Supplementary Memorandum on Economic and Financial Policies in 2003, and Technical Memorandum of Understanding 


\section{INTRODUCTION}

1. The 18-month Stand-By Arrangement (SBA) for Romania in the amount of SDR 300 million, or 29 percent of quota, was approved on October 31, 2001. Romania made one purchase of SDR 52 million upon approval and another of SDR 82.67 million upon the completion of the combined first and second reviews on August 28,2002. Discussions on the third review were held in Bucharest October 6-17, 2002 and February 11-19, 2003. ${ }^{1}$

\section{In concluding the last Article IV consultation in January 2003, Executive} Directors noted that accelerated structural reforms were required to sustain favorable macroeconomic developments. They emphasized that vigorously addressing the problem of losses and quasi-fiscal activities of state-owned enterprises (SOEs) would be crucial for completing Romania's transition to a fully operational market economy, and for paving the way for EU accession. In particular, they considered that further progress in enforcing hard budget constraints required stepping up the fight against the nonpayment of utility bills and tax arrears. Directors also urged the authorities to accelerate the closure of nonviable enterprises; continue to reduce employment in SOEs as a necessary condition for reducing losses and accelerating privatization; and to strengthen wage discipline in this sector.

\section{Romania's macroeconomic performance continued to be favorable, but} slippages in wage policy and privatization delayed the completion of the third review. Robust GDP growth continued throughout 2002, and progress in reducing inflation and the current account deficit was better than targeted. However, financial discipline in the stateowned sector remained weak and the end-September performance criterion on the wage bill in monitored SOEs was not observed. Although the performance criteria on the collections of the main utilities were met, the sustainability of these results is not ensured. In particular, the payment discipline of mines and SOEs scheduled for privatization weakened toward the end of 2002 and in early 2003. Privatization in the enterprise sector remained behind schedule. Regarding the privatization of the largest bank, $\mathrm{BCR}$, the second invitation for expressions of interest in November was unsuccessful (SM/02/381, $\mid 17)$ and the bank could not be sold to a strategic investor by February 2003 (structural PC).

\footnotetext{
I The mission teams included Messrs. Mates (head), Westphal, Gueorguiev, and Carnot (all EU1), Mr. Lall (PDR), Mr. Abiad (RES), Mr. Harjes (AFR), Ms. Rivera and Ms. Villar (staff assistants EU1). The Fund's resident representatives, Mr. Cosse, and his successor since January 15, Mr. Justice, assisted the mission. Discussions were held with Prime Minister Năstase, Governor of the National Bank of Romania (NBR) Isarescu, Minister of Public Finance Tanascscu, Minister of Labor Sârbu, Minister of Industry Popescu, Minister of Privatization Musetescu, and Minister for Development and Forecasting Cazan. Ms. Marinescu, Advisor to the Executive Director for Romania, participated in the meetings.
} 
4. To fully achieve the objectives of strengthening financial discipline, privatization and structural reforms during the arrangement, the authorities have requested an extension of the arrangement period by $5 \frac{1}{2}$ months until mid-October. They are also requesting waivers for the nonobservance of the end-December quantitative performance criterion on the SOE wage bill and the structural performance criterion on the privatization of $\mathrm{BCR}$, on the basis of measures presented in the Supplementary Memorandum on Economic and Financial Policies in 2003 (SMEFP-2, Appendix IV). It is also proposed that the purchases be rephased, with SDR 55.111 million made available following the completion of the third review, and SDR 110.223 million following the last, fourth, review. Staff considers the requests for waivers and the extension as warranted, in the context of the authorities" decision to proceed with intermediate steps toward the privatization of BCR, and with an ambitious restructuring program in 22 companies scheduled for privatisation.

5. The authorities' highest priority is progress toward EU accession, with the successful completion of the SBA seen as an important step in this direction. Public support for the government remains high after the EU accepted 2007 as the target date for Romania's accession. However, the government is facing resistance from several trade unions, which oppose the restructuring and downsizing of large loss-making SOEs. Parliamentary and presidential elections are scheduled for late 2004 or early 2005.

\section{Recent Economic Developments and Performance Under the Program}

\section{GDP growth was strong in 2002 , but industrial production somewhat weakened} at the turn of the year. Fiscal contraction, and even more the ambitious energy price adjustments, slowed growth in household consumption from 6 percent in 2001 to 3 percent in 2002 (Text Table 1). On the other hand, exports strengthened during most of 2002, as Romania's competitiveness more than offset the effects of the weak demand in partner countries (Figures 1 and 2). Private investment, in response to an improvement in the business climate and easing credit conditions increased by 12 percent. Exports, however, flattened toward the end of 2002 , in line with further weakening in the EU, and industrial

Figure 1. Industria] Production and Exports, 2000-03 1/

$(1999=100)$

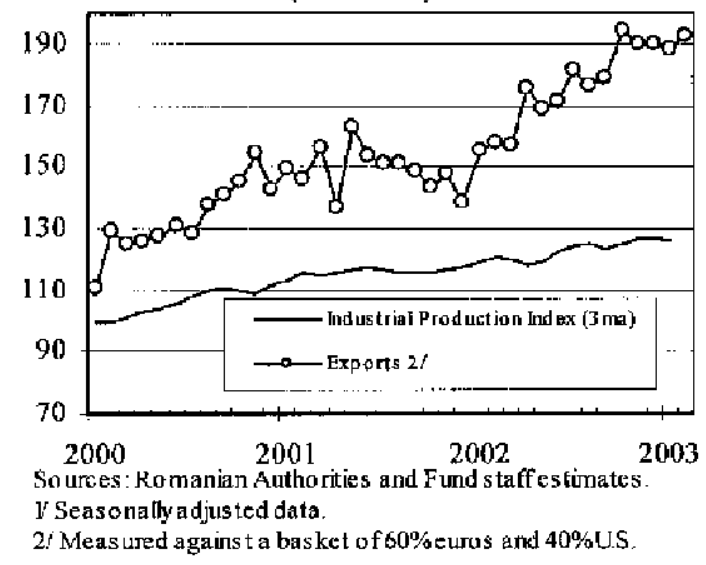

Figure 2. Trading Partners' Import Demand and Romania's Exports 2000-03 (percent change trom tour quarters earlıer)

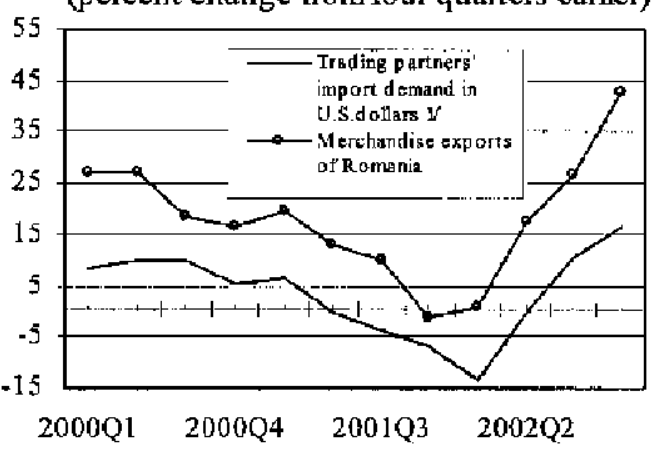

So urces : Romarian authorites, Drection of Trade Statis tics (DOTS) and Fund s taff esstimates. V Trade-weighted average for Rom ania"s trading parners. 2002 Q 104 are estimates. 
production fell slightly in December and January, increasing somewhat uncertainties about the outlook for 2003 .

Table 1. Ramania: Main Economic Indicators, 2001-04

(year-on-year growth rates, except where indicated, in percent)

\begin{tabular}{|c|c|c|c|c|c|c|c|c|}
\hline & \multicolumn{2}{|c|}{2001} & \multicolumn{3}{|c|}{2002} & \multirow[t]{2}{*}{2002} & \multirow{2}{*}{$\frac{20003}{\text { Proj. }}$} & \multirow{2}{*}{$\frac{2004}{P_{\text {Tij }}}$} \\
\hline & $\mathrm{HI}$ & $\mathrm{H} 2$ & Q1 & $\mathrm{Q} 2^{2}$ & Q3 & & & \\
\hline GDP (antual teal growth) & 4.7 & 5.5 & 3.1 & 5.7 & 4.4 & 4.9 & 4.9 & 5.0 \\
\hline Final domestic demand (ankual real growth) & 6.4 & 5.5 & 3.3 & 3.6 & 4.3 & 3.9 & 5.7 & 5.0 \\
\hline Total consumption (ampual recal growth) & 6.5 & 5.3 & 3.1 & 3.0 & 3.5 & 3.0 & 4.6 & 3.6 \\
\hline Gtoss fixcod capital formation (annual real growth) & 6.4 & 6.2 & 4.8 & 6.3 & 7.6 & 8.3 & 10.7 & 10.6 \\
\hline Net exports & -4.6 & -2.8 & I.I & 2.6 & -1.9 & 0.7 & -1.5 & -0.7 \\
\hline Fxports of goods and services & 20.8 & 6.3 & 3.8 & 30.6 & 18.0 & 16.8 & 11.2 & 7.5 \\
\hline Imports of goods and services & 29.7 & 12.8 & 1.1 & 2.6 & 23.0 & 12.1 & 12.2 & 7.2 \\
\hline Curepn accuun (in perscat of annual (ODP) & -3.4 & -2.5 & 0.5 & .1 .5 & -0.6 & -3.4 & -4.5 & -4.5 \\
\hline Anmual Inflation (cop) & 35.7 & 30.3 & 25.1 & 24.0 & 19.8 & 17.8 & ] 4.0 & 10.0 \\
\hline Otficial rescryes (eop, US3 billion) & 4.6 & 4.9 & 5.1 & 5.7 & 6.1 & 7,0 & 8.0 & 8.8 \\
\hline
\end{tabular}

Sources: National Irstitute for Statistics; Ministry for Econowic Development and Forecasting; and Fund staff projections.

\section{Inflation declined faster than projected, from 30 percent at end-2001 to}

18 percent at end-2002 (Figure 3). With labor costs in 2002 increasing by less than 1 percent in real terms and depreciation slowing to 15 percent relative to the basket, cost pressures eased, while slower consumption growth moderated demand. In early February, the headline inflation rate fell to 16 percent, the lowest level since the onset of transition.

However, after 24 months of continuous decline, the rate will pick up in March, partly owing to the low 2002 base. Core inflation, which over the last three months has averaged about 1.1 percent per month, will likely increase until cost and demand pressures from faster wage growth have abated (Figure 4).

Figure 3. Consumer Price Index $(\mathrm{CP})$ and Wages, 2000-03

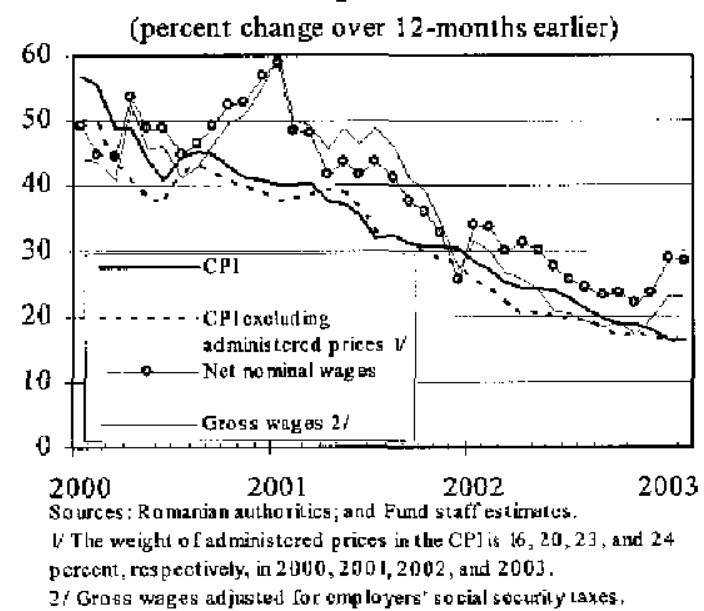

Figure 4. CPI Excluding Administered Prices, 2000-03 (percent change over earlier month)

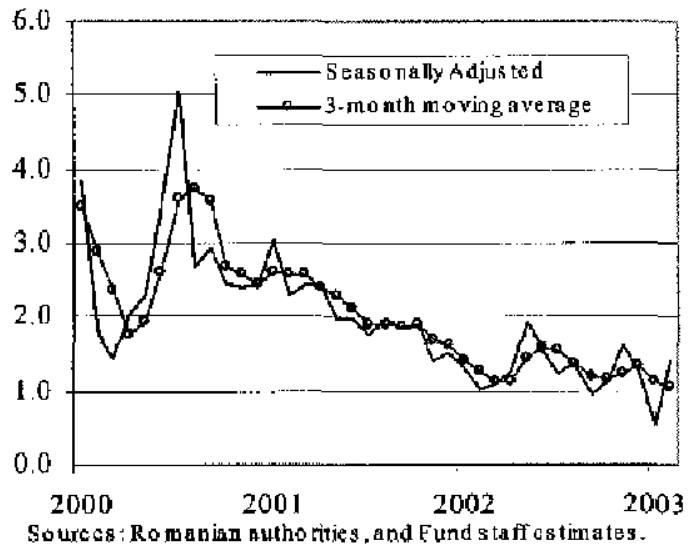


8. External competitiveness further improved in 2002. Owing to the moderate growth in wages and rising productivity, the real effective exchange rate in ULC terms depreciated by $6 \frac{1}{2}$ percent in 2002 (Figure 5). The REER in CPI terms, adjusted for administered prices, appreciated by a modest 0.9 percent. Romania also gained market share in its main export markets (Figure 2), while wage share indicators suggest that the profitability of Romania's producers has improved (Figure 6).

Figure 5. Real Effective Exchange Rate, 1996-2003

(3-Month Moving Average)

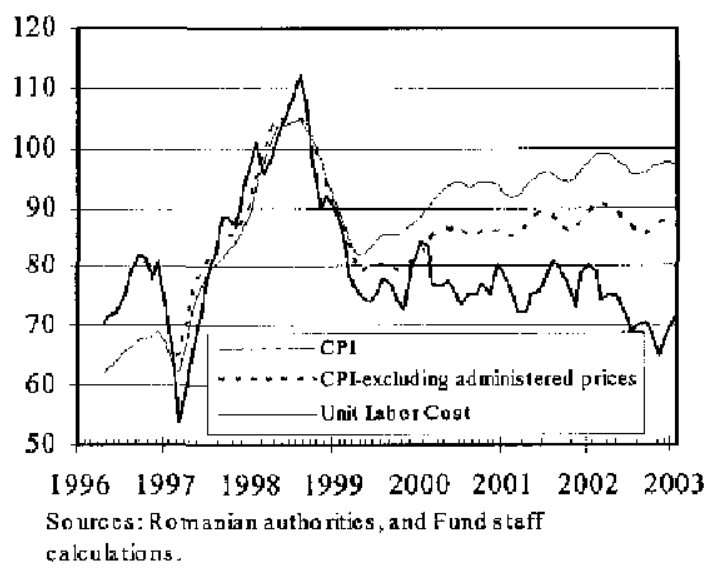

Figure 6. Unit Labor Cost Deflated by Producer Price Index, 1996-2003

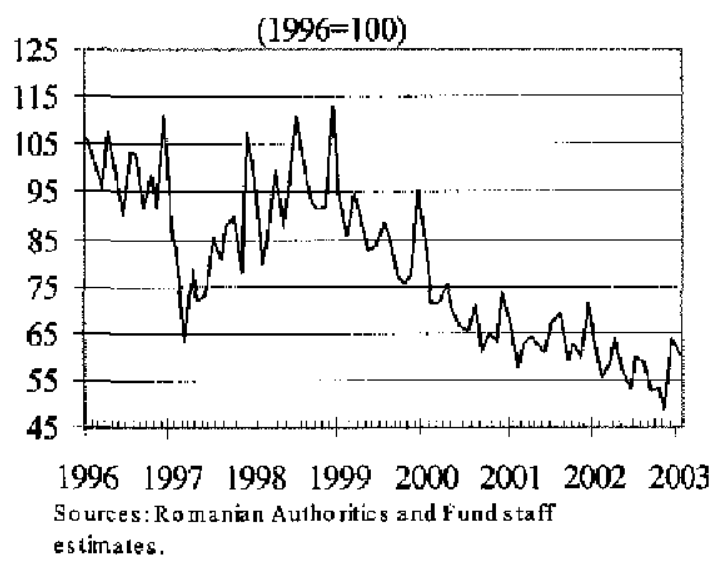

9. The external position strengthened more than expected. In addition to the improved trade balance, an increase in unrequited transfers, only partly reflecting better measurement, reduced the current account deficit to a surprisingly low 3.4 percent of GDP in 2002. Official reserves increased by US\$1.9 billion, to a comfortable level of four months of prospective imports. Market sentiment toward Romania has continued to improve and the sovereign bond spreads significantly narrowed between mid-2002 and February 2003. A modest widening in March, shared with other countries in the region, reflected a portfolio shift from European and Asian emerging market bonds to sub-investment grade Latin American bonds (Figure 7). Romania's sovereign foreign currency risk was upgraded by Standard \& Poors from B+ to BB- on February 27, 2003. 
Figure 7. Reserves, Exchange Rates, and Bond Spreads, 2000-03

\section{Rescryes}

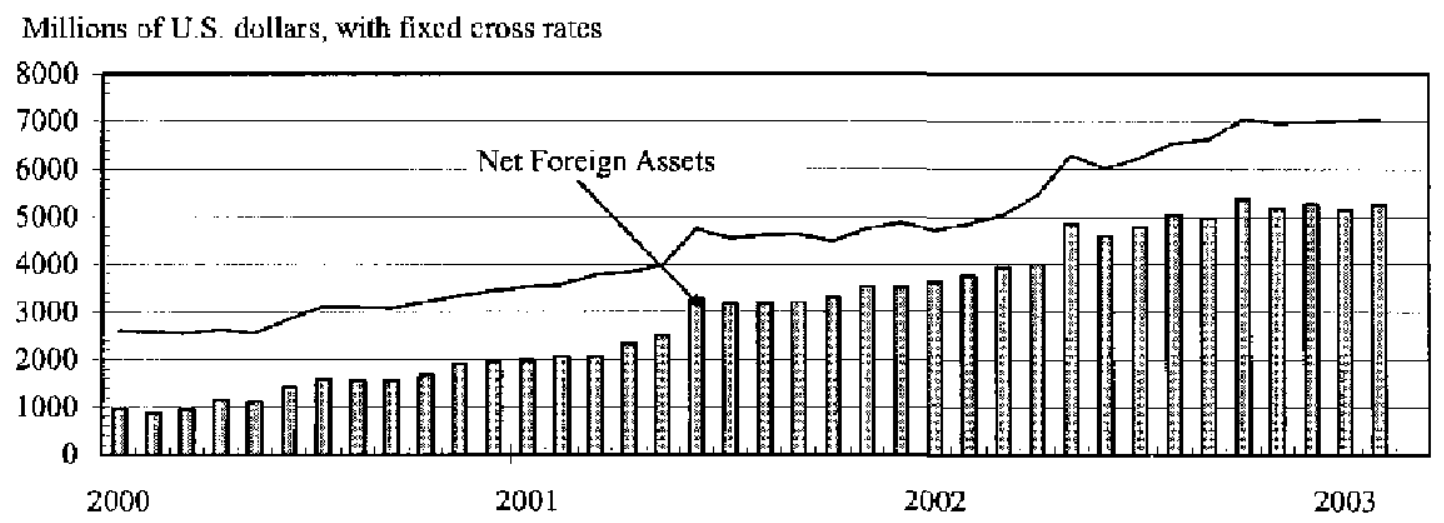

Monthly Depreciation Rate of Lei,

Three-month Moving Average

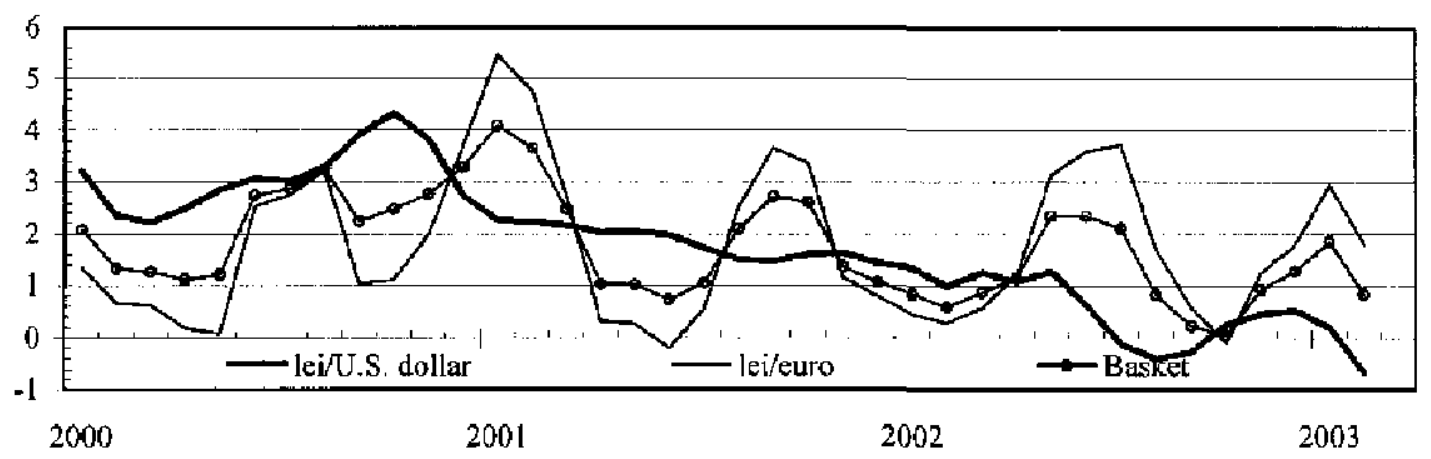

Bond Spreads

Basis points

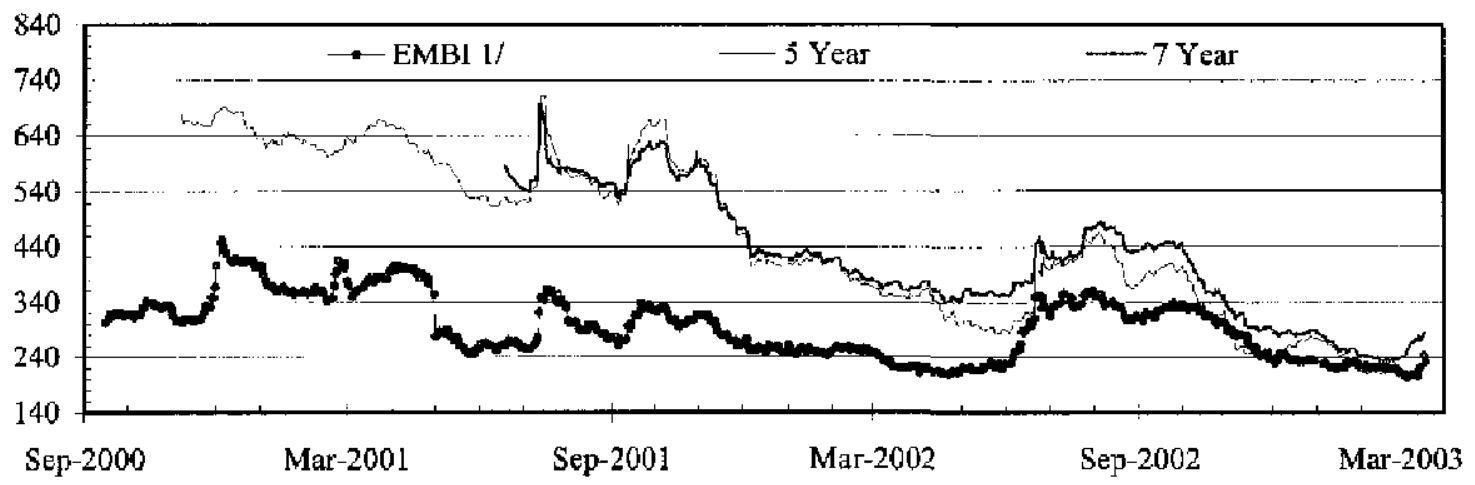

Sources: Romanian authorities; Fund staff calculations and Bloomberg.

1/ Euro EMBI Global index excluding Latin America. 
10. Budgetary consolidation in $\mathbf{2 0 0 2}$ was larger than targeted, but revenue collection underperformed. The deficit of the general government fell to 2.6 percent of GDP, compared with the original target of 3 percent of GDP (Text Table 2). Revenue collections were short of the revised target by about $1 / 4$ percentage point of GDP, as effects of measures to improve tax administration were delayed, but the shortfall was more than offset by lower interest payments and savings in matemity leave benefits.

Table 2. Hudget Deficit and Quasi-Fiscal Easses in the Energy Sector (In percent of GDP)

\begin{tabular}{|c|c|c|c|c|c|c|c|c|c|}
\hline & 1998 & 1999 & 2000 & 2001 & 2002 & & 2002 & 2003 & 2004 \\
\hline & Actual & Actual & Actual & Actual & Program & & Est. & Prog./Proj. & Proj. \\
\hline Deficit of the General Consolidated Government & 5.4 & 3.6 & 4.0 & 32 & 29 & $1 /$ & 2.6 & 2.7 & 2.5 \\
\hline Total Implicit Subsidies and Losses in the Eatrgy Stctor & $\ldots$ & $\ldots$ & 4.7 & 4.8 & 2.7 & & 2.5 & 2.0 & 1.4 \\
\hline
\end{tabular}

I/ Reflects an upward revision in noninal GDP. The original target was 3 percent of GDP.

Sources: Minssry of Public Finance, ANRE (electricity regulatory agency), Termoelectrica and Distrigaz Nord and Sud;; Work Bank; and Fund staff estimates.

\section{The $\mathbf{2 0 0 3}$ budget is in line with understandings reached in the second review} under the program. The deficit target was set at 2.65 percent of the GDP estimate at that time, about $1 / 4$ percentage point below the 2002 target. Revenue, which was cautiously projected and assumed some underperformance in the 2002 collections, will benefit from the full-year effect of VAT and profit tax reforms enacted in mid-2002 and the broadening of the social security tax base. This will allow for a 5 percentage points cut in social security tax rates (from 57 percent to 52 percent of gross wages, with the reduction almost equally split between employers and employees), and increased capital spending (SMEFP-2, $\uparrow 10$ ).

\section{Losses in the energy sector declined in 2002 relative to GDP by $2 \frac{1}{2}$ percentage} points, reflecting energy price increases and improved collections in the gas sector. However, households' arrears to district heating companies increased, which the authorities attributed to the sharp increase in heating prices. While the collection targets for end-2002 were observed, payment discipline of several large state-owned companies deteriorated in late 2002 and early 2003 , putting these achievements at risk.

\section{After slippages relative to program} targets in Q1-Q3, the authorities succeeded in moderating wage growth in monitored SOEs in Q4 2002 (Figure 8). This was achieved by cutting or postponing bonus payments in virtually all monitored companies. As a result, the average annual wage growth in these SOEs, after overshooting private sector wages by a large margin in Q1-Q3 of 2002, was successfully brought down in Q4. As a result, for the whole year, SOEs wages increased in line with those in the private sector, by
Figure 8. Real Wage Growth, 2000-03 // 2/

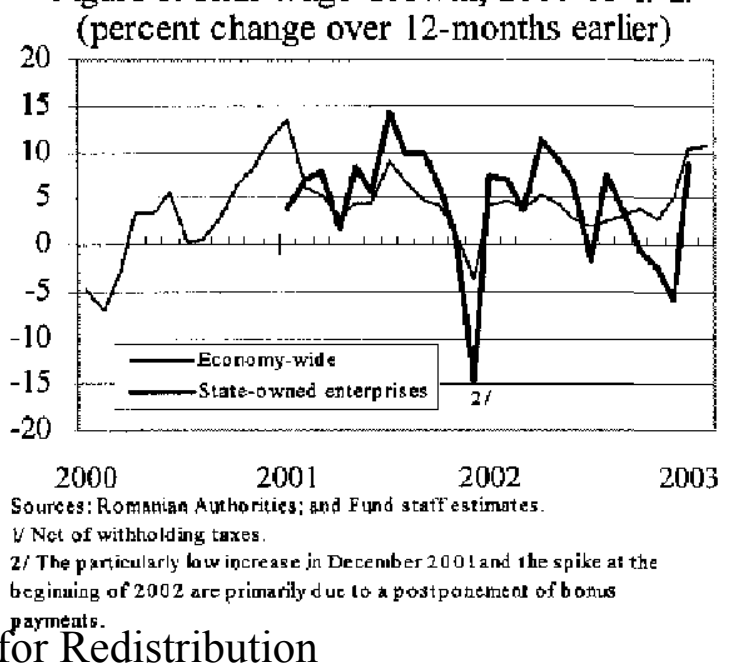


about $3 \frac{3 / 4}{4}$ percent in real terms. However, one railway company used a loophole in meeting its target and, as a result, the revised target for the wage bill at end-December was exceeded, albeit by a negligible 0.2 percent. On balance, the implementation of wage policy has been tightened since the completion of the first and second reviews. The 2003 SOEs wage program, approved in December, has limited their nominal wage bill growth to 14 percent. This would imply moderate net real wage growth of about $3 \frac{1}{2}$ percent.

\section{Faster-than-expected disinflation and} robust demand for lei-denominated assets allowed for further interest rate cuts, while the NBR continued with purchases of foreign currency to keep the exchange rate in line with its inflation target. Since end-August 2002, the NBR has progressively cut its policy interest rate by 8 percentage points, thereby reducing the real interest rate to 5 percent on a 12 -month forward-looking basis (Figure 9). The interest rate cuts and a measure to liberalize outward capital flows reduced the surplus in the foreign exchange market, with the NBR purchasing about US $\$ 130$ million between January 1 and mid-March, about a half of the amount in the same period of 2002 .

\section{The process of rapid remonetization of} the economy has continued. With disinflation on track and foreign reserves steadily rising, confidence improved and the demand for lei-denominated assets (time deposits and T-bills) has strengthened. As a result, growth in lei broad money rose to about 26 percent in real terms in January. The T-bill yield curve has continued to shift downward, and has stayed inverted for almost two years, indicating that expectations for further disinflation remain strong (Figure 10).

Figure 9. Evolution of Nominal Interest

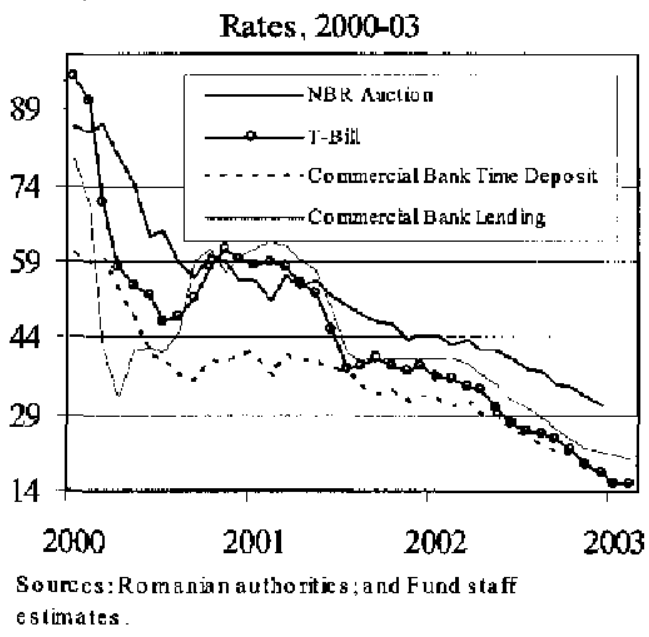

Figure 10.Treas ury Bill Yield Curve, 2002-03

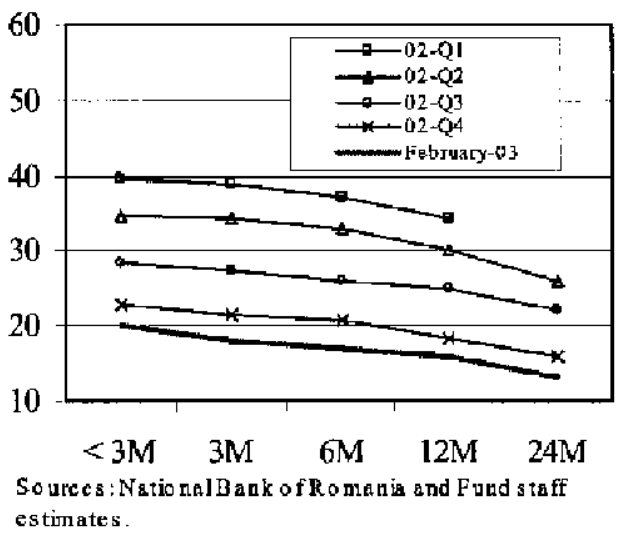

16. Bank credit to the nongovernment increased rapidly throughout 2002 . Yearly credit growth, of about 33 percent in real terms as of December 2002, is dominated by foreign currency denominated lending, as borrowers appear to believe that the risk of exchange rate depreciation does not fully match the interest differential. Moreover, mandatory reserve requirements imposed bigher spreads between lei denominated deposits and loans than on those in foreign currencies. To alleviate this effect, the NBR reduced the reserve requirement for lei deposits and raised it for foreign currency deposits in November. Credit to the state-owned sector increased at the end of the year on account of publicly- 
guaranteed credits for investments in hospitals and in the communication sector, as well as higher domestic than foreign borrowing by the energy sector.

17. The new banking regulation provides for more prudent loan classification and provisioning. Effective January 1, it requires that the financial performance of the borrower, in addition to their payment history, be taken into account in the classification and provisioning of loans, thus better reflecting repayment prospects. The provisions were increased by a modest 40 percent.

18. Structural benchmarks for privatization have been only partly met and progress in the energy sector has been very slow. Six instead of the targeted eight large companies were privatized in Q4 of 2002. In the energy sector, some preliminary steps have been accomplished, though with substantial delays. The Ministry of Industry accepted the World Bank's request for transparent tender procedures only after a protracted discussion. The tenders for privatizing the two electricity distribution companies were launched on January 8 , and have attracted the interest of five investors. The contracts with privatization advisors for the oil company Petrom, the largest firm in the country, and the gas distribution companies were signed, after delays, in mid-December 2002 and in early March 2003, respectively.

\section{POLICY Discussions}

19. Discussions on the $\mathbf{2 0 0 3}$ macroeconomic framework took place in the context of a better-than-targeted performance in the previous year but also a weaker external environment. Owing to the importance of the minimum wage increase for macroeconomic developments in 2003, discussions focused on the assessment of its effects on domestic demand, the current account balance, and inflation. The implementation of the 2003 wage program for SOEs and the enforcement of financial discipline in the state-owned sector were seen as the main instruments to contain economy-wide wage pressures.

20. Staff cautioned, and the authorities agreed, that Romania needed to build on the successful macro-stabilization of the last two years, and press ahead with structural reform to underpin these favorable developments. Staff emphasized that with the uncertain prospects for the global economy and Romania's still incomplete transition to a fully-functioning market economy, there was no room for complacency and called for perseverance in implementing structural reforms. The authorities reaffirmed their commitment to the longstanding issue of restructuring large loss-making SOEs with a view to facilitate their privatization. They also decided to start negotiations with the IFC and EBRD on a transitory investment in BCR. Staff welcomed both decisions.

\section{A. Macroeconomic Framework for $\mathbf{2 0 0 3}$}

21. The large minimum wage increase of 43 percent, which was decided in June 2002 and became effective in January 2003 , will be a key determinant of macroeconomic developments. Even with the reduction in employees' social security contributions, this pushed the year-on-year growth in net economy-wide wages to 29 percent 
in nominal, and about $10 \mathrm{l} / 2$ percent in real terms in January and February (Figure 8). While this one-time increase will be gradually eroded by inflation, the size of feed-through effects remains uncertain. Crucial in this respect will be the forthcoming collective contracts comprising state-owned and private enterprises, which in the past have often used the minimum wage as a benchmark. A limited set of such contracts concluded in January, suggests that wages could grow on average by $25 \%$ percent in nominal terms in 2003 , or 8 percent in real terms based on projected CPI inflation, close to the staff estimate at the time of the second review. Real labor costs, benefiting from a reduction in payroll taxes, would grow by about 3 percentage points less.

22. With the recent overperformance on the current account and inflation providing a cushion, staff concurred that the policies for 2003 agreed under the second review remained appropriate but stressed the importance of consistent implementation. Assuming that the wage growth is contained as suggested by the first round of collective contracts, growth in labor cost would be broadly in line with productivity and projected inflation. Therefore, the authorities' target to reduce inflation to 14 percent by end-2003, which became less ambitious owing to the overperformance in 2002 , would remain within reach. From the demand side, the strengthening in consumption would lead to a widening in the current account deficit in 2003 , to about $4 \frac{1}{2}$ percent of GDP, taking account also of a somewhat weaker external environment and energy price shocks. This level would still be below the original 2003 progran target. GDP growth of about 5 percent could be achieved assuming at least some recovery in the EU, and a strengthening in investment on the back of a forceful implementation of structural reforms and privatization.

\section{Macroeconomic tightening will be required should additional demand pressures} develop. Staff warned that this macroeconomic framework could be put at risk if wages grow faster than projected. In particular, staff reiterated that the authorities should remain vigilant and ensure a strict implementation of the SOE wage program. This was seen as crucial for ensuring that the collective contracts yet to be signed, particularly in the sectors where presence of the state-owned sector is important, do not lead to higher wage growth than projected. The authorities remained confident that both SOEs and private sector wages would grow less than estimated by staff, and argued that a substantial portion of the reported wage increase in January reflected legalization of previously nonreported payments, as suggested by the faster wage growth in small companies. However, they confirmed their commitment to tighten fiscal policy later in the year if wage developments put the current account and the disinflation targets at risk.

\section{B. Fiscal Policy}

24. Discussions on the $\mathbf{2 0 0 3}$ budget focused on the need to eliminate risks to revenue and to provide additional resources for targeted heating subsidies and enterprise restructuring. The revenue side of the 2003 budget was affected by a cut in specific oil excises on February 1, and an Emergency Ordinance (EO) which envisaged that the utilities could offset arrears of 23 SOEs by reducing their tax and other payments to the budget (SMEFP-2 ף19). To restore the integrity of the budget, the authorities undertook to increase 
the fuel excises back to their old level on May 1. They also eliminated the offset scheme for utilities. Reflecting these measures and the beneficial impact of euro appreciation on revenue from euro-denominated oil excises, the estimated shortfall of revenues compared to the budget was reduced to 0.1 percent of GDP. On the expenditure side, the authorities decided to double heating support for low-income families and cancel some of their arrears. Staff supported the first measure, but noted that the second one would not only adversely affect the financial performance of heating companies, but would also undermine customers' incentives to pay in the future. In response, instead of canceling households' arrears, the authorities decided to use one-time assistance from the budget to pay for the arrears of the very poorest group of households. An emergency ordinance, approved in March, has also provided resources for mitigating the social impact of the restructuring of 22 large companies (SMEFP-2, $\uparrow 12$ ). These measures will be financed through savings in interest expenditure, reserves in the unemployment fund budget, and tightening eligibility criteria for generous maternity leave benefits, applications for which recently strongly increased. The increase in off-budget guarantees to US\$650 million reflects mainly investments in railways and the Cernavoda nuclear power plant, which are both expected to generate resources for repayment and will not burden the budget.

\section{Progress has been achieved in implementing the tax administration reform. As} envisaged, a separate tax administration department within the Ministry of Finance has been created and the responsible Secretary of State was appointed in January (SMEFP-2, q13). A large taxpayer directorate for Bucharest has been established, and preparations for establishing a unified administration for the collection audit and enforcement of social security contributions under the Ministry of Finance are broadly on track.

\section{Staff strongly recommended that the authorities reject the introduction of a} reduced VAT rate under discussion for 2004. The authorities indicated their intention to introduce in 2004 a reduced VAT rate for pharmaceuticals and basic food products, reflecting social considerations. Staff responded that this step was likely to undermine substantially the effectiveness of tax administration, which would have to focus on VAT refunds in a much larger number of cases. Moreover, staff emphasized that well-targeted transfers to lowincome households were superior to the possible social effects of a reduced VAT rate.

\section{Wage Policy in SOEs}

27. Staff reiterated the crucial importance of strengthening wage discipline in SOEs, the absence of which has often derailed stabilization policies in the past. While regretting that not a single PC on SOEs wage bill under the program had been met, staff acknowledged that the measures implemented in Q4 succeeded in reducing the cumulative average wage growth of SOEs in 2002 to the economy-wide average. Staff also noted the improvement in wage discipline in companies under the Ministry of Industry, where in the past collusion between management and trade unions had often hampered the implementation of wage policy. However, staff expressed concern about indiscipline in enterprises under the Ministry of Transportation. The authorities pointed out that the employee transfer procedure used by the railway company was banned by the new Labor Code. Looking forward, they argued that 
the timely approval of the 2003 budgets of the monitored SOEs would effectively constrain wage settlements, particularly in sectors where presence of SOEs was important. To strengthen the credibility of the 2003 wage bill program, they undertook to implement further employment reduction of 8,000 positions and a hiring freeze in most monitored SOEs (SMEFP-2, ๆ16). On balance, as the implementation of wage policy has been significantly tightened, staff supports the authorities' request for a waiver of the end-December PC.

\section{Privatization}

\section{The authorities acknowledged that reducing employment in SOEs was a} necessary condition for accelerating privatization. Until recently, progress in preparing large lossmaking SOEs for privatization has been slow, largely owing to collusion between management and trade unions. Against this background, staff considered the restructuring plan for 22 large lossmaking companies and several mines, which involves laying off at least 24,000 employees, to be an important step (SMEFP-2, $\{24$ and $\mid 26$ ). Staff considered the proposed severance payments, financed by the budget, as high relative to those used in other transition economies, but the authorities argued that it was essential to successfully accomplish the proposed retrenchment. While the layoffs, which are scheduled to be implemented in two waves, may not be sufficient to ensure the privatization of all concerned companies, they significantly improve the prospects.

29. The authorities have also decided to accelerate the closure of some unviable SOEs, but staff suggested a more vigorous approach. Acknowledging that keeping insolvent companies afloat is more expensive than improving the conditions for the creation of new employment, the authorities decided to start liquidation procedures for a number of SOEs, unless privatized by mid-year (SMEFP-2, q25). While welcoming this decision, staff urged the authorities to accelerate the closure of a larger number of companies that were highly unlikely to be privatized. Staff also advised the authorities to review bankruptcy procedures, which remained highly inefficient, non-transparent and slow.

30. To prepare BCR for eventual sale to a strategic investor, the authorities decided to sell a minority share to the EBRD and the IFC. The completion of the sale of 25 percent plus two shares (end-July structural performance criterion) will reduce the government's share in the bank to less than 50 percent (about 30 percent is owned by local investment funds), and give the two IFIs a blocking minority position. A shareholding agreement is also expected to provide for representation of the two institutions in the supervisory and auditing boards. To complete this transaction on time, the authorities have provided the two IFIs access to requested data and are committed to ensure that all other legislative and administrative preconditions are implemented on time (SMEFP-2, $\mid 26$ ).

\section{E. Financial Discipline and Energy Sector Reforms}

31. Payment discipline among large energy users has remained problematic. Staff noted with concern that the plan for disconnecting industrial nonpayers had not been implemented. The authorities underscored the high social costs of effectively shutting down 
simultaneously several large companies, most of which are situated in disadvantaged areas, and the adverse effect of such a measure on possible privatization. They noted, however, that the envisaged labor retrenchment in large industrial lossmakers will improve their financial performance. Until these measures produce results, the government and the staff agreed that, instead of tolerating arrears to utilities, the budget would pay utility bills for selected companies until end-June (SMEFP-2, \$18). Having in this way reduced the risk of simultaneous closure of several large enterprises, the authorities decided to move aggressively with disconnections against nonpayers (SMEFP-2, ๆ18).

\section{The authorities reiterated their commitment to continue energy price}

adjustments. The producer price for thermo-electric power will be maintained at US $\$ 39$ per Mwh, a level consistent with cost recovery even in the context of higher fuel prices in 2003. A two step adjustment of gas prices by a cumulative 13 percent in March and July will match the WEO projections for the expected increase in import prices in 2003 . The energy sector's losses would be reduced by $1 / 2$ percentage point of GDP. As a result of higher world fuel and gas prices, this reduction will be lower than ariginally anticipated ( 0.9 percentage points of GDP), but this would be compensated in 2004 as imported fuel prices decline.

33. Privatization in the energy sector is proceeding with delays. Following a three months delay, the privatization strategy for Petrom, the largest company in Romania, is now expected to be approved in May, and the tender for expressions of interest will be launched by end-June (SMEFP-2, [21). Offers for acquiring a majority holding of at least 51 percent for the two electricity distribution companies will be invited by mid-May, and the announcement of the privatization tenders for the two gas distributions companies is scheduled for June 30 .

\section{F. Monetary Policy and Banking Supervision}

\section{The NBR will continue to follow its gradual disinflation strategy, which has} delivered positive results. The Bank management considered that the inflation target of 14 percent for end-2003, set cautiously to reflect the uncertainties about wage developments, was easily within reach. The NBR did not see harmful effects from the minimum wage increase yet, as inflation continued its rapid decline in January and early February. They also concurred with the argument on legalization of unreported payments. On the exchange rate, the NBR will continue with purchases in the foreign exchange market with the aim of guiding the exchange rate on a path consistent with the disinflation target. A moderate CPIbased real effective appreciation was considered consistent with preserving competitiveness, with the Balassa-Samuelson effect estimate of 2 percent serving as a broad reference in this respect. The NBR will support disinflation by keeping the deposit interest rates positive in real terms.

\section{Staff noted that the scope for further interest rate cuts would depend on} progress in reducing inflation. Following recent cuts, to 20 percent the main policy interest rate is no longer excessively high, as evidenced by the diminishing excess supply in the foreign exchange market. Staff reiterated that, despite the recent overperformance in 
reducing inflation, a possible downward revision in the 2003 target contemplated by the NBR should not be considered until developments in wages and domestic demand can be assessed with more certainty.

36. Staff reiterated concerns about the rapid expansion of foreign-currencydenominated credit. The NBR considered that the real credit growth was desirable in the context of the low degree of financial intermediation, and that the bias in favor of foreigncurrency-denominated lending would be reduced by the recent differentiation in reserve requirements and declining domestic interest rates. Furthermore, the enhanced supervision over the banks with the fastest credit expansion did not uncover any substantial problems in their credit portfolios. Staff cautioned that over the medium-term, such problems could be triggered by an unexpected currency depreciation, when borrowers' exchange rate risk could be transformed into banks' credit risk. Against this background, staff reiterated the recommendation to consider imposing higher capital requirements against lending in foreign exchange, if the current very strong growth continues.

\section{MEDIUM-TERM OUTlOOK, EXTERNAL VulNERABILITY, AND CAPACITY TO RePAY THE FUND}

37. The medium-term outlook has remained broadly unchanged since the conclusion of the Article IV consultation in January 2003, and Romania's external vulnerability remains relatively low in the short term. Official reserves are now at a comfortable level in terms of import coverage and broad money, and currently exceed by a large margin shortterm debt by remaining maturity (Table 8 ). The overperformance in reducing the current account deficit in 2002 created a cushion for a widening in 2003 triggered by the minimum wage increase and higher international fuel prices. As the less favorable economic outlook for Romania's major trading partners has had modest impact on exports so far, the lower export growth rate projected for 2003 , compared with 2002 , appears to be within reach, even if the expected recovery in Europe does not materialize. While capital inflows and market sentiment are expected to remain favorable in the medium-term, a decline in appetite for risk and geopolitical uncertainty, which are already affecting emerging market bond spreads, present a downside risk for the projected 2003 inflows. The remaining purchases under the SBA are considered adequate to address these risks.

38. Romania's capacity to repay the Fund remains good. Over the medium term, debt service to the Fund, including that on prospective purchases would be moderate, averaging 0.6 percent of exports of goods and nonfactor services (Table 9). The medium-term public debt-service profile is fairly smooth, except for a few quarterly peaks due to maturing bond obligations.

\section{Program Monitoring}

39. The current arrangement is proposed to be extended by $51 / 2$ months, to mid-October 2003. The last review will be based on an end-June test date, and the 
completion of the sale of the minority share of BCR to the EBRD and the IFC by end-July 2003. Quantitative performance criteria are proposed for end-June 2003, and structural performance criteria and benchmarks through end-July 2003. Several structural benchmarks have been added to the program, which aim at strengthening privatization efforts and enforcing a hard budget constraint for SOEs. To improve collections in the energy sector, the indicative target for the main electricity distributor has been converted into a PC for end-June 2003. Prior actions, structural performance criteria and structural benchmarks, which

comprise corrective measures for slippages under the program, are specified in Table 2 of the SMEFP-2.

\section{Staff Appraisal}

\section{In 2002, the Romanian economy continued to benefit from the strong}

stabilization policies of the past few years. On the policy front, the authorities persisted in their efforts to curb quasi-fiscal deficits in the energy sector, most recently by implementing unpopular but necessary price adjustments. Together with continued budgetary restraint, this led to a slowing of consumption growth, a strengthened current account, and a further deceleration of inflation while output growth has remained high. In tandem with some progress on structural reforms, consumption growth moderated and exports and investment became the main engines of growth. As a result, the economy weathered the global economic slowdown well, as indicated by strong GDP growth for the second year in a row.

41. The issue confronting the authorities is how to sustain these welcome, if classic, outcomes of policies of stabilization and reform. Experience elsewhere has too often been that the realization of the improved outcomes gives way to a period of more mixed performance as the drive for discipline and reform flags and structural inefficiencies assert their influence. To their credit, the authorities intend to persevere. Given the uncertainties emanating from the global economic environment and Romania's unfinished structural reform agenda, there is no room for complacency. This seems to be all the more critical at this juncture in view of the high risks created by the minimum wage hike. Against this background, it is essential that the authorities implement the agreed policies fully, notably on SOE wages, the pace of privatization and structural reforms. Perseverance in reform efforts will be particularly important after the expiration of the current Stand-By Arrangement in the run up to the 2004 elections.

42. Full implementation of wage policy will be crucial. Given the repeated nonobservance of the relevant performance criteria, the decisive implementation of SOEs wage program will be the subject of particular attention in the fourth review. Moreover, if the government does not succeed in containing the spill-over effects of the minimum wage hike and stronger wage pressures develop than suggested by the recently signed collective contracts, in either the public or private sector, it will have no option but to tighten fiscal and credit policies.

43. Budget expenditure in 2003 will need to be reduced swiftly if fiscal policy needs to be tightened or weaker-than-expected economic growth negatively affects revenue. 
Cuts should preferably be in subsidies and insufficiently targeted social transfers. To prepare for these contingencies, the authorities would be well advised to accelerate ongoing work to prioritize expenditure programs.

44. Having successfully completed the comprehensive VAT and profit tax reform, the authorities should avoid eroding its benefits. Fiscal policy remains constrained by the need to further reduce payroll taxes, which contimue to be among the highest in all EU accession countries, and the need to provide resources to meet EU accession-related requirements. The introduction of a reduced VAT rate, being contemplated by the authorities, would undermine the effectiveness of the tax administration while achieving little benefit in terms of social fairness.

45. The resolve in implementing the tax administration reform will need to be sustained. Progress in implementing the tax administration reform has been commendable. As a next step, it will be important to ensure that administrations for the collection of social security contributions are unified by January 2004. Subsequently, the collections of social security taxes should be integrated with the administration of income tax.

46. The government has succeeded in reducing energy sector losses, but further efforts to enforce hard budget constraints are required. In this context, the vigorous implementation of the authorities' plan to enforce payments and disconnect nonpayers is crucial, as is determined progress in the privatization of energy distribution companies. The increased social assistance to low-income households should also help improve collections in the heating sector. Energy sector privatization will be crucial for fully enforcing discipline in payments to utilities, given the continued prevalence of weak corporate governance in the Romanian state-owned sector.

47. Slow progress in structural reforms continues to expose macroeconomic stability to risks, but the authorities' plan for downsizing large lossmaking SOEs is a major change in attitude. Largely reflecting the practice of imposing conditions on investors to preserve employment and commit to investment, progress in privatization has been slow. The decision to substantially reduce employment in 22 large lossmaking SOEs is therefore an important and promising change. Adherence to the timetable for the restructuring plan will be crucial for establishing credibility in this area. It is also encouraging that the authorities intend to offer for sale most of the SOEs outside the energy sector and Petron, the largest company in Romania, by end-2003. However, the successful completion of privatization will require further downsizing in other companies. The authorities will also have to adopt a significantly more vigorous approach to liquidating unviable companies. In this context, the legislative and institutional framework for bankruptcy procedures, an area where Romania lags behind other accession countries, needs to be strengthened.

48. Strict adherence to the timetable for selling a minority share in BCR to the EBRD and IFC will also be of critical importance for completing the fourth review. As the two tenders for the sale of a majority share in BCR proved unsuccessful in the current business environment, staff supports the government's decision to sell a substantial share to 
the EBRD and the IFC with a view to prepare the bank for final privatization as soon as market conditions improve.

49. The monetary policy framework remains appropriate. The current policy of guiding the exchange rate consistent with the inflation target has been successful, and remains appropriate for the near future. While the recent sizable cuts in the policy interest rate were warranted in light of the success in disinflation and strong demand for lei assets, its current level is no longer excessively high. The scope for further interest rate cuts depends on supply pressures in the foreign exchange market and progress in reducing inflation, the latter heavily dependent on the success in containing the spillover effects of the minimum wage hike.

50. The rapid expansion of foreign-currency-denominated credit remains of concern as it exposes banks to higher credit risk. The FSAP already underway will provide an excellent opportunity for an in-depth look in vulnerabilities associated with this expansion. If the effects of the recently introduced measures to address this issue prove insufficient, the authorities should consider imposing higher capital requirements against lending in foreign exchange. The NBR should also vigorously enforce the implementation of the new regulation on loan classification and provisioning.

51. The staff considers that the authorities' revised program and their request for an extension deserve support. The authorities have reconfirmed their commitment to the program through the adoption of corrective mcasures, many of which have already been implemented. Thus, wage growth in the monitored SOEs has already been brought down to the economy-wide average in Q4 2002 and January 2003. The sale of a package of BCR shares to the EBRD and the IFC, in preparation for the eventual sale to a strategic investor, is moving forward on schedule. The staff therefore recommends the approval of the waivers, the completion of the third review, and the extension of the program through mid-October 2003. 
Table 1. Romania: Main Economic Indicators, 1999-2004

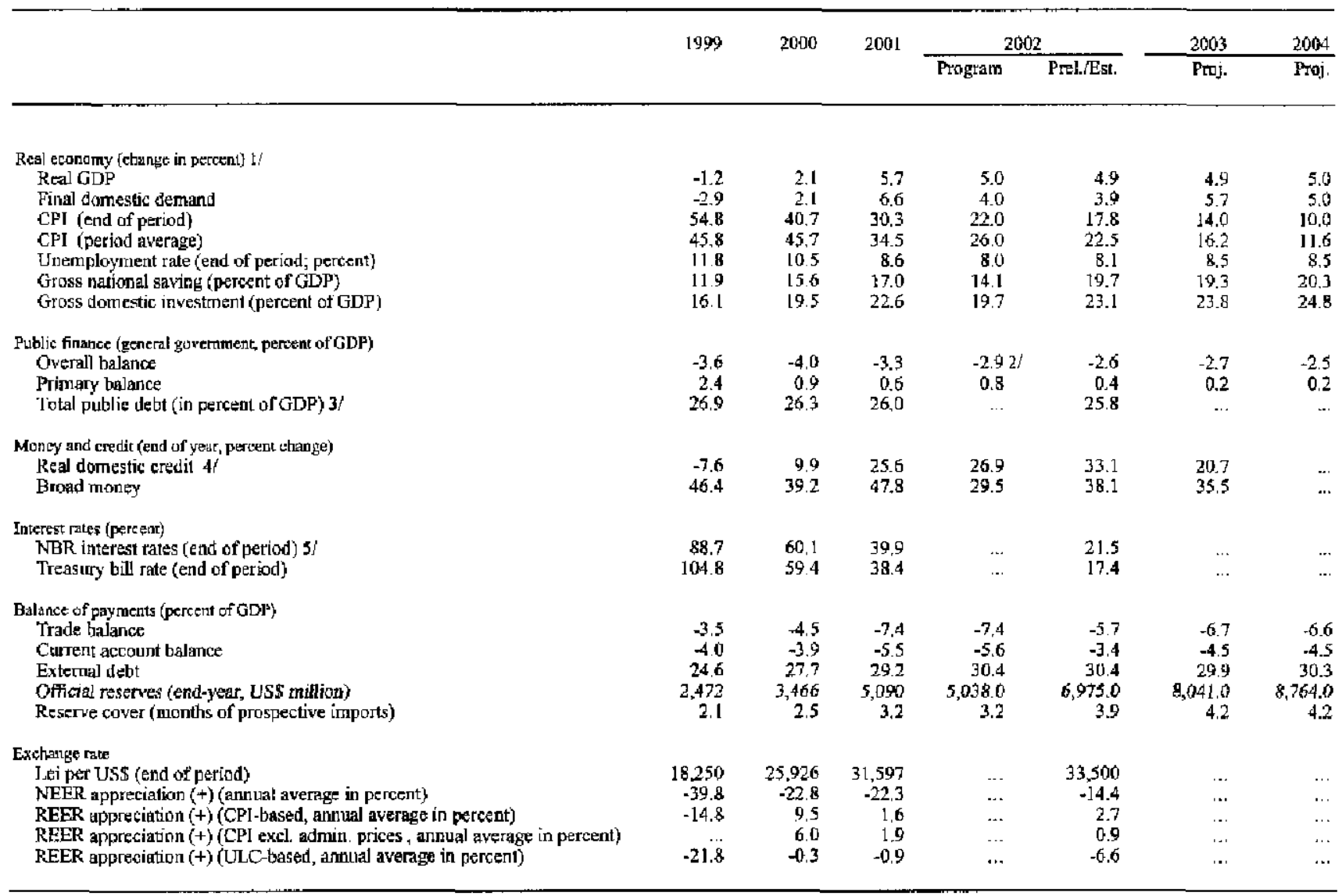

Sources; Romamian authorities; and Fund suffe estimatcs.

I/ In 2002, national accouluts data stanting in 1998 have been zcvisod dus to methodological change (adoption of ESA95 guidelines).

$2 /$ The original target for the nominal budget deliçit was eqgivalent to 3.0 penent of ODP. The upward revision of the nominal GDP was reflected in a lower deficit target in terms of GDP.

$3 /$ Including domcstic public debt and cxtema! public debt (public and puhlicly guaranteod).

4/ Credit to nongovenment soctor. From 1999 onwards, weighted average of neal lei credit growth and U.S. dollar-meacured forrign currency cte (bil growth.

5/ NBR deposit auction iocerest rate. 
Tuble 2. Rumantia: Balance of Payments, 2002-03 (In millıons of U.S. dollars)

\begin{tabular}{|c|c|c|c|c|c|c|c|c|c|c|c|}
\hline & \multicolumn{6}{|c|}{20025} & \multicolumn{5}{|c|}{2003} \\
\hline & $\begin{array}{l}\text { QT } \\
\text { A.GL }\end{array}$ & $\begin{array}{l}\text { Qf } \\
\text { Aet }\end{array}$ & $\begin{array}{l}\mathrm{Q}^{3} \\
\mathrm{~A}=\mathrm{H}\end{array}$ & $\begin{array}{l}\text { Dat } \\
\text { Aat. }\end{array}$ & Am. & Prog: & $\begin{array}{l}\mathrm{R}_{21} \\
\mathrm{P}_{\mathrm{roj}},\end{array}$ & $\begin{array}{l}\mathrm{Q}^{2} \\
\mathrm{P}_{\mathrm{TO}}\end{array}$ & $\begin{array}{l}\text { QS } \\
\text { Forj. }\end{array}$ & $\begin{array}{l}\text { Q9 } \\
\text { Pruj. }\end{array}$ & Proj. \\
\hline Currem aceoult & .277 & .627 & .21 & 647 & $-1,5 \pi$ & $-2,224$ & -422 & $.76 ?$ & -490 & -756 & $-2,3 \leqslant 2$ \\
\hline Recio on tontsul GDP & $-0,6$ & -1.4 & 0.0 & -1.4 & .3 .4 & 56 & -0.6 & -1.4 & -0.9 & -1.4 & -4.5 \\
\hline Tand balarse & 473 & -667 & -591 & $-B \mathbf{B}$ & $-2,692$ & $-3,039$ & -698 & -853 & -845 & $-1,126$ & $.3,525$ \\
\hline Eupurss & 2,900 & 3,319 & 3,764 & $3, \mathbf{8 8} 7$ & 11,870 & 17,699 & $x, 086$ & 3,640 & 4,174 & a:zas & $15,14,4$ \\
\hline Import6 & $\cdot 3,373$ & $-3,986$ & $-1,735$ & $-4,765$ & $-1 \mathrm{f}, \Delta \mathbf{2} 2$ & $-15,728$ & $-3,784$ & $-4,493$ & $-5,019$ & $.5,173$ & $-18,664$ \\
\hline Sarvices aneoum, thet & -102 & -255 & נ נו & -165 & -496 & -4.13 & 128 & 73.3 & 17 & 216 & .665 \\
\hline Peccipts & 497 & 648 & 792 & 762 & 2.699 & 2,587 & 5S4 & 746 & 878 & B日] & 2,981 \\
\hline Of whicht: Mtrentat & 39 & 44 & $\mathrm{~T} 7$ & 60 & 210 & 400 & il & 17 & 100 & T & 286 \\
\hline Paymeats & -600 & .903 & $-7 b 1$ & 4.911 & -165 & $-1,0200$ & -652 & $-1,0] 9$ & -866 & $-4,060$ & $-2,647$ \\
\hline of which buerest & -93 & -212 & -127 & .201 & .633 & .860 & .114 & -261 & $-15 \%$ & -247 & $-3+19$ \\
\hline Or'which. Inesest, net & -54 & .168 & -so & -141 & $41 ?$ & 460 & -64 & -104 & -56 & -169 & -493 \\
\hline Uarequñted transfirs ineti & 299 & 295 & 5.9 & 4003 & 1,536 & 1,178 & 404 & +2.3 & 423 & $\leq \$ 8$ & 1,838 \\
\hline$D / w a$ official & $: 4$ & 19 & 157 & $\pi 1$ & 28 & 190 & 31 & is & 101 & 117 & 264 \\
\hline viluate & 265 & $27 \dot{a}$ & 382 & 332 & 1,295 & 1,098 & $3 n$ & 408 & 322 & $\infty 71$ & $1, \$ i 4$ \\
\hline Capital azcous & $\$ 93$ & $\mathbf{s 2}$ & 647 & 1,686 & $3,70 ?$ & 3.580 & $7 N$ & 1,210 & 6,98 & $5 \pi$ & 3,188 \\
\hline Noert inver lment and ap pital transkers & 253 & 267 & 328 & 369 & 1,178 & 1,45? & 316 & 395 & 393 & 986 & 1,490 \\
\hline Parthofis inrestanes & $s$ & $*$ & -1 & $m$ & 124 & so & 25 & ie & 0 & $s$ & 50 \\
\hline Baprowing ty a cutlic secoor $\quad b$ & 167 & 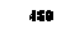 & 140 & 510 & 1,296 & 67. & 268 & 476 & as & 3.3 & $81 \%$ \\
\hline Loanus (net) & 10.3 & to & 80 & asu & 668 & 530 & 26E & 76 & $4 \mathbf{I}$ & 19 & 526 \\
\hline Disbureernent, & 399 & 341 & 409 & 847 & 1,928 & 1,670 & 567 & 353 & 350 & 499 & $1,76.9$ \\
\hline Of which: Direel debr & 114 & 160 & 193 & 3IS & 732 & B45 & 204 & 296 & 197 & 393 & 1,000 \\
\hline Of whtkh: Gavemmenn guaranieed & 225 & 232 & 2016 & $5: 42$ & $1,1 y_{4}$ & B30 & $36 y$ & 148 & 152 & 106 & 76,9 \\
\hline Ascortüzation & $-2+66$ & $-5 \phi s$ & -320 & -397 & $-1,259$ & $-1.1 \% 0$ & -300 & -278 & -308 & -308 & $.1,19.3$ \\
\hline Bunds (1net) & 64 & $4+1$ & 60 & 60 & 538 & 143 & D & Sint: & 0 & $-1 \leqslant 5$ & 242 \\
\hline UisbutemenfHF & 43 & 677 & 30 & 30 & 740 & 40 & 0 & 490 & 0 & o & 400 \\
\hline A:morization & 24 & .243 & 30 & 30 & $1+2$ & -297 & o & a & 0 & -1.58 & -158 \\
\hline Bontowing by a rivate sectn (nrt) & 11 & -35 & 99 & 5.99 & 694 & 399 & 121 & 379 & 144 & -14 & $6] 0$ \\
\hline Medium md locd term lasas (ner) & 11 & -35 & 99 & 549 & 624 & 399 & $|2|$ & $3 x y$ & la4 & -14 & (6) 0 \\
\hline Bikburcemerts & so & 176 & 315 & 762 & 1,313 & 1,075 & 299 & $5 \pi$ & 557 & 630 & 2,064 \\
\hline Androtisitisu & -70 & .811 & -216 & -213 & .710 & -676 & -178 & .190 & ] & -644 & $-[, 4] 4$ \\
\hline Trade cordit (hel) & 98 & 158 & 81 & 144 & $4 \$ 1$ & 0 & sin & 50 & 50 & .50 & 200 \\
\hline odver nturst-tersm [net] & 89 & 145 & $\eta$ & 147 & 438 & 0 & 50 & 50 & 30 & 50 & 200 \\
\hline \multicolumn{12}{|l|}{ Cap finzusieng wn ortizution } \\
\hline Nen etres and anissimas: & -269 & 258 & -194 & -733 & -917 & 300 & 0 & a & 0 & 0 & 0 \\
\hline OVefeql toplaner. & -13 & 47 & 432 & 206 & 1,198 & 387 & 3.48 & 446 & 328 & -18 & 826 \\
\hline Fingerras & 13 & $\rightarrow / s$ & -152 & $-y u b$ & $-1,198$ & $-3 y$ i & -448 & -446 & 248 & $16:$ & -65 \\
\hline Net forcign assete NBR (in crmase, -) & -214 & .756 & .508 & .499 & 1,977 & -507 & -299 & .526 & -308 & 118 & -950 \\
\hline Assets (Inerkst $\mathrm{P},-\rightarrow$ & -193 & $3 \mid 7$ & -604 & -331 & $-1,8.85$ & $-62 t$ & -231 & -968 & -427 & 160 & $-1,060 \%$ \\
\hline Liataile is & $2 \pi$ & $3 y$ & 96. & -128 & -97 & 114 & -8 & 42 & $\mathrm{L19}$ & d) & 110 \\
\hline $\mathbf{L M F}_{\text {, Let }}$ & -21 & -19 & 96 & .28 & E & 114 & -8 & 42 & 515 & -4 & 110 \\
\hline Purchests & 0 & o & ins & 0 & Ios & 211 & 0 & 71 & |A| & 0 & 212 \\
\hline Repurcbases & -81 & -99 & $-10^{\circ}$ & -28 & .98 & -97 & .8 & .29 & .22 & .43 & -102 \\
\hline Otherr, net & 0 & o & a & -100 & 1000 & 0 & 0 & J & 0 & 0 & o \\
\hline Shorthere & 0 & 0 & a & -100 & .100 & 0 & 0 & D & 0 & 0 & $a$ \\
\hline Mediton and lanf-1emm, ae: & 0 & 0 & 0 & 0 & a & 0 & 0 & a & 0 & 0 & 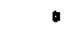 \\
\hline Distrinserments & 0 & B & 0 & 0 & 1) & $\mathbf{0}$ & 0 & a & * & $\mathbf{0}$ & . \\
\hline Repayments & 0 & - & 0 & 0 & 0 & 0 & 0 & a & (1) & 0 & - \\
\hline 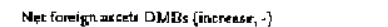 & 227 & $2 \mathbf{2 3}$ & 76 & $19 \mathrm{x}$ & 799 & -80 & -109 & 80 & sin & 69 & 120 \\
\hline Asset (ingrense, -1) & 167 & HLA & 6 & 146 & 433 & .80 & -139 & so & 50 & 39 & a \\
\hline Limbilkies & 60 & 169 & 70 & 47 & 346 & • & 30 & 30 & J* & 30 & 120 \\
\hline Shorterm & 69 & 7 & 60 & $\Rightarrow$ & 120 & I & 0 & 0 & - & 0 & a \\
\hline Medium- and long-werm, ner & -3 & g! & 20 & $12 \mathrm{~B}$ & 236 & I & 0 & 0 & - & 0 & a \\
\hline Port'ollo (net) & 0 & $\theta$ & a & 0 & - & 4 & 0 & 0 & - & $\square$ & a \\
\hline Find thing pap & 0 & 0 & 0 & 0 & 0 & 0 & 0 & 0 & in & 0 & a \\
\hline wo be sorexed by the Fund & & & & & & & & & & & \\
\hline Megrofandum itembi & & & & & & & & & & & \\
\hline Cureent acsour bala nee phe JDJ & $\ldots$ & $\ldots$ & $\ldots$ & $\ldots$ & 394 & -856 & $\ldots$ & $\ldots$ & $\ldots$ & $\ldots$ & .861 \\
\hline Retid to GDP & .. & $\ldots$ & $\ldots$ & $\ldots$ & -0.9 & -2.0 & $\ldots$ & $\ldots$ & $\ldots$ & ... & -1.6 \\
\hline Export groutb (percent) & 0.9 & 17,6 & 26.9 & 42.8 & $21: 3$ & 7.4 & 6.4 & 9.7 & 10,11 & 9.2 & 0.2 \\
\hline 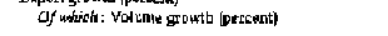 & -. & 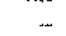 & $\ldots$ & - & 17.00 & 7.4 & $\ldots$ & $\ldots$ & . & 4 & 8.3 \\
\hline ahe unic พalue grow/to (percert) & $\ldots$ & -_- & $\ldots$ & w. & 9,13 & tol & $\ldots$ & $\ldots$ & ... & $\ldots$ & 0.9 \\
\hline Import growtb (percent) & 1.1 & 6.7 & 31.8 & 22.0 & 14.87 & 2.8 & 12.2 & 12.7 & 19.3 & 127 & 13.3 \\
\hline 1) which: Vol inde grownh pecoenti & $\ldots$ & $\ldots$ & $\ldots$ & $\ldots$ & 12,00 & 7.1 & $\ldots$ & $\ldots$ & $\ldots$ & $\ldots$ & 7.5 \\
\hline oiw unit value growth (perrent) & $\cdots$ & $m$ & $\ldots$ & $\ldots$ & 2.52 & D.T & $\ldots$ & $\ldots$ & $\ldots$ & $\ldots$ & 54 \\
\hline GDP growt & $\ldots$ & $\ldots$ & $\ldots$ & $\cdots$ & 7.7 & 7.7 & $\ldots$ & $\ldots$ & $\ldots$ & $\ldots$ & 7.3 \\
\hline ODP (in hillib: of of Iss) & $\cdots$ & $\ldots$ & $\ldots$ & $\ldots$ & 4.. 7 & 40,4 & $\cdots$ & $\ldots$ & $\ldots$ & $\ldots$ & 52.7 \\
\hline Terras of Lude (sercentuge thalnge) & $\ldots$ & $\ldots$ & $\ldots$ & $\ldots$ & 1.6 & -0.6 & $\ldots$ & $\ldots$ & $\ldots$ & $\ldots$ & 4.3 \\
\hline Externed decte (USS) & $\ldots$ & $\ldots$ & $\ldots$ & $\ldots$ & $10,7,787$ & 12,407 & $\cdots$ & $\ldots$ & $\cdots$ & $\ldots$ & 15,765 \\
\hline 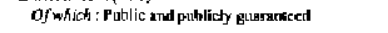 & $\cdots$ & $\ldots$ & $\ldots$ & $\ldots$ & 8,920 & 8,661 & $\ldots$ & $\cdots$ & $\cdots$ & $m$ & प्र,84; \\
\hline Entemal debu'OCPP (Eercert) & $\ldots$ &. & $\ldots$ & $\ldots$ & 90.4 & 30.4 & $\cdots$ & $\ldots$ & $\cdots$ & $\ldots$ & 29.9 \\
\hline Wet Exuenal Indebchess s ODP (percent) & $\ldots$ & $\ldots$ & $\ldots$ & $\ldots$ & 12.6 & 19.5 & $\ldots$ & $\ldots$ & $\ldots$ & $\ldots$ & 12.4 \\
\hline Debt sनrvige (UรS) & $\ldots$ & $\ldots$ & $\ldots$ & $\ldots$ & 2,961 & 3,070 & . & $\ldots$ & $\ldots$ & $\ldots$ & 3,666 \\
\hline Detbluerriute ralio & $\ldots$ & $\ldots$ & $\ldots$ & $\ldots$ & 18.3 & 20.1 & $\ldots$ & $\ldots$ & $\ldots$ & $\ldots$ & 20.8 \\
\hline Coves official reserves of the NGQ & 5,2563 & 6.0010 & 6,604 & 6,975 & 6,975 & 5,038 & 7,2016 & 2,774 & 8.202 & 8,041 & $8,0 \times 1$ \\
\hline (in monthe of importt of goods and secriaces) & $\ldots$ & .. & $\ldots$ & $\ldots$ & 3, & 3.2 & $\ldots$ & $\ldots$ & ... & $\ldots$ & 4.2 \\
\hline (w pereent of ebar-berwo debt') & $\cdots$ & $\ldots$ & $\cdots$ & $\cdots$ & 177.0 & 189.4 & $\cdots$ & $\cdots$ & $\ldots$ & $\cdots$ & 160.9 \\
\hline Net extemal firancing of the budger (USS mbi) & $\ldots$ & $\ldots$ & $\ldots$ & $\ldots$ & 684 & 751 & $\ldots$ & $\ldots$ & $\ldots$ & $\ldots$ & $\mathrm{T} 36$ \\
\hline Nombinal GDP \{USE,bh) & $\ldots$ & $\ldots$ & $\ldots$ & $\ldots$ & 45.7 & 40.8 & $\ldots$ & $\ldots$ & $\ldots$ & $\ldots$ & 527 \\
\hline
\end{tabular}

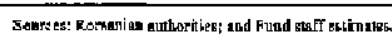


Table 3. Romania: Summary of Consolidated General Government, 1999-2003

\begin{tabular}{|c|c|c|c|c|c|c|c|c|c|c|c|c|c|c|c|c|}
\hline & \multirow{2}{*}{$\begin{array}{c}1999 \\
\text { Actual }\end{array}$} & \multirow{2}{*}{$\begin{array}{l}2000 \\
\text { Actual }\end{array}$} & \multirow{2}{*}{$\begin{array}{c}2001 \\
\text { Attual }\end{array}$} & \multicolumn{2}{|c|}{20062} & \multicolumn{3}{|c|}{2003} & \multirow{2}{*}{$\begin{array}{c}1999 \\
\text { Actual }\end{array}$} & \multirow{2}{*}{$\begin{array}{l}2000 \\
\text { Actual }\end{array}$} & \multirow{2}{*}{$\begin{array}{l}2001 \\
\text { Actual }\end{array}$} & \multicolumn{2}{|c|}{2002} & \multicolumn{3}{|c|}{2003} \\
\hline & & & & $\begin{array}{c}\text { Supplem. } \\
\text { budfer } \\
31-0 \text { tot-02 }\end{array}$ & $\begin{array}{c}\text { Actual } \\
\text { A/s/ } \\
21-\text { Feb-03 }\end{array}$ & $\begin{array}{c}\text { Budgel } \\
6 \text { t } \\
\text { 13-Now-0n } \\
\end{array}$ & $\begin{array}{l}\text { MOF/Slaff } \\
\text { estimate 6i } \\
\text { 21.Feb-03 } \\
\end{array}$ & $\begin{array}{l}\text { MOF/Staff } \\
\text { estimate } \bar{y} / \\
\text { 2I-Feb-03 } \\
\end{array}$ & & & & $\begin{array}{c}\text { Supplem. } \\
\text { budgel } \\
31-0 \mathrm{ct}-02 \\
\end{array}$ & $\begin{array}{c}\text { Acluaf } \\
4 / 5 t^{\prime} \\
21-\text { Feb-o3 } \\
\end{array}$ & $\begin{array}{c}\text { Budger } \\
6 \% \\
\text { 13-Nov-02 } \\
\end{array}$ & $\begin{array}{l}\text { MOE'Siff } \\
\text { estimate 6 } \\
\text { 2I-Feb-03 }\end{array}$ & $\begin{array}{l}\text { MOF/Senff } \\
\text { estimate 5/ } \\
21-\mathrm{F} \text { cb-0)3 }\end{array}$ \\
\hline & \multicolumn{8}{|c|}{ (in billions lei) } & \multicolumn{8}{|c|}{ (in percent of GDP) } \\
\hline Toral revenne and grants & 175,838 & 251.095 & 351,741 & 451,202 & 448.95 .4 & 562.457 & 561,339 & 567,968 & 31.9 & 31.2 & 30.1 & 29.8 & 29.7 & 310.6 & $\mathbf{3} 0.5$ & 30.9 \\
\hline Cursent & 173,337 & 249,445 & 351,108 & 450,499 & 447,987 & 561,202 & 539,884 & $\$ \$ 1,024$ & 31,8 & 31.1 & 30.1 & 29.8 & 29.6 & 30.5 & 30.4 & 30.5 \\
\hline Tax & 164.026 & 235,048 & 326,699 & 423,654 & 417,899 & 523,968 & 521,430 & $\$ 21,430$ & 30.1 & 29.2 & 28.6 & 28.0 & 27.6 & 28.5 & 28.4 & 28.4 \\
\hline Profits & 17,037 & 20,334 & 72,206 & 28,093 & 30,190 & 30,596 & 43,200 & 43,200 & 3.1 & 2.5 & 1.9 & 1.9 & 2.0 & 2.2 & 2.3 & 2.3 \\
\hline Wages and salaries $\mathrm{V}$ & 17,834 & 26,998 & 37,203 & 42,600 & 41,661 & 55,434 & 59,079 & 59.079 & 3.3 & 3.4 & 3.2 & 2.8 & 2.8 & 3.2 & 3.2 & 3.2 \\
\hline Social security $1 /$ & $57,66 ?$ & 86,557 & 125,106 & 169,628 & 361,600 & 195,760 & 185,760 & 185,760 & 10.6 & 10.8 & 10.7 & 11.2 & 100.7 & 10.5 & 10.1 & 0.1 \\
\hline Other direct trues & 8,276 & 8,081 & 13,025 & 33,020 & 14,637 & 19,151 & 19,346 & 19.346 & 1.5 & 1.0 & 1.1 & 0.9 & 1.0 & 1.0 & 1.1 & 1.1 \\
\hline YAT & 32,471 & 30,439 & $73+604$ & 103,366 & 104,495 & 124,572 & 133,000 & 133,000 & 6.0 & 6.3 & 6.3 & 6.8 & 6.8 & 6.8 & 7.2 & 7.2 \\
\hline Customs & 7,847 & 8,702 & $9,033.3$ & 9,000 & 9,362 & 8.163 & 8,163 & 8,163 & 1.4 & 1.1 & 0.8 & 0.6 & 0.6 & 0.4 & 0.4 & 0.4 \\
\hline Excisc & 16,958 & $20 ; 636$ & 27,293 & 33,792 & 32,4344 & 45,880 & 52,370 & 52,370 & 3.1 & 2.6 & 2.3 & 2.2 & 2.1 & 2.5 & 2.8 & 2.8 \\
\hline Other indireel twases & $5,93 ?$ & 13,301 & 19,224 & 24,155 & 23,521 & 31,412 & $20,5 \div 2$ & 20,512 & $1 . \mathrm{L}$ & 1.7 & 1.6 & 1.6 & 1.6 & 1.7 & 1.1 & 1.1 \\
\hline Nontax & $9,3]$ & 14,397 & 24,409 & 26,845 & $29,6 \times 8$ & 37,234 & 38,454 & 39,594 & 1.7 & 1.9 & $2 . \mathrm{l}$ & 1.8 & 2.0 & 2.0 & 2.1 & 2.2 \\
\hline Capital & 297 & 826 & 387 & 498 & 682 & 849 & 1,049 & 1,049 & a. 1 & a. 1 & 0.0 & 0.0 & 0.0 & 0.0 & 0.1 & 0.1 \\
\hline Grants & 204 & 325 & 246 & 205 & 685 & 405 & 405 & 5,895 & 0.0 & 0.0 & 0.0 & 0.0 & 0.0 & 0.0 & 0.0 & 0.3 \\
\hline Total expenditure & $193,56 ?$ & 283,351 & 389,321 & 494,402 & 488,780 & $6: 1,977$ & 610.859 & 617,560 & 35.5 & 35.3 & 33.4 & 32.7 & 32.3 & 33.3 & 33.2 & 33.6 \\
\hline Cunent & 177,835 & 255,275 & 351,794 & 445,310 & 439,036 & 541,467 & 539,804 & 546,506 & 32.6 & 31.8 & 30.1 & 29.4 & 29.0 & 29.4 & 29.4 & 29.7 \\
\hline Wages and salaries & 26,259 & 43,894 & 38,174 & 74,299 & 73,542 & 90,996 & 90,996 & 91,697 & 4.8 & 5.5 & 5.0 & 4.9 & 4.9 & 4.9 & 49 & 3.0 \\
\hline Materials and operating expenditures & 57,504 & 56,503 & 81,119 & $104,6 \mathrm{JZ}$ & 107,415 & {$[25,811$} & 125,755 & $131,4] 4$ & 10.5 & 7.0 & 6.9 & 6.9 & 7.1 & 6.8 & 6.8 & 7.1 \\
\hline ofw: health insurance & 22,597 & 24,597 & 36,779 & 47,924 & 47,678 & 55,184 & 55,184 & 55,184 & 4.1 & 3.1 & 3.2 & 3.2 & 3.2 & 3.0 & 3.0 & 3.0 \\
\hline laterest & 28.796 & 38,973 & 44.610 & 48,076 & 45,802 & 50,317 & 53,030 & 59,371 & 5.3 & 4.8 & 3.8 & 3.2 & 3.0 & 3.0 & 2.9 & 2.9 \\
\hline Subsidies and iransfers & 80,239 & 115,509 & 167,891 & 218,136 & 212,277 & 269,166 & 268,847 & 268,847 & 14.7 & 14.4 & 14.4 & 14.4 & 14.0 & 14.6 & 14.6 & 14.6 \\
\hline Subsidies & 9,303 & 17,581 & 24,294 & 30,795 & 29,865 & 39,616 & 34,066 & 34,056 & 1.7 & 2.2 & 2.1 & 2,0 & 2.0 & 1.8 & 1.9 & 1.9 \\
\hline Transfers & 70,936 & 98,324 & 143,597 & 187,341 & 182,412 & 235,550 & 234,781 & 234,781 & 13.0 & 12.2 & 12.3 & 12.4 & 12.1 & 12.8 & 12.8 & 12.8 \\
\hline a/w: Pensions & 37,854 & 53,579 & 79,578 & 103,926 & 102,154 & 124,964 & 120,964 & 120,964 & 6.9 & 6.7 & 6.8 & 6.9 & 6.8 & 6.8 & 6.6 & 6.6 \\
\hline Capital & 15,015 & 24,482 & 36,549 & 47,884 & $4 B, 579$ & 68,923 & 68,921 & 68,923 & 2.8 & 30 & 3.1 & 3.2 & 3.2 & 3.7 & 3.7 & 3.7 \\
\hline Lending minus repaygrmeals & 717 & 3,594 & 978 & 1,208 & 1,165 & 1,586 & 2,131 & 2,131 & 0.1 & 0.4 & 0.1 & 0.1 & 0.1 & 0.1 & 0.1 & 0.1 \\
\hline Overall babnce (cash, inchuding grants) & $-19,729$ & $-32,256$ & $-37,580$ & $-43,200$ & $.39,827$ & $.49,520$ & $-49,520$ & $-49,599$ & -3.6 & -4.0 & -3.2 & -2.9 & -2.6 & -2.7 & -2.7 & -2.7 \\
\hline Financing & 18,080 & 28,322 & 36,948 & 43,200 & 34,818 & 49,520 & 49,520 & 49,592 & 3.3 & 3.5 & 3.2 & 2.9 & 2.3 & 2.7 & 2.7 & 2.7 \\
\hline Domestif & 8,614 & $-1,913$ & 10,730 & 13,6013 & 4,383 & 18,590 & 18,311 & 18,383 & 1.6 & -0.2 & 0.9 & 0.9 & 0.3 & 2.0 & 1.0 & 1.0 \\
\hline Extemal & 2,360 & 22,575 & 20,104 & 23,497 & 22,826 & 25,030 & $25,28 y$ & 25,209 & 0.4 & 2.8 & 1.7 & 1.6 & 1.5 & 1.4 & 1.4 & 1.4 \\
\hline Privatization proceeds & 6,859 & 5,062 & 3,460 & 4,000 & 5,532 & 4,000 & 4,000 & 4,000 & 1.3 & 0.6 & 0.3 & 0.3 & 0.4 & 0.2 & 0.2 & 02 \\
\hline Barth asset reosyeries & 247 & 2,597 & 2,654 & 2,100 & 2,077 & 1,900 & 2,000 & 2,000 & 0.0 & 0.3 & 0.2 & 0.1 & 0.1 & 0.1 & 0.1 & 0.1 \\
\hline Diserepaney $2 s$ & $-1,649$ & $-3,934$ & -632 & 0 & $-5,009$ & 0 & $a$ & 0 & -0.3 & -0.5 & -0.1 & 0.0 & -0.3 & 0.0 & 0.0 & 0.0 \\
\hline Memorandum itenes: & & & & & & & & & & & & & & & & \\
\hline Primary ckpenditurc $3 /$ & 160,956 & 244,167 & 344,711 & $\begin{array}{l}446,326 \\
307,734\end{array}$ & 442,979 & 557,660 & 557.829 & 564,189 & 29.5 & 30,4 & 29.5 & 29.5 & 29.3 & $\begin{array}{l}30.3 \\
20.5\end{array}$ & 30.3 & $\begin{array}{l}30.7 \\
26.8\end{array}$ \\
\hline Prinary clurent expeaditure 3 & 149,039 & 216,302 & 307,184 & 397,234 & $\begin{array}{r}393,234 \\
5,975\end{array}$ & 487,150 & 486,775 & 493,133 & 27.3 & 26.9 & $\begin{array}{r}26.3 \\
0.5\end{array}$ & $\begin{array}{r}26.3 \\
0.3\end{array}$ & $\begin{array}{r}26.0 \\
0.4\end{array}$ & $\begin{array}{r}26.5 \\
0.3\end{array}$ & $\begin{array}{r}26.3 \\
0.2\end{array}$ & 0.2 \\
\hline Primary balance (cash, including granty) & $\begin{array}{r}12,883 \\
545730\end{array}$ & $\begin{array}{r}6,928 \\
803773\end{array}$ & $\begin{array}{r}7,030 \\
167,247\end{array}$ & $\begin{array}{r}4,876 \\
1.512,257\end{array}$ & $\begin{array}{r}5,975 \\
1,512,257\end{array}$ & $\begin{array}{r}4,797 \\
838,707\end{array}$ & $\begin{array}{r}3,510 \\
1,878,707\end{array}$ & $\begin{array}{r}3,779 \\
1.838,707\end{array}$ & $\begin{array}{r}2.4 \\
345.730\end{array}$ & $\begin{array}{r}0.9 \\
803,773\end{array}$ & $\begin{array}{r}0,6 \\
1,167,243\end{array}$ & $\begin{array}{r}0.3 \\
1512,257\end{array}$ & $\begin{array}{r}04 \\
1,512,257\end{array}$ & $3,838,707$ & $1,838,707$ & $1,838,7077$ \\
\hline
\end{tabular}

Sources: Minisiry of Public Finarlew; and Furd ste ff estimates.

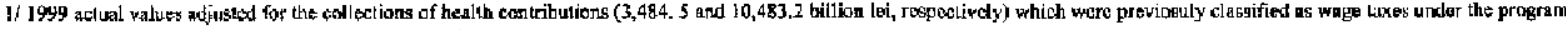
2it Oretall balance (cash, including grants) pis financing.

3/ Excluding EU grants and cash expenditure for bank restructuring.

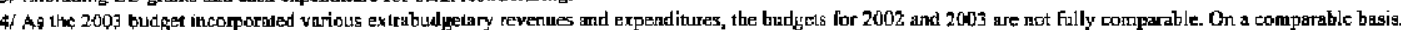

revenues and expenditure in the 2002 budget are higher by about 0.3 percent of GDF, leaving the overal belence unchenged.

5) Including National Administration of Rosds.

6/ Exclucting Natitrmal Adruinistration of Roads. 
Table 4. Romania: Monetary Survey, 2001-03

(In billions of lei, actual/program exthange rates)

\begin{tabular}{|c|c|c|c|c|c|c|c|c|c|c|c|c|c|}
\hline & \multicolumn{2}{|l|}{2001} & \multicolumn{7}{|c|}{2002} & \multicolumn{4}{|c|}{2003} \\
\hline & $\begin{array}{c}\text { Decenber } \\
\text { ectual }\end{array}$ & $\begin{array}{l}\text { March } \\
\text { 피aan }\end{array}$ & $\begin{array}{l}\text { March } \\
\text { actual }\end{array}$ & $\begin{array}{c}\text { Juno } \\
\text { tEr. Diogram }\end{array}$ & $\begin{array}{l}\text { June } \\
\text { Thetual } \\
\end{array}$ & $\begin{array}{c}\text { Sepiennber } \\
\text { Iev. program }\end{array}$ & $\begin{array}{l}\text { Septermber } \\
\text { actual }\end{array}$ & $\begin{array}{c}\text { December } \\
\text { rev_ program }\end{array}$ & $\begin{array}{c}\text { Docember } \\
\text { actual }\end{array}$ & $\begin{array}{l}\text { March } \\
\text { pougram }\end{array}$ & $\begin{array}{c}\text { June } \\
\text { purngram }\end{array}$ & $\begin{array}{c}\text { September } \\
\text { mengram }\end{array}$ & $\begin{array}{c}\text { December } \\
\text { program }\end{array}$ \\
\hline \multicolumn{14}{|l|}{ Monelary Survey } \\
\hline Net foreign assets (including valuation changes) & 171,866 & 169,198 & 178,368 & 201,736 & 197,564 & 202,910 & 209,139 & $210_{z}^{913}$ & 222,271 & 235,077 & 263,662 & $281,74]$ & 284,507 \\
\hline In millians of L.S. dollars & 5,439 & 5,074 & 5,424 & 5,885 & 5,901 & 5,910 & 6,327 & 5,955 & 6,635 & 7,038 & 7,524 & 7,804 & 7,685 \\
\hline Of which: Comunercial banks & 866 & $\mathrm{BOA}$ & 639 & 325 & 356 & 236 & 280 & 145 & 87 & 196 & 116 & 36 & -33 \\
\hline NBR & 4,573 & 4,266 & 4,785 & $s, 560$ & 5,545 & 5,674 & 6,047 & 5,810 & 6,548 & 6,842 & 7,408 & 7,768 & 7,718 \\
\hline Net donestic assets & 98,646 & 89,723 & 96,958 & 95,328 & 109,347 & 108,238 & 108,193 & 139,350 & 151,440 & 150,629 & 151,848 & 166,250 & 221,690 \\
\hline Domestic credit & 143,245 & 149,943 & 155,234 & 185,881 & 164,421 & 177,450 & 170,243 & 204,530 & 200,358 & $216, x 14$ & 222,297 & 237,766 & 268,180 \\
\hline Gowernmenl & 24,990 & 30,453 & 24,997 & 33,659 & 17,154 & 17,220 & 13,241 & 26,667 & 20,732 & 25,004 & 14,622 & 17,358 & 28,150 \\
\hline Of which: T-bils far bank restructuring $1 /$ & $16,31.0$ & 14,294 & 16,632 & 17,449 & $L 6,392$ & 13,523 & 22,153 & 8,621 & 7,557 & 6,977 & 2,762 & 1,615 & 685 \\
\hline Non-govenment & 118,254 & 119,490 & 130,237 & 152,223 & 147,267 & 160,230 & 157,003 & 177,863 & 179,626 & $191, \$ 10$ & 207,676 & 220,408 & 240,030 \\
\hline In forejgn currency & 70,721 & 69,593 & 78,627 & 96,846 & 94,486 & 101,843 & 98,923 & 111,478 & 112,898 & 117,717 & 132,290 & 139,177 & 148,273 \\
\hline (in millions of U.S. dollats) & 2,238 & 2,087 & $2,39 !$ & 2,825 & 2,822 & 2,966 & 2,293 & 3,147 & $\mathbf{3 , 3 7 0}$ & 3,525 & 3,715 & 3,855 & 4,005 \\
\hline In lei & 47,533 & 49,897 & 51,610 & 55,377 & 52,781 & $58,38 \mathrm{R}$ & 58,079 & 66,385 & 66,729 & 73,593 & 75,386 & $81,23 \mathrm{~L}$ & $91,75 ?$ \\
\hline Other iterns net & $-44,598$ & $-60,270$ & $-58,276$ & $-90,553$ & $-61,074$ & $-69,212$ & $-62,050$ & $-65,180$ & 48,918 & $.65,685$ & $.70,449$ & $-71,516$ & $-46,430$ \\
\hline Broad tnoney & 270,512 & 258,921 & 275,326 & 297,063 & 300,912 & $311,14 k^{\circ}$ & 317,332 & 350,263 & 373,711 & 385,706 & 415,510 & $447,99 \mathrm{~L}$ & 506,196 \\
\hline Of which : Lei denominated (M2) & 154,728 & 142,875 & 157,746 & 171,241 & 176,348 & 183,755 & 189,458 & 217,429 & 226,900 & 235,396 & 256,049 & 280,100 & 326,627 \\
\hline Cunsency in circulation & 35,635 & 34,602 & 33,416 & 36,956 & 39,615 & 41,741 & 42,334 & 46,077 & 45,577 & 45,530 & $51,5 n+1)$ & 56,060 & 60,042 \\
\hline Lei depnoits & 119,092 & $11 \pm, 273$ & 124,330 & 134,285 & 136,733 & 142,014 & 147,124 & 171,352 & 181,322 & 189,866 & 204,549 & 224,040 & 266,586 \\
\hline For eign currency deposits & 115,784 & 116,046 & 117,580 & 125,823 & 124,564 & 127,393 & 127,874 & $I 32,834$ & 146,812 & 150,310 & 159,461 & 167,892 & 179,569 \\
\hline In millions of U.S. dollaws & 3,6664 & 3,480 & 3,575 & 3,670 & 3,721 & 3,710 & 3,869 & 3,750 & 4,382 & 4,500 & 4,550 & 4,650 & 4,850 \\
\hline \multicolumn{14}{|l|}{ Memorandum items: $\mathbb{3} /$} \\
\hline Brod momey $(\mathrm{M} 2 \mathrm{X})$ growth & 45.2 & 35.2 & 43.7 & 42.5 & 44.3 & 32.3 & 35,0 & 29.5 & 38.1 & 40.1 & 38.1 & 41.2 & 35.5 \\
\hline NFA coniribution & 55.9 & 31.9 & 36.7 & $3 i .5$ & 29.5 & 20.0 & 22.6 & 14.4 & 18.6 & 20.6 & 22.0 & 22.9 & 16.7 \\
\hline NDA contribution & -9.8 & 3.2 & 7.0 & 11.0 & $\mathrm{I} 4.8$ & 12.3 & 12.3 & 15.9 & 19.5 & 10.5 & 16.1 & 18.3 & 18.8 \\
\hline Lei-denominated mnney growli (M2) & 40.4 & 31.9 & 45.7 & 44.4 & 48.7 & 43.2 & 47.6 & 40.5 & 46.6 & 49.2 & 45.2 & 47.8 & 44.0 \\
\hline Growth of eurrency in circulation & 38.4 & 32.9 & 40.6 & 24.7 & 33.6 & 27.9 & 29.7 & 29.3 & 27.9 & 36.3 & 30.0 & 32.4 & 31.7 \\
\hline Real broad money grwwh & 12.2 & 5.6 & 14.9 & 14.8 & 6.4 & 7.6 & 12.7 & 6.1 & 17.2 & 19.6 & 19.0 & 21.1 & 18.8 \\
\hline Real lei-denominated money growth (M2) & 7.8 & 3.1 & 16.4 & 16.4 & 19.9 & 16.4 & 23.3 & 15.2 & 24.4 & 27.4 & 25.2 & 26.8 & 26.3 \\
\hline Real growth of culritency in sirculation & 6,3 & 3.8 & 12.3 & 0.5 & 7.7 & 4.0 & 8.3 & 6.0 & 8.5 & 16.3 & 12.1 & 13.6 & L.5.6 \\
\hline Growth of les credit to nongorernment, adjusted $2 t$ & 56.3 & 49.4 & 54.5 & 48.7 & 41.7 & 39.4 & 38.6 & 39.7 & $40, A$ & 42.6 & 42.8 & 39.9 & 37.5 \\
\hline Growth of lei credil to nongurenument, adjusted, real $2 /$ & 20.0 & 16.7 & 23.5 & 19.8 & $\$ 4.3$ & 13.3 & 15.7 & 14,5 & 19.1 & 21.8 & 23.1 & 20.0 & 20.6 \\
\hline Grouth of foreign currency credit, adjusted, in IISS $2 /$ & 33.2 & 12.7 & 31.0 & 40.1 & 40.4 & 42.7 & $45: 2$ & 34.3 & 41.5 & 39.9 & 33.2 & 27.7 & 20.8 \\
\hline Growth of eredit to nongovemnent, composite $2 / 4$ & 28.0 & 14.5 & 28.1 & 32,6 & 31.1 & 32.0 & 34.4 & 26.9 & 33.1 & 32.8 & 29.6 & 24.9 & 20.7 \\
\hline CPI inflation & 30.3 & 28.0 & 25.1 & 24.1 & 24.0 & 23.0 & 19.8 & 22.0 & 17.8 & 17.1 & 16.0 & 16,6 & 14.0 \\
\hline M2X (Broad Money) veloeity & 4.8 & 5.3 & 5.2 & 5.0 & 5.0 & 5.0 & 4.8 & 4.7 & 4.3 & 4.5 & 4.4 & 4.2 & 3.8 \\
\hline M2 (Domestic Broad Maney) velocity & 8.4 & 9.6 & 9.0 & 8.6 & 8.5 & 8.4 & 8.1 & 7.6 & 7,1 & 7.4 & 7.1 & 6.6 & 5.9 \\
\hline M2X+Tbill veloeity & 43 & 4.7 & 4.5 & 4.4 & 4.4 & 4.3 & 4.2 & 4.1 & 3.9 & 4.1 & 4.0 & 3.8 & 3.5 \\
\hline M2X+Tbill growth & 49.3 & 34.0 & 43.9 & 41.4 & $\mathbf{4 3 . 5}$ & 33.9 & 35.3 & 31.7 & 37.3 & 36.4 & 33.7 & 36.4 & 32.6 \\
\hline
\end{tabular}

Sources: Romanian authorities; and Fund staff estimates.

$1 /$ Bonds issued to restructure Bancorex and Batwa Agricola.

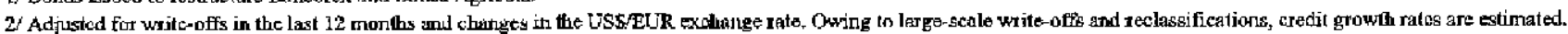

3/ All thanges are 12-month rates of change, unless otherwise indicated.

$4 /$ Real lei credit growth and foreign charrency credil growth, weighted by their respective shares.

CInternational Monetary Fund. Not for Redistribution 
Table 4. (continued) Romania: Balance Sheet of the National Bank, 2001-03 if (In billions of lej, actual/program exchange rates, monthly averages)

\begin{tabular}{|c|c|c|c|c|c|c|c|c|c|c|c|c|c|}
\hline & \multicolumn{2}{|l|}{2001} & \multicolumn{7}{|c|}{2002} & \multicolumn{4}{|c|}{2000} \\
\hline & $\begin{array}{c}\text { December } \\
\text { actual }\end{array}$ & $\begin{array}{l}\text { March } \\
\text { program }\end{array}$ & $\begin{array}{l}\text { March } \\
\text { Batual }\end{array}$ & $\begin{array}{c}\text { June } \\
\text { rev. program } \\
\end{array}$ & $\begin{array}{c}\text { Juns } \\
\text { actual } \\
\end{array}$ & $\begin{array}{l}\text { Saptember } \\
\text { rev. proggan }\end{array}$ & $\begin{array}{l}\text { September } \\
\text { actual }\end{array}$ & $\begin{array}{l}\text { December } \\
\text { rev. progran }\end{array}$ & $\begin{array}{c}\text { December } \\
\text { aotual }\end{array}$ & $\begin{array}{c}\text { March } \\
\text { prograrn }\end{array}$ & $\begin{array}{c}\text { June } \\
\text { grogramn }\end{array}$ & $\begin{array}{l}\text { Septensber } \\
\text { progiam }\end{array}$ & $\begin{array}{c}\text { December } \\
\text { programa }\end{array}$ \\
\hline \multicolumn{14}{|l|}{ National Bank of Romania } \\
\hline $\begin{array}{l}\text { Net foreign assels (including valuation) 2f } \\
\text { In millions of U.S. dollers }\end{array}$ & $\begin{array}{r}114,890 \\
3,641\end{array}$ & $\begin{array}{r}118,385 \\
3,575\end{array}$ & $\begin{array}{r}127,410 \\
3,889\end{array}$ & $\begin{array}{r}160,612 \\
4,716\end{array}$ & $\begin{array}{r}157,476 \\
4,716\end{array}$ & $\begin{array}{r}160,728 \\
4,687\end{array}$ & $\begin{array}{r}166,757 \\
5,017\end{array}$ & $\begin{array}{r}169,768 \\
4,823\end{array}$ & $\begin{array}{r}175,723 \\
5,221\end{array}$ & $\begin{array}{r}181,896 \\
5,486\end{array}$ & $\begin{array}{r}210,180 \\
6,039\end{array}$ & $\begin{array}{r}228,988 \\
5,374\end{array}$ & $\begin{array}{r}231,536 \\
6,274\end{array}$ \\
\hline Net domestic assets & $-48,929$ & $-56,184$ & $-59,972$ & $-85,673$ & $-82,467$ & $-79,768$ & $-84,518$ & $-80,571$ & $-83,337$ & $-85,740$ & $-106,780$ & $-113,972$ & $-106,679$ \\
\hline Total credit & $-21,036$ & $-22,097$ & $-28,853$ & $.55,216$ & $.52,651$ & $-56,077$ & $-56,429$ & $-56,711$ & $-55,273$ & $-49,454$ & $-72,426$ & $-81,450$ & $-75,126$ \\
\hline NBR refitinncitg (inchuding 1o DGF and Lat 101\} & 4,900 & 4,693 & 4,684 & 4,655 & 4,626 & 3,794 & 3,751 & 3,161 & 3,114 & 3,114 & 2,781 & 2,781 & $2,14 \%$ \\
\hline NER refinamcing to litigous dcbtor & 284 & 284 & 284 & 284 & 284 & 284 & 284 & 284 & 284 & 284 & 284 & 284 & 284 \\
\hline NBRe credit line in Deposit Giatantee Fund & 3,510 & 3,547 & 3,503 & 3,510 & 3,481 & 3,510 & 3,467 & 2,877 & 2,830 & 2,830 & 2,497 & 2,497 & 1,864 \\
\hline Law 101 (Bancorex) \& Special credit to BA & $\$, 105$ & 861 & 898 & 861 & 861 & 0 & 0 & 0 & 0 & 0 & a & 0 & 0 \\
\hline Deposit taking operations & $.24,835$ & $-25,903$ & $-33,956$ & 39,689 & $.38,744$ & $-45,543$ & $-49,659$ & $-50,257$ & $-50,910$ & $-47,914$ & $.57,343$ & $-69,192$ & $-64,884$ \\
\hline Reverse repo & $-1,058$ & $-4,313$ & $-1,974$ & -819 & .790 & -819 & -928 & -819 & -48 & 0 & 0 & 0 & $\mathbf{0}$ \\
\hline Cresit to covernthetr. & .43 & 3,427 & 2,393 & $-19,363$ & $-17,743$ & $-13,509$ & $.0,593$ & $-8,816$ & $-7,429$ & $-4,654$ & $-17,864$ & $-16,039$ & $-12,390$ \\
\hline 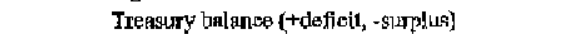 & $-7,762$ & $-4,776$ & $-4,746$ & $-25,577$ & $-24,776$ & $-18,071$ & $-14,973$ & $-11,436$ & $-9,916$ & $-6,916$ & $-17,864$ & $-16,039$ & $-12,390$ \\
\hline Treasury overdedf & $-3,158$ & $-1,299$ & $-3,511$ & $-3,168$ & $-3,168$ & $-3,168$ & $-9,054$ & $-3,168$ & $-3,693$ & $-3,693$ & $-3,693$ & $-3,693$ & $-3,693$ \\
\hline Treasury deponsits & 4,605 & 3,477 & 1,235 & 22,410 & $21,60 \mathrm{~B}$ & 14,903 & 5,919 & 8,268 & 6,223 & 3,223 & 14,170 & 12,346 & 8,696 \\
\hline Other ilems net & $-27,893$ & $-34,087$ & $-31,119$ & $-30,457$ & $-29,816$ & $-23,692$ & $-28,089$ & $-23,861$ & $-28,064$ & $-36,285$ & $-34,354$ & $-32,522$ & $-31,549$ \\
\hline Lei Irsejve money $3 / 6 t$ & 65,961 & 62,202 & 67,438 & 74,938 & 75,009 & 80,360 & 81,639 & 89,197 & 92,385 & 96,157 & 103,401 & 115,016 & 124,861 \\
\hline Curnency & 37,747 & 32,714 & 35,407 & 39,499 & 39,495 & 43,721 & 44,727 & 48,072 & 50,592 & 48,571 & 52,114 & 58,807 & 63,416 \\
\hline Barik deposits $6 r$ & 28,213 & 29,487 & 32,031 & 35,439 & 35,514 & 36,639 & 36,912 & 41,125 & 41,793 & 47,586 & 51,287 & $56 ; 209$ & 61,445 \\
\hline \multicolumn{14}{|l|}{ Menorandum items: $4 /$} \\
\hline Lai reserve money growth & 28.6 & 31.2 & 42.3 & 41.2 & 41.3 & 37.3 & 39.5 & 41.4 & 40.1 & 42.6 & 37.9 & 43. 1 & 35.2 \\
\hline NFA contribution & 131.9 & 129.5 & 148.6 & 153.5 & 147.6 & 103.0 & 113.3 & 92.8 & 922 & 80.8 & 70.3 & 85.7 & 60.4 \\
\hline NDA contribution & -1032 & $-9 \mathrm{~B} .3$ & -106.3 & -112.3 & -106.3 & -65.7 & .73 .8 & -51.5 & -52.2 & -38.2 & -32.4 & -42.6 & -253 \\
\hline Real reserve muney growth (adjusted) if & 6.9 & 2.9 & 14.2 & 24.3 & 24.6 & 160 & 20.9 & 16.2 & 19.2 & 21.8 & 18.8 & 22.8 & 18.6 \\
\hline Adjusted lei reserve money growth si 6 ; & 393 & 32.7 & 42.9 & 54.3 & 54.5 & 42.7 & 44.8 & 41.7 & 40.5 & 42.5 & 37.9 & 43.1 & 35.2 \\
\hline NDA contribution & .89 .7 & -87.1 & -93.3 & -99.5 & -529 & -55.4 & -63.1 & -43.5 & -44.3 & -43.6 & -38.3 & -47.4 & -31.5 \\
\hline NFA contribuxion & 128.9 & 111.7 & 136.2 & 153.3 & 147.4 & 98.1 & 1079 & 85.2 & 84.9 & 73.7 & 64.2 & 78.5 & 55.4 \\
\hline CPI inflation & 30.3 & 28.0 & 25.1 & 24.1 & 24.0 & 23.0 & 19.8 & 22.0 & 17.8 & 17.1 & 16.0 & 16.6 & 14.0 \\
\hline
\end{tabular}

Surmtes: Romanian authorities; and Fund staff estimates.

1/ For progran punpogets, all vahues are defined on a monthly sverage basis.

$2 /$ Progran definition exchudes deposits of conmerial benks to meel required reserves against foreigh currency deposits. This, and the fact thet the figures hexe are munthly averages, explain the differences from the monetary suruey. 3/ Indicative targds based on which the NDA PC is set.

4/ All changes are 12-munth tales of change, unlegs otherwise indicateri.

5/ Adjusted for both (i) shortialls in reported reserve money, and (ii) changes in minimumn teserve requirements.

(i) Shortfalls in reported reserve money occured in 1999 and early 2000 owing to failure of sume banks to observe the reserve requirements and the time lag givon to BCR to absorb certuin

transtered depongits frong the fermer Bancorex in the first part of 2000. Small deficiencies persisted throughout 2000 and 2001 and disappenred singes mid-2002

(ii) Minimum reserve requirement far lei depasits was decreastal in 2001 from 30 pacent in 27 percent on July 1 and further to 25 percent on October 1 .

In 2002, further cils were made (to 22 percent in Afril and to 18 percenl in December).

6/ Adjusted to corregpond to in 25 percent treserverequirenient. 
Table 5. Implicit Subsidies and Losses in the Energy Sector 2000-04

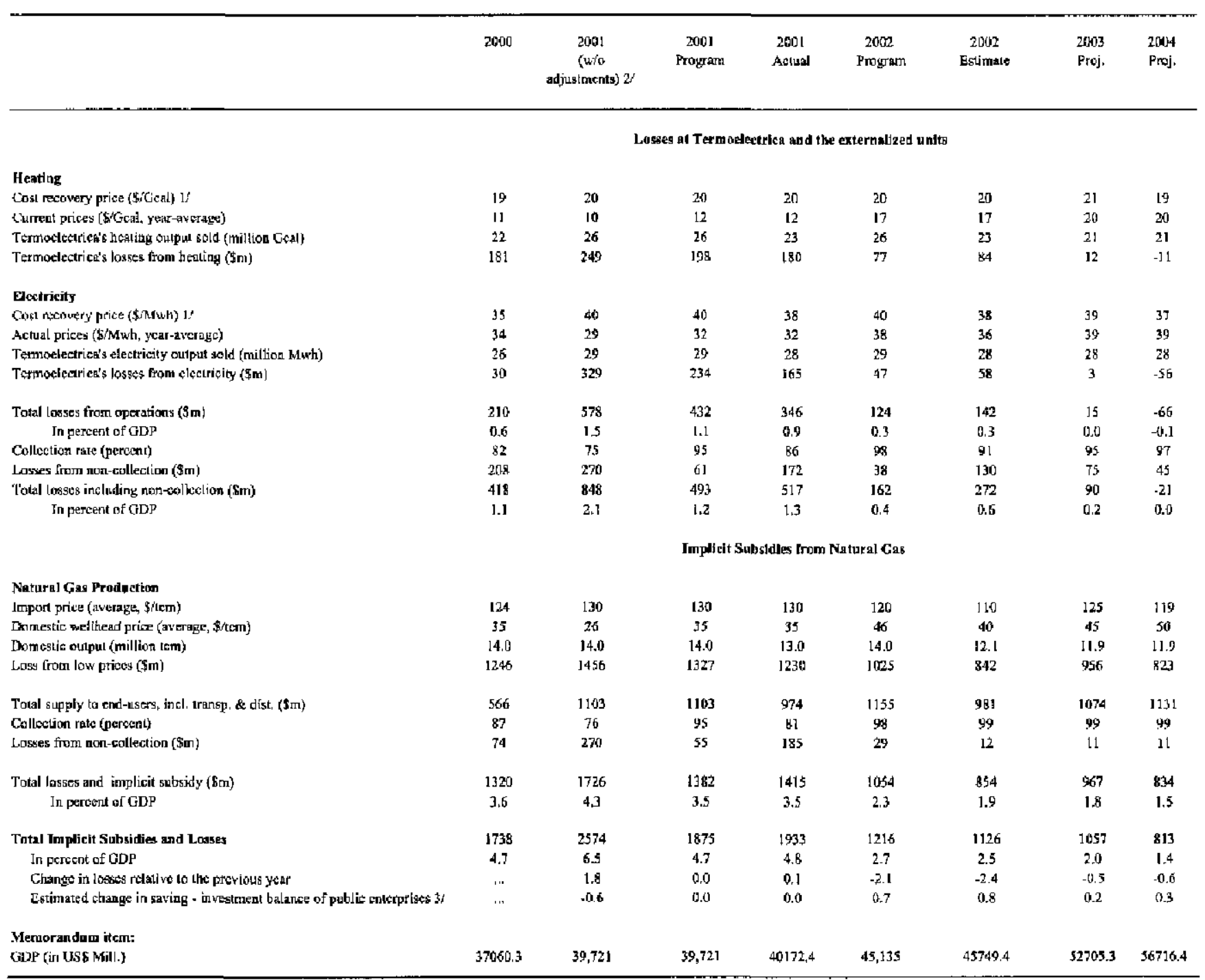

Sources: ANRE (electricily regubtory agency), Termoelestrice and Distrigaz Nord and S Sal; Workd B ank; Fund staff estimates.

1/. Cost recovery price estimates are based on WEO fut price jiojections, as of March 14, 2003.

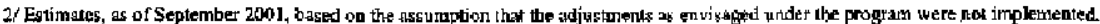

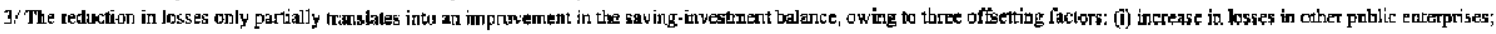

(ii) higher tax payments of the energy seteler, (iji) higher investenenti in the energy seckr. For 2003 and 2004 , this elfect is estimated at $1 / 2$. 
Table 6. Romania: Mediun-Term Balance of Payments Outlook, 2000-06

(In millions of L.S. dallars)

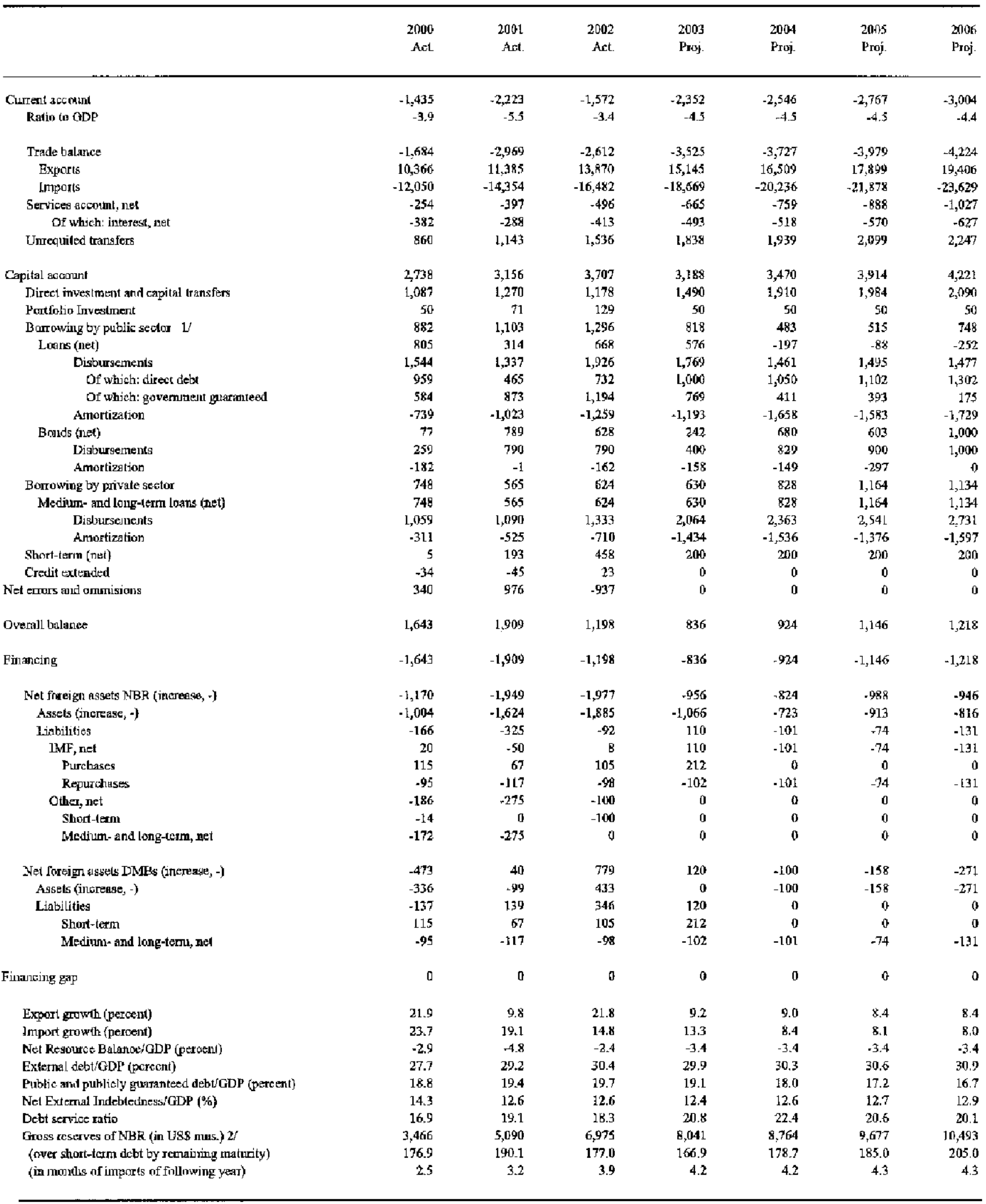

Sources: Romanian authortues; and Fund staff estimates.

$1 /$ Includijug public enterprises.

2 Ineludes gold. 
Table 7. Romania: External Fìnancing Requirements and Sources, 1999.2003

(In millions of U.S. dollars)

\begin{tabular}{|c|c|c|c|c|c|}
\hline & 1999 & 2000 & 2001 & 2002 & $\begin{array}{l}2003 \\
\text { Proj. }\end{array}$ \\
\hline Gross financing requirements & 3,742 & 4,388 & 5,987 & 5,352 & 6,305 \\
\hline External current account deficit & 1,446 & 1,435 & 2,223 & 1,572 & 2,352 \\
\hline Debt amortization & 2,244 & 1,518 & 1,924 & 2,230 & 2,785 \\
\hline Public sector & 1,610 & 1,207 & 1,399 & $1,52 \mathrm{~J}$ & 1,351 \\
\hline of which: Bonds and notes & 747 & 354 & 276 & 162 & 158 \\
\hline Private sector & 634 & 311 & 525 & 710 & 1,434 \\
\hline Repayment of arrears & 0 & 0 & 0 & 0 & 0 \\
\hline Gross reserves accumulation & -87 & 1,340 & 1,723 & 1,452 & 1,066 \\
\hline IMF repurchases and repayments & 139 & 95 & 117 & 98 & 102 \\
\hline Avallable financing & 3,203 & 3,828 & 5,819 & 5,078 & 5,818 \\
\hline FDl and capital transfers (net) & 1,070 & 1,087 & 1,270 & 1,178 & 1,490 \\
\hline Public scetor borrowing & 815 & 1,458 & 2,127 & 2,548 & 1,893 \\
\hline Loans & 815 & 1,199 & 1,337 & 1,758 & 1,493 \\
\hline Bonds & 0 & 259 & 790 & 790 & 400 \\
\hline Private sector borrowing & 780 & 922 & 1,229 & 1,679 & 2,184 \\
\hline Other flows $1 /$ & 538 & 361 & 1,194 & -327 & 250 \\
\hline Financing gap & 539 & 560 & 168 & 273 & 488 \\
\hline Balance of payments financing & 539 & 560 & 167 & 273 & 488 \\
\hline IMF & 73 & 115 & 67 & 105 & 212 \\
\hline Other 2/ & 466 & 445 & 100 & 168 & 276 \\
\hline Accumulation of arrears (exceptional) & 0 & 0 & 0 & 0 & 0 \\
\hline Residual financing gap & 0 & 0 & $\mathbf{I}$ & 0 & 0 \\
\hline
\end{tabular}

Sources: Romanian authorities; and Fund staflestimates.

1/ Includes all other net tinancial flows, and errors and omissions.

2/ Includes World Bank PSALZ and EU Joans. 
Table 8. Romania: Indicators of External Vulnerability, 1997-20031/

(In percent of GDP unless otherwise specified)

1997

1997

1998

998
2000
2001

2002

2003

proj.

Financial Indieators

Public sector debt

Buad moncy (percent change, 12-ruonth basis)

Credit to non-government (percent change, 12-month basis) 2/

Real wedit to non-government (percent change, 12-month basis) 2/

Motsthly weighted average $\mathbf{T}$-bill rate

Monthly weighted awerage real T-bill rate $3 /$

28.1
104.8
33.7
-46.9
133.5
-7.1

27.6

$27.6 \quad 30.3$

$48.9 \quad 44.9$

$64.7 \quad 45.8$

17.0

82.4

29.7

$-9.2$

105.2

32.6

30.0

38.0

50.5

7.4

62.4

15.4

27.7

46.2

59.9

26.0

38.4

6.3

25.7

38.1

46.3

29.6

17.4

$-0.3$

20.1

35.5

35.0

20.7

158

$-0.7$

External Indicators

Expots (percent charge, 12-month basis in USS)

Imports (percent change, 12-month basis in US\$)

Terms of Trade (percent change, 12-month basis)

Current account balance

Current account balance after FDI

Errors and omissions

Gross official reserves (in US\$ nillions)

(in months of imports GNFS of the following year)

Central Bank short-term foreign liabilities (in US\$ millions)

Gross reserves of the banking system (in US\$ millious)

(in months of imports GNFS of the following year)

Short term foreign liabilities of the commercial banks (in USS)

Open foreign currency position of the commercial banks (in US\$)

Official reserves/Brond money (M2)

Official Jeserves/Narrow money (M0) $6 /$

Total short tern extemal debt by remaining maturity $/$

$$
\text { In percent of reserves }
$$

ln percent of total debt

Total external debt (in US\$ millions)

Of which: Public and Publicly guaranteed debt

Total external debt (in percent of exports of $G \& S$ )

Total external debt/ GDP

External interest payments (in percent of exports of G\&S)

External amortization payments (in pereent of exports of G\&S)

Exchange rate (per US\$, period average)

REER appreciation $(+)$ (12-month basis)

\section{Financial Market Indicators}

Foreign currency debt ratings

Moody's

Standard and Poor's

Spread of benchmark bonds (basis points, end of period)

\begin{tabular}{rrrrrrr}
4.6 & -1.6 & 2.4 & 21.9 & 9.8 & 21.8 & 9.2 \\
-1.4 & 4.8 & -12.1 & 23.7 & 19.1 & 14.8 & 13.3 \\
9.0 & -4.0 & 0.2 & 2.7 & 2.3 & 1.6 & -4.2 \\
-5.4 & -6.9 & -4.0 & -3.9 & -5.5 & -3.4 & -4.5 \\
-1.9 & -2.0 & $-1,2$ & -0.9 & -2.4 & -0.9 & -1.6 \\
3.2 & 1.4 & 2.3 & 0.9 & 2.4 & -2.0 & 0.0 \\
3,075 & 2,278 & 2,462 & 3,466 & 5,090 & 6,975 & 8,041 \\
2.9 & 2.4 & 2.1 & 2.5 & 3.2 & 3.9 & 4.2 \\
100 & 0 & 170 & 100 & 100 & 0 & 0 \\
4,763 & 3,768 & 3,623 & 4,963 & 6,686 & 8,138 & 9,204 \\
4.5 & 3.9 & 3.1 & 3.6 & 4.3 & 4.6 & 4.8 \\
267 & 188 & 221 & 225 & 371 & 491 & 520 \\
1,400 & 1,933 & 724 & 318 & 353 & 380 & 423 \\
40.3 & 27.0 & 33.5 & 48.6 & 59.5 & 62.5 & 58.8 \\
242.6 & 106.1 & 143.6 & 187.5 & 241.7 & 302.3 & 232.6 \\
8.0 & 6.5 & 4.7 & 5.3 & 6.7 & 8.6 & 9.1 \\
91.8 & 119.8 & 68.1 & 56.5 & 52.6 & 56.5 & 59.9 \\
27.4 & 29.6 & 23.8 & 19.1 & 22.8 & 28.4 & 30.6 \\
9,467 & 9,903 & 8,771 & 10,273 & 11,742 & 13,887 & 15,765 \\
6,854 & 6,967 & 6,169 & 6,955 & 7,716 & 8,920 & 9.847 \\
95.1 & 104.0 & 88.9 & 84.7 & 87.5 & 85.7 & 89.3 \\
26.9 & 23.5 & 24.5 & 27.7 & 29.2 & 30.4 & 29.9 \\
5.0 & 5.7 & 5.1 & 4.4 & 4.6 & 3.9 & 4.4 \\
15.9 & 18.0 & 23.9 & 12.5 & 14.5 & 14.4 & 16.3 \\
7.195 & 8,881 & 15,274 & 21,688 & 29,056 & 33,106 & $\ldots$ \\
16.5 & 30.0 & -14.8 & 9.5 & 1.5 & 2.7 & $\ldots$ \\
& & & & & & \\
& & & & & & \\
$\mathrm{BA3}$ & $\mathrm{B} 3$ & $\mathrm{~B} 3$ & $\mathrm{~B} 3$ & $\mathrm{~B} 2$ & $\mathrm{~B} 1$ & $\mathrm{~B} 1$ \\
$\mathrm{BB}-$ & $\mathrm{B}-$ & $\mathrm{B}-$ & $\mathrm{B}-$ & $\mathrm{B}$ & $\mathrm{B}+$ & $\mathrm{BB}$ \\
350 & 1,300 & 780 & 406 & 421 & 282 & 279 \\
& & & & & & \\
\hline
\end{tabular}

Sources: Romanian authorities; and Fund staff estimales.

I/ All stocks are measured end-of-period.

2/ Adjusted for bad loans written oft. Real credit growth is weighted average of real lei credit growth, and U.S. doliar-measured foreign currency growth.

3. The real rate is based on the ex-post 12-month CPI inflation.

4/ Actual as of January 2003.

5/ Actual as of January 2003.

6. Narrow moncy is defined as currency plus lei-denominated sight dcposits.

7/ Defincd as short-tcm debt by original maturity basis plus amortization falling due on medium-term loans and bonds.

$8 /$ Actual on March 14,2003.

9/ In 2001 the benchmark bond is the '05, while for carlier ycars it is the '02, Actual on March 19, 2003.

10/ On-balance-sheet only. The orerall open currency position, including off-balance-sheet items and capital paid in foreign exchange, was US\$11 1 million as of end-December. 
Table 9. Romania: Indicators of Fund Credit, 2000-07

(In percent, unless otherwise indicated)

\begin{tabular}{|c|c|c|c|c|c|c|c|c|}
\hline & 2000 & 2001 & 2002 & 2003 & 2004 & 2005 & 2006 & 2007 \\
\hline \multicolumn{9}{|l|}{$\begin{array}{l}\text { Outstanding Fund Credit } \\
\text { (end of period) }\end{array}$} \\
\hline In millions of SDRs & 347.9 & 308.0 & 314.9 & 400.7 & 321.7 & 263.7 & 161.9 & 48.2 \\
\hline In millions of U.S. dollars & 458.8 & 397.9 & 401.2 & 514.1 & 412.7 & 338.3 & 207.7 & 61.8 \\
\hline In percent of quota & 33.8 & 29.9 & 30.6 & 38.9 & 31.2 & 25.6 & 15.7 & 4.7 \\
\hline In percent of GDP & 1.2 & 1.0 & 0.9 & 1.0 & 0.7 & 0.5 & 0.3 & 0.1 \\
\hline In percent of exports of goods and services & 3.8 & 3.0 & 2.5 & 2.9 & 2.2 & 1.6 & 0.9 & 0.3 \\
\hline In percent of official reserves & 13.2 & 7.8 & 5.8 & 6.4 & 4.7 & 3.5 & 2.0 & 0.5 \\
\hline \multicolumn{9}{|l|}{ Debt service due to the Fund } \\
\hline (in millions of U.S. dollars) & 120.3 & 118.7 & 109.5 & 115.9 & 114.6 & 85.4 & 139.7 & 151.5 \\
\hline In percent of quota & 11.7 & 11.5 & 10.6 & 11.2 & 11.1 & 8.3 & 13.6 & 14.7 \\
\hline In percent of GDP & 0.3 & 0.3 & 0.2 & 0.2 & 0.2 & 0.1 & 0.2 & 0.2 \\
\hline In percent of exports of goods and services & 1.0 & 0.9 & 0.7 & 0.7 & 0.6 & 0.4 & 0.6 & 0.6 \\
\hline In percent of official reserves & 3.5 & 2.3 & 1.6 & 1.4 & 1.3 & 0.9 & 1.3 & 1.3 \\
\hline
\end{tabular}

Sources: Treasurer's Department; Romanian authorities; and Fund staff calculations. 
Table 10. Romania: Medium-Term Projections, 2000-07

\begin{tabular}{|c|c|c|c|c|c|c|c|c|}
\hline & 2000 & 2001 & 2002 & 2003 & 2004 & 2005 & 2006 & 2007 \\
\hline & & & & \multicolumn{5}{|c|}{ Projected } \\
\hline \multicolumn{9}{|l|}{ Income and prices } \\
\hline Nominal GDP (in trillions of lei) & 803.8 & $1,167.2$ & $1,512.3$ & 1.838 .7 & $2,155,4$ & $2,437.8$ & $2,698.8$ & $2,953.8$ \\
\hline Nominal GDP (in billions of US dollars) & 37.1 & 40.2 & 45.7 & 52.7 & 56.7 & 52.0 & 67.7 & 73.8 \\
\hline Real GDP & 2.1 & 5.7 & 4.9 & 4.9 & 5.0 & 5.0 & 5.0 & 4.8 \\
\hline Domestic Demand & 4.3 & 8.2 & 3.9 & 5.8 & 5.1 & 5.0 & 5.0 & 4.8 \\
\hline Household consumption & -0.8 & 6.3 & 3.2 & 4.8 & 4.2 & 4.0 & 4.0 & 3.8 \\
\hline Government consumplion & 12.3 & 4.7 & 2.1 & 3.6 & 1.1 & 2.9 & 2.9 & 2.7 \\
\hline Gross fixed investment & 5.5 & $9 !$ & 8.3 & 10.7 & 10.6 & 10.8 & 10.5 & 8.8 \\
\hline of which: non govemment & 5.2 & 9.6 & 8.4 & 9.5 & 11.0 & 11.0 & 10.5 & 9.0 \\
\hline of which: govermment & 9.4 & 3.6 & 7.6 & 25.0 & 6.8 & 8.3 & 11.0 & 8.0 \\
\hline Increase in stocks (contribution) 1 / & 2.2 & 1.8 & 0.0 & 0.3 & 0.3 & 0.0 & -0.1 & 0.0 \\
\hline External Demand (contribution) & .2 .3 & -3.0 & 0.7 & -1.5 & -0.6 & .0 .5 & -0.5 & $=0.5$ \\
\hline Exports of goods and services & 23.4 & 11.1 & 16.9 & 11,2 & 7.5 & 6.5 & 6.5 & 6.5 \\
\hline Imports of goods and services & 27.1 & 17.2 & 12.1 & 12.2 & 7.2 & 6.2 & 6.2 & 6.2 \\
\hline GDP deflator, p.a. & 44.2 & 37.3 & 23.6 & 15.9 & 11.6 & 7.7 & 5.4 & 4.4 \\
\hline CPI, e.o.p. & 40.7 & 30.3 & 17.8 & 14.0 & 10.0 & 6.0 & 5.0 & 4.0 \\
\hline CPI, p.д. & 45.7 & 34.5 & 22.5 & 16.2 & 11.6 & 7.7 & 5.4 & 4.4 \\
\hline \multicolumn{9}{|l|}{ Saving and investment balances } \\
\hline Total domestic saying & 14.2 & 14.9 & 17.3 & 16.9 & 17.9 & 18.6 & 19.2 & 19.5 \\
\hline Net factor rexeipts and transfers from abroad & 1.4 & 2.1 & 2.3 & 2.4 & 2.4 & 2.3 & 2.3 & 2.2 \\
\hline Total pational saving & 15.6 & 17.0 & 19.7 & 19.3 & 20.3 & 20.9 & 21.5 & 21.7 \\
\hline Non-government & 16.6 & 17.1 & 19.1 & 18.3 & 19.0 & 39.3 & 19.5 & 19.7 \\
\hline of which: energy sector. & 1.2 & 1.2 & 2.3 & 2.8 & 3.2 & 3.3 & 3.3 & 3.3 \\
\hline Government & $-1,0$ & -0.1 & 0.6 & 1.1 & 1.3 & 1.6 & 1.9 & 2.0 \\
\hline Total investment & 19.5 & 22.6 & 23.1 & 23.8 & 24.8 & 25.4 & 25.9 & 26.0 \\
\hline Non-govemment & 16.4 & 19.4 & 19.9 & 20.0 & 21.0 & 21.5 & 21.8 & 22.0 \\
\hline of which: energy sector & 2.7 & 2.8 & 3.0 & 3.2 & 3.2 & 3.2 & 2.8 & 2.8 \\
\hline Government & 3.0 & 3.1 & 3.2 & 3.7 & 3.8 & 3.9 & 4.1 & 4.0 \\
\hline Savings - investment balance & -3.9 & -5.5 & -3.4 & -4.5 & -4.5 & -4.5 & -4.4 & .4 .3 \\
\hline Non-govemment & 0.1 & -2.3 & -0.8 & -1.8 & $-2,0$ & -2.2 & -2.3 & .2 .3 \\
\hline of which: energy sector & -1.5 & -1.6 & -0.7 & -0.4 & 0.0 & 0.1 & 0.5 & 0.5 \\
\hline Govemment & -4.0 & -3.2 & -2.6 & -2.7 & -2.5 & -2.3 & -2.2 & $-2,0$ \\
\hline
\end{tabular}

Sources: Romanjan authorities; and Fund staff estimates and projections.

L/Includes statistical discrepancy. 
ROMANIA--RELATIONS WITH THE FUND

(As of February 28, 2003)

I. Membership Status: Joined 12/15/72; Article VIII

II. General Resources Account:

SDR million

$1,030.20$

$1,338.50$

Fund holdings of currency

III. SDR Department:

Net cumulative allocation

Holdings

IV. Outstanding Purchases and Loans:

Stand-By Arrangements

Systemic Transformation
SDR million

75.95

4.25

SDR million

261.17

47.13
$\%$ Quota

100.00

129.93

$\%$ Allocation 100.00

5.59

\% Quota

25.35

4.57

V. Financial Arrangements:

Type

\begin{tabular}{|c|c|c|c|}
\hline & $\begin{array}{l}\text { Expira- } \\
\text { tion }\end{array}$ & $\begin{array}{l}\text { Amount } \\
\text { Approved }\end{array}$ & $\begin{array}{l}\text { Amount } \\
\text { Drawn }\end{array}$ \\
\hline Da & Date & (SDR million) & (SDR million) \\
\hline
\end{tabular}

Stand-By

$10 / 31 / 01 \quad 04 / 29 / 03$

300.00

134.67

Stand-By

$08 / 05 / 99 \quad 02 / 28 / 01$

400.00

139.75

Stand-By

$04 / 22 / 97 \quad 05 / 21 / 98$

301.50

120.60

Stand-By

$05 / 11 / 94 \quad 04 / 22 / 97$

320.50

94.27

Stand-By

$05 / 29 / 92 \quad 03 / 28 / 93$

314.04

261.70

Stand-By

$04 / 11 / 91 \quad 04 / 10 / 92$

380.50

318.10

VI. Projected Payments to Fund (Expectations Basis) ${ }^{1}$

(SDR million; based on existing use of resources and present holdings of SDRs):

\begin{tabular}{lrrrrr} 
& \multicolumn{6}{c}{ Forthcoming } \\
\cline { 2 - 6 } & $\underline{2003}$ & $\underline{2004}$ & $\underline{2005}$ & $\underline{2006}$ & $\underline{2007}$ \\
Principal & 72.98 & 115.29 & 89.02 & 31.00 & \\
Charges/interest & $\underline{6.23}$ & $\underline{6.09}$ & $\underline{3.43}$ & $\underline{1.76}$ & $\underline{1.31}$ \\
Total & $\underline{79.21}$ & $\underline{121.38}$ & $\underline{92.45}$ & $\underline{32.76}$ & $\underline{1.31}$
\end{tabular}

\footnotetext{
${ }^{1}$ This schedule presents all currently scheduled payments to the IMF, including repayment expectations where applicable and repayment obligations otherwise. The IMF Executive Board can extend repayment expectations (within predetermined limits) upon request by the debtor country if its external payments position is not strong enough to meet the expectations without undue hardship or risk.
} 


\section{Exchange Rate Arrangements}

From August 1994, a decentralized direct dealing interbank market has been in operation. The official reference rate published by the NBR is an average of rates reported for interbank and client transactions. Romania has accepted the obligations of Article VIII, Sections 2, 3, and 4, with effect from March 25, 1998. Romania's exchange system is free of restrictions on the making of payments and transfers for current international transactions. Romania maintains de jure a managed floating exchange rate regime with no preannounced exchange rate path, while the de facto regime is an unannounced crawling band.

\section{Article IV Consultation}

Romania is on the 24-month consultation cycle subject to the provisions of the decision on consultation cycles approved by the Board on July 15, 2002. The last consultation was concluded on January 8, 2003.

\section{Technical Assistance}

The transition in Romania has been supported by substantial technical assistance from multilateral agencies and bilateral donors. The Fund has provided support in a number of areas with more than 40 technical assistance missions since 1990, although the authorities have had a mixed record with regard to implementation. Expert Fund assistance has focused on a number of key areas, including: fiscal reforms; modernization of the central bank and the banking system; creating a market-oriented legal structure; training; and improving the collection and reporting of statistics. The implementation of a comprebensive tax administration reform designed in line with the recommendations of several technical assistance missions of the Fund's Fiscal Affairs Department has started in January 2003. A report on the observance of standards and codes (ROSC) on fiscal transparency was completed on November 6, 2002 (IMF Country Report No. 02/254).

\section{Safeguards Assessment}

Under the Fund's safeguards assessment policy, National Bank of Romania (NBR) is subject to an assessment with respect to the Stand-By- Arrangement, which was approved on October 31, 2001. A full safeguards assessment of the National Bank of Romania (NBR) was completed on May 13, 2002. The assessment identified certain weaknesses and made appropriate recommendations, as reported in $\mathrm{EBS} / 02 / 150$, which the authorities have begun to implement. The two high-priority recommendations - a quarterly reconcillation between the NBR's accounting records and economic data, submitted to the Fund, and implementing the bank's internal regulation regarding controls over foreign reserve management - have already been implemented. 
XI. Resident Representative

The Fund has had a resident representative in Bucharest since 1991. Mr. Graeme Justice assumed the post in January 2003. 


\section{ROMANIA: RELATIONS WITH THE WORLD BANK}

\section{Partnership in Romania's Development Strategy}

1. The Romanian authorities are pursuing a strategy designed to maintain macroeconomic stability and establish sustainable economic growth and poverty reduction, moving towards the eventual goal of accession to the European Union. Key elements of this policy include tightening payments discipline throughout the economy, especially in the energy sector, hardening budget constraints for state-owned enterprises, actively pursuing enterprise and banking privatization and restructuring, and strengthening the social safety net. Over the medium term, continuation of the current economic recovery will depend critically on the maintenance of reform momentum and moving steadily ahead with governance and private sector development reforms. Such reforms would stress transparency and accountability in government, improvements in the business environment, and actions to strengthen the regulation and oversight of financial and capital markets.

2. In broad terms, the IMF has taken the lead in assisting Romania in maintaining macroeconomic stability. As such its program focus is on the fiscal framework, monetary policy and those elements of the structural reform program that have a direct bearing on the macroeconomic situation. The main vehicle for its dialogue with the authorities is an 18-month Stand-By Arrangement (SBA) covering the period October 2001 to March 2003. The First and Second Reviews under the SBA were completed successfully in August 2002, while the conclusion of the Third Review is expected to be considered by the IMF Board in April 2003.

3. The World Bank has taken the lead in policy dialogue on structural issues, including poverty reduction measures, private sector development, institution building and governance. It has a range of instruments through which it conducts dialogue, including adjustment lending operations such as PSAL 1 and PSAL 2, a planned series of Programmatic Adjustment Loans, several sector investment operations and economic work such as the recently completed Public Expenditure and Institutions Review (PEIR), and a planned Country Economic Memorandum (CEM).

4. In some areas where the Bank takes the lead in the dialogue, its analysis is shared with the IMF and is used as input for the fiscal framework, but there are no directly corresponding structural benchmarks or policy conditions in the IMF SBA. In other areas where the Bank takes the lead, the SBA does reflect certain elements in its conditionality. These are generally structural reform measures which have important fiscal implications, such as energy sector reform and restructuring. There are further areas of formal joint responsibility such as the Financial Sector Assessment Program (FSAP).

5. Overall the IMF and World Bank staff maintain a close collaborative relationship in supporting the Government's reform program and are coordinating their policy advice to the Romanian authorities. 


\section{IMF-World Bank Collaboration in Specific Areas}

\section{Areas in which the World Bank leads and there is no direct IMF involvement}

6. The policy areas in which the Bank leads and which are not directly incorporated into the IMF program or country dialogue include strengthening the social safety net, revitalizing the economy in rural areas, institution building and governance, and improving the business environment. Bank policy dialogue for strengthening the social safety net includes poverty monitoring, social assistance, pension reform, child welfare reform, health sector reforms, and labor retraining and redeployment. These have been supported by the Bank's Employment and Social Protection Loan, the Social Sector Development Loan, the Social Development Fund and the Mine Closure Loan. They are further supported by economic work such as the recently completed Poverty Profile and the Local Services Delivery Study, and by the planned Poverty Assessment, Pension Reform Study, and Gender Assessment.

7. The Bank has a range of programs to revitalize the economy in rural areas. These include the Rural Finance Loan which supports income generation activities, complemented by a Rural/Urban Linkage Growth Study and a Rural Development Loan supporting infrastructure development and improved governance at the community level. The Irrigation Rehabilitation loan supports infrastructure rehabilitation and institutional reform. Other supporting economic work includes the Community Networks Study and the Jiu Valley Development Study.

8. The Bank has a strong institution building and governance program. This builds on recent reforms in local public finance and taxation and decentralization. Policy dialogue has been facilitated the recently completed Public Expenditure and Institutions Review (PEIR) which included analysis and recommendations relating to 1) improved resource allocation in the health sector; 2) delivery of social assistance at the local level; 3) pension reform; 4) institutions for budget management 5) fiscal decentralization; and 6) reforms to the budget process in the judiciary. The policy dialogue will continue through a Country Financial Accountability Assessment (CFAA) now underway. The Bank completed a Diagnostic Survey of Corruption in Romania prior to the Government's preparing its National Program for Prevention of Corruption. Work is now beginning on a series of Programmatic Adjustment Loans (PALs) the first of which will focus on institutional and governance reforms in the civil service, the judiciary and public expenditure management, as well as support for private sector development and the business environment and improvements in the delivery of public services. This will be supported by a $\$ 18.6$ million Private and Public Sector Institution Building Loan (PPIBL) which will provide technical assistance for these activities and others relating to privatization. Other institution-building support includes financing of a cadastre and land titling project, a project on the environmental and social aspects of mine closures, a hazards mitigation project cofinanced by a GEF grant which focuses on reducing damage from earthquakes, floods, toxic wastes and other hazards in high risk areas, and a regional project on Trade and Transportation Facilitation in South East Europe.

9. Policy advice and support for reforms in the business environment have been offered by the Bank in several areas. Through PSAL 1 and PSAL 2 the Bank has focused on 
reducing barriers to entry for firms and joint ventures, implementing internationally recognized accounting standards and introducing improved banknuptcy and liquidation procedures. The PALs will also address business environment issues, and all these initiatives will be supported by technical assistance provided under the PPIBL.

\section{Areas in which the World Bank leads and its analysis serves as input into the IMF program}

10. The Bank supports the GOR's privatization program through PSAL 2 which includes targets and timelines for privatization of commercial SOEs and financial institutions. The program builds on the progress established under PSAL 1 and identifies pools of SOEs from which set minimum numbers of enterprises are selected for case-by-case privatization and workout. Specific enterprises including Alro, Alprom, and Petrom, were also identified. Key to the success of this program is agreement with the Bank on strategies for the privatization of theses entities and the subsequent adoption of a clear, open and transparent tender process using the services of qualified investment advisors in a manner consistent with Bank policies.

11. The Bank has led the dialogue on restructuring, reform and eventual privatization of enterprises in the electricity, district heating and gas industries and on the adoption of regulatory legislation in preparation for privatization to strategic investors. As part of this process the Bank is supporting, inter alia, energy tariff reforms and the elimination of arrears in the electricity, district heat and gas sectors, and these objectives are woven into the policy conditions of PSAL 2, along with conditions related to the privatization of electricity distribution enterprises. These conditions have been closely coordinated with the IMF since the SBA also includes provisions for structural reforms in the energy sector including tariff setting, arrears clearance and collection rates, as well as one industry structure condition requiring the shift of seven heat-producing units to local municipalities. The Bank is leading the IFI dialogue with the Government on such critical long-term issues as power generation sector restructuring and the integration of the Romanian power sector with the South East European Regional Electricity Market and with the EU's electricity market, and provides assistance on the associated regulatory and market operation issues. The Bank is also providing technical assistance to the Ministry of Industry to establish a system of pricing and taxation of gas consistent with attracting further investment into the industry. Policy dialogue in these and other areas, notably including the district heating sector, will be further facilitated by ongoing dialogue with the Government on the recently completed Energy and Infrastructure Strategy paper and its operational recommendations. Again, the series of PALs will be used as vehicles for incorporating policy advice in these areas into Bank lending operations.

12. The Bank has taken the lead in dialogue and policy advice regarding regulation, restructuring, privatization and liquidation of banks. Under PSAL 1 the Law on Bank Privatization was modified and transparent privatization procedures established. Liquidity and solvency requirements of the NBR were tightened and provisions introduced for improved compliance with prudential regulations and external audits conducted according to International Accounting Standards. Legal and institutional reforms were introduced for orderly disposition of non-performing assets. These policy changes underpinned liquidation of the most troubled bank (Bancorex) while Bank Agricola was restructured and eventually 
privatized as were two smaller state owned banks. This policy agenda is continued under PSAL 2 with steps to complete the sale of Banca Comerciala Romana (BCR) and to restructure the Savings Bank (CEC). These initiatives have been supported by the IMF which has included Stand-By performance criteria relating to privatization of BCR and prior actions related to NBR provisioning requirements. The Bank is continuing dialogue with the government on the need to move forward with further improvements of the legal and regulatory framework for the securities and insurance sectors as well as the banking sector. The PPIBL will provide funding for these activities.

\section{Areas of shared responsibility}

13. While the Bank has taken the lead in privatization and in structural reforms in the energy sector as described above, the IMF has a strong interest from a macroeconomic perspective since privatization revenues are used to retire public debt, and quasi-fiscal deficits of the SOEs present a threat to macroeconomic stability. Accordingly, there is a high degree of consultation and coordination between the two institutions on these matters. The IMF has introduced into its SBA conditions relating to electricity tariff rates, arrears in gas and electricity collections as well as the privatization of a subset of the enterprises covered under the Bank's program. While the terms of the respective programs are mutually consistent and supportive, the focus and motivation for the Bank's program are on the efficiency of the resulting market structures, improved enterprise operation, service delivery and financial performance, and the transparency of the process and improvement in overall sector governance, and that of the IMF are on immediate revenue generation and expenditure control, and containment of ongoing fiscal burdens.

14. The Bank and the IMF have a formal agreement to conduct jointly a Financial Sector Assessment Program on which work will be completed this fiscal year.

\section{Areas in which the IMF leads and its analysis serves as imput into the World Bank program}

15. While the Bank has focused on simplifications to the taxation system as a means of improving the business environment, the Fund leads the dialogue on fiscal matters. In addition to the achievement of overall fiscal targets, the Fund's Stand-By program includes structural benchmarks and performance criteria relating to introduction of new VAT and profits tax laws, increases in specific excises, and elimination of tax preferences under the custorns duty and profit tax systems. The IMF is also providing technical assistance in support of unification of the payroll tax systems.

16. The IMF leads the dialogue on certain polices to contain certain expenditures in the public sector. These include policies regarding wage-setting in both the public service and SOEs. The IMF requires monitoring reports of the budgets of a set list of SOEs and has conditions in its Stand-By program relating to the containment of the wage bill in these enterprises and to the achievement of agreed layoff targets. In addition, the IMF leads the discussions relating to requirements for disconnections of nonpayers in the gas and electricity sectors. 
17. In these areas the Bank takes into account the policy recommendations of the IMF and ensures that its own policy advice is consistent.

\section{Areas in which the IMF leads and there is no direct World Bank involvement}

18. The IMF is fully responsible for the dialogue on monetary policy, interest rates, the exchange rate regime, the balance of payments and all the related statistical and measurement issues.

\section{World Bank Group Strategy and Lending Operations}

19. A Country Assistance Strategy (CAS) for Romania was presented to the World Bank's Board on June 19,2001. The CAS defines the Bank's lending and advisory services based on the Government's priorities of poverty reduction and EU accession, and builds on the work initiated under the Comprehensive Development Framework, for which Romania was one of the pilot countries. About one half of the FY02-04 lending program is adjustment lending. Two distinct lending scenarios were outlined. A low case in which Romania pursued a hesitant pace of reforms would result in significant reductions in Bank Group support to a level of $\$ 60$ million during FY02-04 with actions centered on a few poverty focused interventions. However, Romania has successfully remained in the high-case lending scenario under which Bank commitments of up to \$995 million during FY02-04 were envisaged, compared with $\$ 600$ million in FY98-00. This reflects the fulfillment of the highcase triggers, which included progress in establishing a macroeconomic environment conducive to lower inflation and sustainable growth as well as progress on structural issues including privatization as initiated under PSAL 1, initiation of the privatization and work-out process for a number of remaining large state-owned enterprises, agreement on a plan and schedule for reducing energy arrears, agreement on a method and timetable for privatization of Romanian Commercial Bank and the Savings Bank, improvements in the financial sector legal and supervisory framework, an agreement on an anti-corruption strategy, and approval of social assistance legislation.

20. The high-case scenario embodies a program of accelerated structural and sectoral reforms that will pave the way for Romania's eventual accession to the EU. The first stage of this program has now been launched with the approval of PSAL 2 by the Bank Board in September 2002, and the delivery of the Public Expenditure and Institutions Review (PEIR) in July 2002. In the second stage to follow PSAL 2, the Bank would offer support through a series of Programmatic Adjustment Loans (PALs) focusing on institutional and governance reforms, completion of the privatization agenda, implementation of the recommendations of the Energy and Infrastructure Strategy Paper and improvements to the business environment, as well as a Country Financial and Accountability Assessment.

21. The World Bank has been active in Romania since 1991 and has built up a portfolio of 36 Bank-financed operations with total commitments of US $\$ 3.7$ billion. Romania's portfolio is the second largest in ECA in terms of number of projects and the third largest in terms of commitments. The active portfolio as of March 12, 2003 consists of 22 projects with aggregate net commitments totaling $\$ 1,376$. 1 million and a total undisbursed balance of 
$\$ 762$ million. Sector composition in terms of net commitments is dominated by energy and infrastructure ( 58 percent, seven projects) and the social sectors ( 28 percent, also seven projects).

22. The quality of the Bank's Romania portfolio has been improving over the past several years. Since the previous Portfolio Review, many of the generic issues identified have been addressed. Specifically:

23. Project management capacity has improved in most implementing agencies. Concerns are still related to the: cumbersome bureaucratic procedures that have to be faced by the Project Implementation Units (PIUs); limited coordination capacity among key Governmental agencies; proper staffing and office location and facilities of some PIUs; motivation and retaining of experienced and qualified staff.

24. The most significant financial management issues signaled during the previous Portfolio Review, namely the imposition of ceilings to withdrawals from external financing and the timely availability of counterpart contribution, seem to have been addressed for most of the projects. This was done, however, on a case by case basis. Concerns remain as the scarcity of human and financial resources may provoke delays in project implementation and adversely affect the projects in advanced stages of preparation and those presented to Board after the approval of the State Budget.

25. IFC has dedicated substantial resources to implementing a strategy intended to advance structural reform, assist the development of capital markets and infrastructure (utilities and telecommunication), and provide support for small and medium enterprises (SMEs). To date, IFC has approved and disbursed over US\$185 million, in 22 projects, including a loan of US\$75 million for bank privatization earlier this year. In addition to investments, IFC has undertaken a number of advisory assignments aimed at supporting the privatization and restructuring of large state-owned enterprises, public utilities and the health sector. The degree of IFC's future success will be dependent to an extent on the Government's willingness to demonstrate a sustained commitment to accelerated reform, particularly with regard to transparency in enterprise privatization, reform of the banking and financial sector, restructuring and deregulation of the energy sector, fiscal decentralization, land privatization and continued reform of the agricultural sector, access to quality education in rural areas, and reform of the pension system to ensure sustainability in the context of an aging population. The ultimate success will also be dependent upon the ability of Romania to attract foreign direct investment.

Questions may be referred to Ron Hood (473-0115) or Myla Williams (473-6997) 


\section{RoMavia: Statistical Issues}

1. Romania's data collection and reporting is adequate for surveillance and program monitoring. The authorities have made limited progress in improving their statistical base over the past three years with the help of Fund TA missions, although more needs to be done, in particular in the areas of balance of payments and fiscal statistics, and the consistency between monetary and fiscal data. Romania has participated in the GDDS since February 2001. A STA mission visited Romania in November 2000 to assist the authorities in preparing metadata. The mission also prepared a data module for the ROSC, which is posted on the IMF website.

\section{Real Sector}

2. The national accounts statistics produced by the National Institute for Statistics (INS) for quarterly and annual series on Gross Domestic Product (GDP) are methodologically sound, but arrive with lag of about 3 months. Furthermore, harmonization of quarterly and annual national accounts is not done on a regular basis and late revisions can sometimes be significant; the 1999 GDP growth rate, previously reported as -3.2 percent, was revised to -2.3 percent in April 2001. The INS has moved to update the framework from the European System of Accounts 1979 (ESA79) to ESA95, and data based on ESA95 were released the end of 2000 . The INS staff has been downsized significantly over the past two years, which might affect the quality and timeliness of the data.

3. The Consumer Price Index (CPI) is subject to the standard annual re-weighting, and it is considered to be a reliable source. However, the INS reduced the coverage of the Producer Price Index (PPI) from the value of all shipments of the covered industries to shipments for domestic uses only, beginning with 1998 data. The INS planned to reinstate export coverage with the next index revision sometime in 2001.

\section{Public Finance}

4. Consolidated data on central government operations are yearly reported for the GFS Yearbook and quarterly reported for IFS, while the EU1 department receives monthly budget data. Transaction data, but not debt data, through 2001 are soon to be in the GFS Yearbook 2002. A multi-sector statistics mission visited Romania in December 1999 and recommended that all major fiscal categories be reported on a quarterly and monthly basis. The mission also called for the documentation and reporting of all government transactions related to the Agency for Bank Asset Recovery (AVAB), which has received large amounts of nonperforming bank claims in exchange for the bank receipts of government securities. The multi-sector mission also recommended the introduction of detailed financing and debt classifications in the Treasury's chart of accounts and budget classifications. These recommendations have not yet been implemented as of end 2002. Work continues on the establishment of a new aid coordination unit to monitor data on grants. 


\section{Monetary and Financial}

5. The National Bank of Romania (NBR) reports money and banking statistics for $I F S$ on a regular and timely basis. The NBR adopted accrual accounting as part of the introduction of a new chart of accounts. However, loans extended by the NBR and the commercial banks continue to be recorded on a cash basis. Also, in contrast to the Monetary and Financial Statistics Manual and the fifth edition of Balance of Paymenis Manual residency recommendations, NBR's foreign assets and liabilities exclude the central bank's claims on, and liabilities to, international organizations (except liabilities to the IMF), which are treated as domestic assets and liabilities. STA will consider in its RAP for FY2003 a monetary statistics TA mission request expressed by the NBR in November 2002.

\section{Balance of Payments}

6. The NBR routinely reports balance of payments statistics in a timely fashion. Data collection and compilation have improved following technical assistance. The ROSC data module of November 2001 noted that the authorities were in the process of implementing a program of improvements to the data collection and compilation systems for the balance of payments and international investment position data. It noted particular concerns with (1) the coverage of the direct investment data (stocks and flows) and related income flows and (2) the quality of data on current transfers, in particular with the general government sector. Improvements planned included the introduction of a survey of direct investment in Romania to address (1) above; improvements to the monitoring of aid flows into Romania to address (2); and an improved international transactions reporting system that would provide more detailed information to facilitate improved classification of transactions between residents and nonresidents of Romania. A technical assistance mission is scheduled to visit Romania in spring 2003 to address BOP data issues, including large errors and omissions and recording of repatriated earnings. 
Table 1: Romania: Core Statistical Indicators as of March 20, 2003

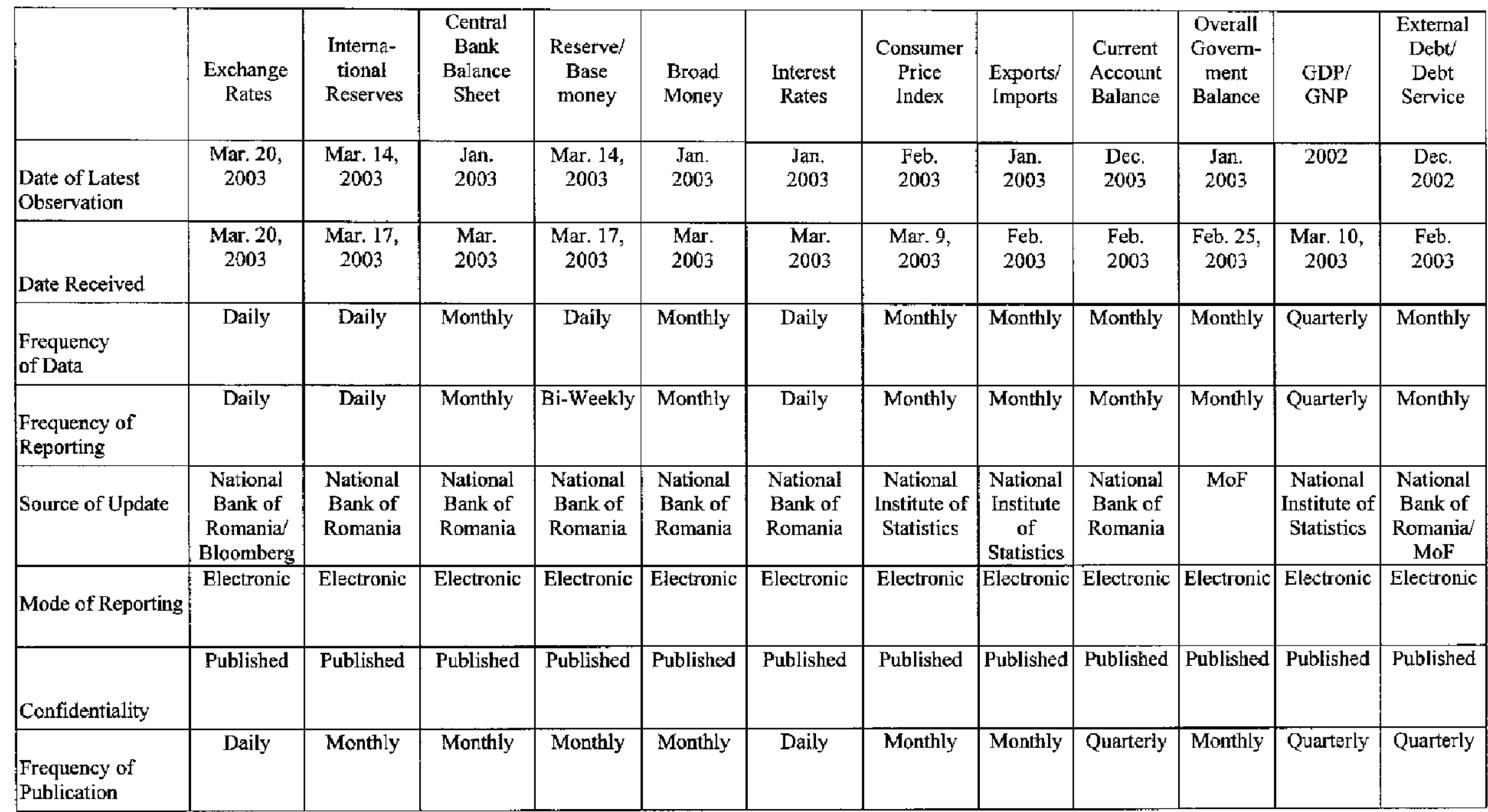


April 9, 2003

\author{
Mr. Horst Köhler \\ Managing Director \\ International Monetary Fund \\ Washington, D.C. 20431 \\ Dear Mr. Kohler:
}

In the last two years, under the economic program supported by the Stand-By Aurangement, Romania's macroeconomic performance has been very favorable. In 2002 , for a second year in a row, the economy grew by about 5 percent, inflation was reduced from 30 percent at end-2001 to 18 percent at end-2002, and the current account deficit was brought down to $3 \frac{1}{2}$ percent of GDP. Export perfornance was particularly impressive, despite sluggish growth in partner countries.

In 2003, we will continue with our policy to reduce inflation and contain the current account deficit, keeping our economy on a sustainable growth path, while also strengthening social support to segments of the population that have not yet fully benefited from recovery. Moreover, we are strongly committed to Romania's accession to the EU and to the implementation of all structural reforms necessary for completing the transition to a fully functioning market economy. This requires additional efforts to strengthen financial discipline, restructure and privatize the remaining state-owned enterprises, and complete the privatization in the banking sector. Further progress in these areas is also necessary to sustain macroeconomic stabilization and ensure that disinflation continues. For that purpose, we are requesting the completion of the third review and a $5 \frac{1 / 2}{2}$ month extension of the SBA to midOctober 2003, based on the policies presented in the attached Supplementary Memorandum on Economic and Financial Policies in 2003 (SMEFP-2). We are also requesting waivers for the nonobservance of the end-December 2002 quantitative performance criterion on the wage bill in state-owned enterprises, and the structural performance criterion on the privatization of $\mathrm{BCR}$, the largest bank in Romania. The memorandum specifies corrective measures, some of which have already been implemented, to address these issues, and other measures necessary to achieve our macroeconomic objectives. We are also requesting that our purchases be rephased, with an amount equivalent to SDR 55.111 million made available following completion of the third review and the remainder under the SBA becoming available in midAugust 2003. The attached SMEFP-2 describes the structural performance criteria, including progress in the privatization of BCR, quarterly performance criteria for a June 30, 2003 test date, benchmarks and prior actions under the program. The fourth and final review under the Stand-By Arrangement is scheduled to be completed by August 14, 2003. In light of the rephasing, a fifth review will no longer be necessary and we are requesting that it be dispensed. 
We remain determined to meet all our undertakings under the program. We believe that the policies and measures described in the attached memorandum are sufficient to achieve the objectives of the program, but we stand ready to take additional measures and seek new understandings with the IMF if necessary to keep the program on track. The Government of Romania will remain in close consultation with the IMF in accordance with the IMF's policies on such consultations, and it will provide the IMF with all the information that it requests to assess the implementation of the program.

Yours sincerely,

$$
/ \mathrm{s} /
$$

Mihai Tanasescu

Minister of Public Finance

Ministry of Public Finance /s/

Mugur Isarescu

Governor

National Bank of Romania 


\section{Supplementary Memorandum on Economic and Financial Policies in 2003}

\section{INTRODUCTION}

1. Since the completion of the first and second reviews on August 28, 2002, we have made significant progress toward realizing the main macroeconomic objectives of our program, particularly in respect to inflation, the current account deficit and growth. This memorandum updates our policies for 2003 that were originally presented in the first Supplementary Memorandum on Economic and Financial Policies (SMEFP-1), dated August 12,2002, and addresses setbacks that have recently occurred in the implementation of wage policy, enforcement of payments to energy utilities, and privatization of the remaining state-owned enterprises.

\section{Background And Request for Waivers of Performance Criteria}

2. Macroeconomic developments in $\mathbf{2 0 0 2}$ were very favorable. We estimate that GDP grew by about 5 percent, led by strong export growth that reflected the cumulative effects of private investment in consumer goods industries over the past few years. The 12-month headline inflation rate fell to $16^{\frac{1}{4}}$ percent in February, and the 2002 current account deficit was reduced much more than envisaged under the program. Strengthened confidence in macroeconomic stabilization led to rapid growth in credit, supporting recovery in investment. Foreign reserves expanded faster than programmed, and have now reached a comfortable level of $4 \frac{1}{4}$ months of prospective imports of goods and services.

\section{Monetary and budget policies have been on track. The end-December} performance criteria on NDA and NFA of the NBR were met with comfortable margins (Table 1). Somewhat faster growth in monetary aggregates reflected stronger demand for money than originally envisaged. The rapid drop in inflation initially resulted in high real interest rates, which prompted the NBR to cut its main policy interest rate by over 1,500 basis points since April 2002. The deficit target for the general government in 2002 was also met, although revenue was about $1 / 4$ percent of GDP lower than assumed in the second supplementary budget. This was offset by lower interest expenditure and payments of social benefits.

4. The financial results of the main energy utilities improved. Price adjustments in the energy sector continued as envisaged under the program. Payment enforcement measures in April and May 2002 improved the collection rates of the two gas distribution companies even more than targeted under the program, but further progress is needed in electricity and even more in heating collections. While we met our Q3 and Q4 collection targets, payments from commercial companies have slowed, which has put the March and June collection targets at risk. Particularly weak performance was reported for a group of 23 large state-owned companies slated for privatization that paid only about $3 / 4$ of their bills in Q4. Measures described in paragraphs 18 and 19 below address this issue. 
5. After encountering serious difficulties in enforcing our wage policies in Q3, we achieved a major improvement in Q4 of 2002. The end-September performance criterion on the wage bill in state-owned enterprises (SOEs) was exceeded by 4 percent as some large SOEs did not adjust their budgets after shifting large numbers of employees to newly established companies. This has also rendered the end-December performance criterion beyond reach. To meet the revised end-December target discussed with staff in October 2002 (prior action for the third review), we took decisive measures by adjusting down the budgets of these companies, and reducing by 4 percent the quarterly wage bill in all monitored SOEs. These objectives were largely achieved, and the average 2002 wage growth in the monitored group of SOEs was brought down to the economy-wide average ${ }^{1}$. Further measures in the area of wage policy are described in paragraphs 15 and 16 below. Based on this, we are requesting a waiver for the nonobservance of the endDecember performance criterion on SOEs wage bill. Our efforts to reduce employment in SOEs in 2002 did not prove very successful. Employment on a net basis was reduced by only $2 \frac{1 / 4}{4}$ percent, as about 80 percent of programmed layoffs were implemented, but new hiring exceeded the target.

6. Privatization is behind schedule, but we expect acceleration in the months ahead. We signed privatization contracts for six large companies in Q4, compared with eight set as a benchmark. We currently have seven large companies in the final negotiation phase and expect to sell all of them by end-March.

7. The relaunch of the invitation for expressions of interest in the privatization of the largest state-owned bank, BCR, proved unsuccessful. After consultation with Fund and World Bank staff, we decided to sell a blocking minority shate (25 percent plus two shares) to the EBRD and the IFC with a view to better prepare the bank for sale to a strategic investor, as described in paragraph 26 below. We therefore request a waiver for the non-observance of the end-February 2003 structural performance criterion on the completion of the BCR privatization.

\footnotetext{
${ }^{1}$ Three railway companies did not adjust their budgets after transferring employees to nonmonitored companies. As a result, the outcome for the SOEs wage budgets was 0.2 percent higher than the revised target. This small deviation does not have macroeconomic significance, but we are aware that such operations might hurt the credibility of our wage policies. The possibility to transfer employees has been eliminated under the new labor code, effective as of March 2003.
} 


\section{III.Policy Measures Agreed in the CONTEXT OF THE ThIRd ReVIew}

\section{Our main macroeconomic objective for 2003 is to consolidate stabilization,} which requires decisive progress in strengthening financial discipline in state-owned enterprises. The program envisages to: (i) reduce inflation to 14 percent by end-2003; (ii) contain the current account deficit below $4 \frac{1}{2}$ percent of GDP in 2003; and (iii) raise official reserves to a level of around $41 / 4$ months of imports by end-2003. The widening of the current account deficit relative to 2002 and the inflation target reflect the impact of the increase in the minimum wage on demand and the effects of the recent oil and gas prices shocks. We expect that GDP growth will be close to 5 percent in 2003 . We are aware that we can achieve and sustain these targets only by significantly strengthening financial discipline and accelerating privatization in the enterprise and financial sectors, areas in which the implementation of the program has so far been lagging. To ensure these results and fully complete the program supported by the SBA, we are requesting that the arrangement be extended by $5 \frac{1}{2}$ months until mid-October 2003 and purchases rephased. Quantitative performance criteria under the program for end-June 2003 are presented in Table 1, and Table 2 contains the list of prior actions, structural performance criteria and benchmarks.

\section{A. Fiscal Policy}

9. In 2003, fiscal policy will continue to support disinflation. The 2003 budget approved by parliament in November 2002 set the general government deficit target at 2.65 percent of projected GDP, $1 / 4$ percentage point below the 2002 target. In addition, we target a reduction of losses in the energy sector by $1 / 2$ percent of GDP in 2003 . The somewhat lower reduction than originally envisaged reflects a temporary increase in oil and gas prices, which we expect to be reversed toward the end of 2003. Moreover, we are committed to reduce the budget deficit target further should this prove necessary to offset excessive consumption growth that might develop if the effects of the minimum wage increase are not contained. To ensure sufficient room for such downward adjustment, we reached understandings with Fund staff on monthly revenue targets for the general consolidated budget and monthly expenditure ceilings for the state budget for the first half of 2003 (TMU; section III).

10. On revenue, the $\mathbf{2 0 0 3}$ budget marks a major step in our strategy to gradually reduce payroll taxes, complemented with measures to broaden the tax base of the pension fund:

- We cut payroll tax and social security contribution rates by 5 percentage points on January 1,2003, thereby reducing far-reaching distortions in the labor market and incentives for the growth of the informal economy. The revenue loss of about $3 / 4$ percent of GDP will be partially compensated by the full-year impact of the recently implemented VAT and profit tax laws and higher taxes on land and buildings.

- We will approve by April 20 a government decision to increase fuel excises on May 1, 2003 to restore their value in euro as of January 2003. 
- The new Labor Code, effective since March 1, 2003, no longer provides for the possibility of hiring employees on the basis of "civil contracts," most of which are exempt from pension fund contributions. To eliminate any remaining legal uncertainty, we will approve by April 10,2003 an Emergency Ordinance canceling Articles 1-7, and Articles 15, 16 (b), 18, and 20 in law 130/1999, which provided the basis for exempting "civil contracts" from the obligation to pay pension fund contributions (prior action). By July 31, 2003 we will amend the legislation on labor inspection (law 108/1999) in order to strengthen its capacity to enforce the stipulations of the Labor Code to reduce the scope of the informal economy.

- We will request by April 15, 2003, technical assistance from the World Bank, and the Fund on the issues of medium-term adjustment in gas prices and the concomitant reform in the tax regime in the gas sector (prior action). Moreover, the government will issue a decision no later than end July, thereby creating a clear pricing and taxation framework for bidders in the forthcoming privatization projects in this sector.

- We remain firmly committed to refrain from any other tax cuts in 2003. In particular, we will not introduce a reduced VAT rate or modify income tax rates.

11. On the expenditure side, the 2003 budget gives priority to increasing capital expenditure and strengthening the social safety net. Investment spending is set to increase by 0.6 percent of GDP, while total social transfers will increase by 5 percent in real terms. We have limited the increase in nominal gross wages in the budgetary sector to 6 percent in January and 9 percent in October 2003, consistent with our target for keeping the annual growth in the net wage bill in the budgetary sector at 3 percent in real terms. Moreover, while we will continue downsizing total budgetary sector employment, we will provide sufficient resources, including through the recruitment of well-qualified civil servants, for improving public administration, implementing the judicial reform, and strengthening the institutional capacity for adopting and implementing EU legislation and absorbing EU grants.

\section{In February 2003, we adopted additional measures to mitigate the social} impact of energy price increases and restructuring in state-owned companies:

- To ensure the social acceptability of reforms in the energy sector, we have adopted an improved heating support scheme for low-income households, which increased available budgetary resources by lei 1.5 trillion. We will also introduce, in two steps, the option of equal monthly payments of heating bills. Moreover, as a one-off measure to address the problem of unpaid bills of the poorest households which are beneficiaries of the minimum guaranteed income, we have allocated another lei 1.4 trillion, which will be used to reduce their arrears to local heating companies.

- To cover the additional costs inposed on the local district beating companies by mandatory equalization of charges for non-metered deliveries with the average consumption in households with meters, the regulatory agency (ANRSC) will review the need to increase the distribution tariffs by end-March. We will allocate in the 
rectified budget an amount equivalent to lei 400 billion to cover the resulting increase in subsidies.

- In the context of accelerated restructuring of companies in the process of privatization, we have allocated additional resources in an amount of lei 1,166 billion to grant to laid off employees (a) severance payments up to two times the net average economy-wide wage in January 2003; and (b) supplementary income for 20-24 months (depending on the length of service) equal to the difference between regular unemployment benefits and individual net wages during the three months preceding the layoff, under the condition that individual wages doe not exceed the net average economy-wide wage in January 2003. In the mining sector, companies that will not be able to pay severance payments out of their own budget, will also utilize this scheme.

- The above-referenced additional social measures will be financed through savings in interest expenditure (lei 1.3 trillion), the unemployment fund budget (lei 0.8 trillion), and nonpension expenditures of the pension fund (lei 4 trillion). The latter will require an amendment of law $19 / 2000$ by April 15,2003 , with a view to strengthening eligibility criteria for the two-year maternity leave through an extension of the minimum contribution period from 6 to 10 months.

13. We made important progress in implementing the tax administration reform. To improve collection efficiency, in November 2002 we approved, as a prior action, an Emergency Ordinance providing for the establishment of a common agency for collection, audit, and enforcement of social security contributions as of January 1, 2004. In January 2003, we established a separate tax administration department in the Ministry of Public Finance, appointed the responsible Secretary of State (prior action), and created a large taxpayers office for Bucharest. By end-May 2003, we will approve legislation amending the laws on the pension, unemployment and health insurance funds (in particular Law 19/2000, Law 76/2002, and Emergency Ordinance 150/2002), which will harmonize procedures for collection, enforcement and auditing (structural performance criterion). We envisage to harmonize the tax base of the social security contributions in the coming months following appropriate stadies of the impact of these changes on the various social security programs.

14. We intend to issue off-budget external guarantees of up to US\$650 million in $\mathbf{2 0 0 3}$ to facilitate investment, particularly in the railways and the second phase of the nuclear power plant Cernavoda. We consider the investment in the railway system as crucial to improving its safety and efficiency. The second phase project in Cernavoda (already at an advanced stage) is very important for our strategy to modernize Romania's energy sector. Both projects will generate resources for the repayment of the loans, and the guarantee will not impose a burden on the budget.

\section{B. Wage Policy}

15. We have approved the 2003 budgets of monitored SOEs, which limit the growth in their gross wage bill to 14 percent compared to 2002 (prior action). We are 
carefully monitoring the effects of the January minimum wage increase of 43 percent on the overall wage level. Financial plans of about 100 private and state-owned companies available in mid-February suggest that their take-home wages are set to grow by about 8 percent in real terms, and employers wage costs by 5 percent, primarily in small companies. While preliminary data available in mid-March for about 250 companies indicate a lower growth rate, more information is needed to assess the impact on the overall economy.

\section{We have decided to implement further measures to strengthen our $\mathbf{2 0 0 3}$} wage policy. Before April 15, we will issue instructions to all state-owned companies that their wage budgets will have to be adjusted for any externalization, temporary, or permanent transfer of staff to other firms. To strengthen the credibility of our wage program, and to accelerate restructuring in the over-staffed state-owned sector, the employment reduction described in paragraphs 22 and 24 will allow us to cut employment by 8,000 positions in addition to the 14,300 positions already envisaged in our 2003 wage and employment program and impose a hiring freeze in all monitored state-owned enterprises. The exceptions from this freeze will have to be approved by the Cabinet, and their total number cannot be higher than the 3,135 new positions included in the 2003 wage and employment program for January-December. To ensure that the Q1 target is met, we will block 4 percent of the Q1 wage bill in the monitored SOEs, which can only be released upon approval of the responsible Minister if the quarterly target is within reach.

\section{Energy Sector Reforms}

17. We will continue with the adjustment of administered prices (Table 3). The unified end-user price for natural gas was raised from the equivalent of US\$82.5/tcm to US\$90/tcm on March 1, 2003 (prior action), and will be further increased to US\$93/tcm on July 1, 2003 (structural performance criterion). The adjustment of the electricity prices on April 1, 2003 and July 1, 2003, as well as Termoelectrica's producer price for electricity on July 1, 2003 will maintain tariffs at their level in U.S. dollar terms at the time of the previous adjustment (structural performance criteria). Having been fixed in lei terms since July 2002, the National Reference Heating price will be raised to the equivalent of US\$20/Gcal as of July 1, 2003 (structural performance criterion).

18. To achieve the $\mathbf{2 0 0 3}$ collection targets, we will act decisively against industrial energy users with a weak payment record. The largest non-payers to the three utilities (DGN, DGS, and Electrica), as identified in Table 4, which had not fully paid by March 19, 2003, their bills (excluding penalties) for December 2002 and January 2003, were disconnected by March 25, 2003 (prior action; in the case of Siderurgica and Roman the payments were only completed on April 2). Each of these companies will stay disconnected until all bills from December 2002 onwards have been paid in full to the respective energy supplier (continuous structural benchmark). In cases where such a measure would have had unacceptable social consequences, the general consolidated budget paid the difference between amounts actually paid and total bills for December up to an amount of lei 211 billion. Starting with the bills for January 2003, if these companies do not pay in full, payments from the budget will not be available. However, 
given that we do not expect Siderurgica and the three mining companies to be able to fully pay their bills even in the coming months, the budget will provide additional support on an exceptional basis in an amount of up to lei 320 billion until June 30, 2003, by which time the restructuring measures described in paragraphs 22 and 24 will have led to their improved financial performance. We will also accelerate restructuring, including by labor retrenchment, and privatization of 22 companies in the APAPS portfolio, as described in paragraph 24 . To provide Electrica with a strong incentive to persevere in improving collections, the June 2003 indicative target on its collection rate bas been converted into a performance criterion.

19. To prevent moral hazard and strengthen financial discipline, we amended in March Emergency Ordinance 208 (prior action), which has provided for an openended cancellation of arrears owed by companies under privatization to utilities and the budget. In particular, the amendment:

- removed the reference to the cancellation and rescheduling of arrears in 23 companies accumulated after January 1,2003 in paragraph 12 (a) and (c) of the ordinance, limiting the cancellation of arrears to the stock on December 31, 2002;

- eliminated paragraph 14 , which transfers the loss resulting from the cancellation of arrears to utilities to the budget via a triangular offset;

- deleted paragraph 5, thereby limiting the write-off of accrued penalties for arrears envisaged in Article $18 \mathrm{c.}$ ) (1) in law 137/2002 to the stock on December 31, 2001.

Moreover, we will not extend the special regime provided for in paragraph 2, which protects companies under privatization from creditors, beyond six months.

20. To speed up collections from households, we have reduced the deadline for payments to 15 working days, and for disconnections to $\mathbf{4 5}$ days. In addition, all subsidies to district heating companies from the state and local budgets will be paid fully and on time into the escrow accounts. At the same time, heating distributors with arrears as of April 1 will be again disconnected from heat and gas deliveries used to supply hot water until full payment, in line with the action plan adopted in consultation with the World Bank in spring 2002.

21. Despite delays in preparatory steps, privatization projects in the energy sector will be completed on time. We have signed the contract with the privatization advisor for Petrom (prior action) and will approve the privatization strategy agreed with the World Bank by end-May 2003, with a five-month delay relative to the original schedule. We will prepare the information memorandum and launch the tender for expressions of interest by end-June (structural benchmark). For the two electricity distribution companies, we announced the expression-of-interest tenders to acquire a majority holding of at least 51 percent (prior action) on January 8, have shortlisted bidders by end-March and will invite binding offers by end-May. We signed the contract with the privatization advisors for the two gas companies in early March 2003, and will approve the privatization strategy by end-April and announce the privatization tender by 
June 30. To facilitate faster privatization, we will closely coordinate the restructuring of the electricity sector with the World Bank.

22. Restructuring of the mining sector will be accelerated. With the help of a World Bank-supported program, we will continue to downsize unviable mines. In 2003, employment in the sector will be reduced by 6,000 positions, of which 1,300 have already been cut.

\section{Privatization and Liquidation}

\section{Rapid growth and lasting macroeconomic stabilization are not possible} without a decisive break-through in privatization. We will sell 6 companies with more than 1,000 employees by end-March, another 6 by end-June (structural benchmarks) and bring 10 such companies to the stage of negotiations with a selected bidder by mid- 2003 . We will refrain from imposing employment preservation conditions in the privatization contracts, and will continue to attach a weight of at least 50 percent to the offered price in the bid evaluation process.

\section{We are aware that many large companies cannot be privatized unless their} overstaffing is reduced. We have therefore decided to reduce employment in 22 companies by over 18,000 positions (excluding any externalization) by end-June, 2003 (performance criterion). At least 6,000 employees will be made redundant by April 5, including 1,750 positions in Siderurgica (prior action). To alleviate social effects, we approved a special time-limited program that will provide severance payments to employees as described in paragraph 12 . This scheme will also be available for up to 2,000 layoffs in other companies in APAPS' portfolio. We will also impose a hiring freeze in these companies until end-year, effective April 1, 2003.

25. We will initiate liquidation procedures for companies with particularly weak financial results. We will immediately start liquidation procedures for the three companies Polirom, Siderca, and Sidermet. For the companies Corapet, Rulmentul Slatina, Verachim, Oltplast, Chimcomplex, Tepro, and Carom, the liquidation procedure will be initiated by end-June, unless privatized (structural benchmark).

26. Although the sale of BCR proved unsuccessful in the current business environment, we remain firmly committed to its privatization once market conditions have improved. The completion of the sale of a blocking minority share to the EBRD and the IFC will be an end-July structural performance criterion. To achieve this objective, on April 8 we provided the two IFIs complete access to all requested data, BCR staff and BCR auditors (prior action). By April 10, we will modify the bank privatization law and approve the revised privatization strategy. To facilitate the timely preparation of the 2002 accounting statements, the Ministry of Finance issued all necessary norms and regulations on February 20, 2003. We will share the preliminary 2002 balance sheet and income statement with the two IFIs by March 31, 2003. 


\section{E. Monetary Policy and Banking Issnes}

\section{Monetary policy will continue to be conducted in a managed float}

framework. The NBR will guide the exchange rate along a path consistent with our inflation target and a modest real appreciation. The current policy of less frequent but sizable interventions, allowing for wider exchange rate fluctuations, will continue, and the NBR will consider exchange rate movements of the leu against both the euro and the U.S. dollar. The targeted depreciation rate and further interest rate adjustments will be reconsidered after the effects of the minimum wage increase can be assessed with greater precision. Our 2003 monetary program also targets an increase in official reserves by US $\$ 1$ billion, to about $4 \frac{1}{4}$ months of prospective imports of goods and services. Velocity is expected to decline further, in line with continued progress in reducing inflation and strengthened confidence.

\section{The rapid growth in foreign-currency-denominated credit poses increasing}

financial risks. The effect of the December reserve requirement differentiation for lei and foreign currency to 18 percent and 25 percent, respectively (from the common level of 22 percent) has yet to fully materialize. The NBR will continue to exercise close supervision of the most active lenders, to ensure their adequate assessment of credit risk. The tightening of loan provisioning in January 2003 should also help moderate credit growth. If necessary, later in the year we will consider increasing capital requirements for lending in foreign currencies.

29. We have started to implement the measures recommended in the Safeguards Assessment report. We have clarified the reconciliation procedure between accounting statements and monetary data and submitted to the Fund the first and second such quarterly reconciliation, certified by the Internal Audit Department. An investment committee has been working in the NBR since September 2001 to exercise its foreign reserve management function. We are also actively implementing the other recommendations of the Safeguards Assessment report.

\section{F. Governance Issues}

30. Our efforts to improve the business climate continue. The parliament has approved in December 2002 an anti-corruption program and legislative changes required for its implementation are under way. We will conduct all privatizations in an open and transparent manner. In protecting the rights of minority shareholders, we will refrain from introducing measures that impose an undue burden on strategic investors. We will prepare a report on our efforts to prosecute individuals involved in financial wrongdoings, including FNI, Banca Romana de Scont, and other recent cases and share it with Fund staff, In cooperation with the World Bank, we will reassess the impact of the new Labor Code on labor market flexibility and amend it as needed. 
Table 1. Romania: Quantitative Perforrance Criteria and Indicative Targets for 2001-03 1/

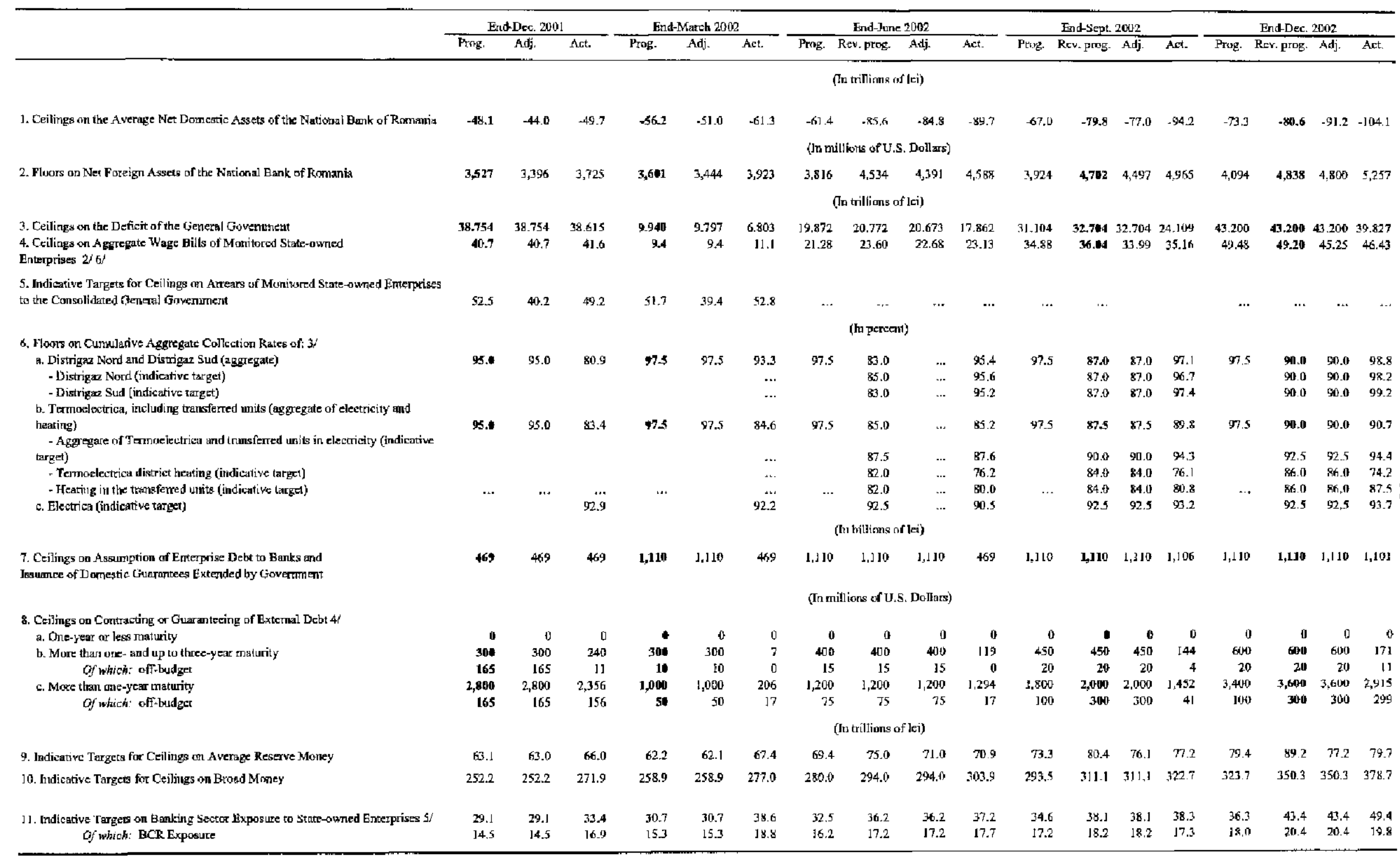

1/ The perfornanse criteria and indicative targets envisaged under the progran are defined in the Technical Memorandum of Urderstanding (TMU).

The figures in bold for end-Decenber 2001, end-March 2002, end-September 2002 (revised program), end-December 2002 (revised program) and end-June 2003 are performance criteria, unless indicated alterwise, while cond-June 2002 and cad-March 2003 figures are indicative tartots.

2 Adjusted to reflest the decreasc in the pool of monitored canpatites, owing to privatization and tretgers, from 86 of 82 in 2002 and to 76 in 2003.

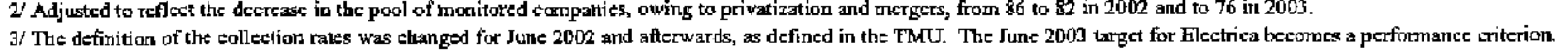

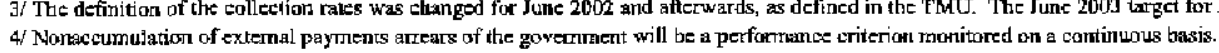

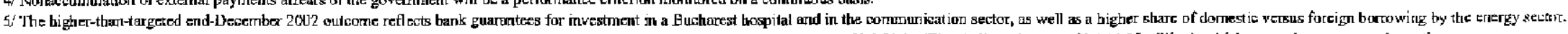

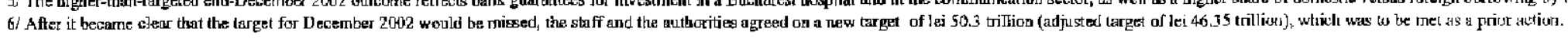

CInternational Monetary Fund. Not for Redistribution 


\begin{tabular}{|c|c|c|c|c|}
\hline & End-Mareh 2003 & End-Junc 200:3 & End-Seppt. 2003 & End-Dea 2003 \\
\hline & Prog. & Prog. & & \\
\hline 1. Ccilings an the Average Net Domestic Assets of the Kational Rank of Rornatia & -85.7 & $-1106,8$ & -114.0 & -106.7 \\
\hline 2. Flours on Net Foreign Assets of the National Bank of Romania & 5,522 & 6,075 & 6,410 & 6,310 \\
\hline 3. Ceilings on the Deficit of the General Guvenunent & 10.600 & 27.352 & 31.309 & 49.520 \\
\hline $\begin{array}{l}\text { 4. Ceilings on Aggregate Wage Bills of Moritored Stare-a waed } \\
\text { Enterprises } 21\end{array}$ & 12.84 & 26.5 & 39.82 & 53.15 \\
\hline \multicolumn{5}{|l|}{ 5. Indicarive Iurgels for Ceilings on Arrears of Monjitared Stete-owned Enterprises } \\
\hline to the Consolidaled Gencral Govemnent & $\cdots$ & $\cdots$ & $\cdots$ & $\cdots$ \\
\hline \multicolumn{5}{|l|}{ 6. Floors on Cumpulative Aggregate Collection Rates of: $3 /$} \\
\hline a. Distrigaz Nord and Distrigaz Sud (aggregate) & 94.0 & 95.0 & 95.0 & 96.0 \\
\hline - Distrigaz Nord (indicative target) & 94.0 & 95.0 & 95.0 & 96.0 \\
\hline - Distrigaz Sud (indiearive target) & 94.0 & 95.0 & 95.0 & 96.0 \\
\hline b. Tectroclectrica, including transferred imits [aggregate of electricity and & & & & \\
\hline 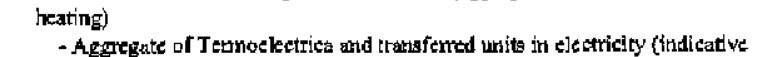 & 91.0 & 9I.0 & 93.5 & 95.0 \\
\hline targel) & 94.0 & 94.0 & 96.0 & 97.0 \\
\hline - Ternoelectrica district heating (indicatiwe target) & 84.0 & 84.0 & 89.0 & 92.6 \\
\hline - Heating in the transferred units (indicative targer) & 84.0 & 84.0 & B9.0 & 92.0 \\
\hline c. Elecrica & 94.0 & 95.0 & 96.0 & 97.0 \\
\hline $\begin{array}{l}\text { T. Ceilings on Assumption of Enterprise Debt to Banks and } \\
\text { lssuamee of Dowestic Guartanieces Extended by Govanument }\end{array}$ & 1,410 & 1,510 & 1,610 & 1,710 \\
\hline \multicolumn{5}{|l|}{ 8. Ceilings un Cuntracting or Gutatartecing of Extemel Debt 4 / } \\
\hline a. One-year or less maluily & 0 & ") & 0 & 0 \\
\hline b. More than one-and up to three-year maturity & 300 & 4010 & 450 & 600 \\
\hline of whith: oft-budget & 10 & 15 & 20 & 20 \\
\hline c. More than ore-year maturity & 805 & 2,1094 & 2,645 & 3,400 \\
\hline Of which: off-budget & 360 & stol & 600 & 650 \\
\hline 9. Indicative Targets for Ceilings on Average Resave Money & 96.2 & 103.4 & 15.0 & 124.9 \\
\hline 10, Indicative Targets for Ccilings on Broad Money & 385.7 & 415.5 & 448.0 & 506.2 \\
\hline 11. frudicative Targets on Banking Sector Exposure to State-owned Enterprises & 49.7 & 51.5 & 53.2 & 59.9 \\
\hline Of which: BCK Exposure & 22.4 & 23.3 & 23.9 & 27.3 \\
\hline
\end{tabular}

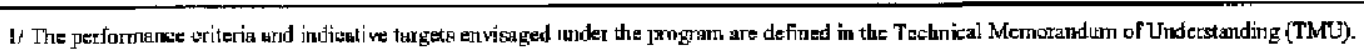

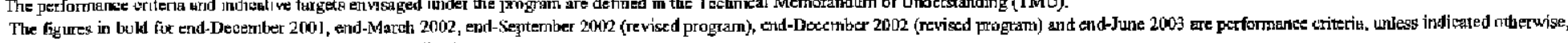
while end-June 2062 and end-March 2003 figunes are indicative targets.

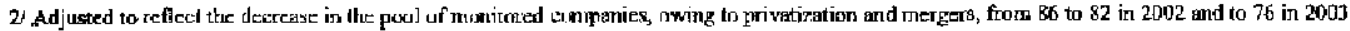

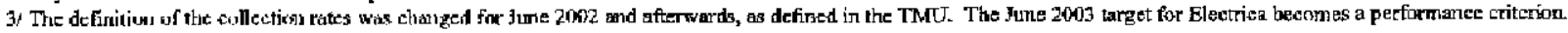

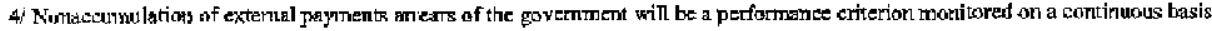




\section{Prior Actions:}

- Establishment of a tax administration department within the Ministry of Public Finance and appointment of the responsible Secretary of State, as described in paragraph 13.

- Approval of an Emergency Ordinance by November 20, 2002, specifying the intermediate steps for establishing a common agency for collection, audit, and enforcement of social sccurity contributions under the Ministry of Finance on January 1, 2004, as described in paragraph 13.

- Approval of an Emergency Ordinance canceling Articles 1-7, and Articles 15, 16 (b), 18, and 20 of Law 130/1999, thereby eliminating the legal basis for "civil contracts", as specified in paragraph 10 .

- Actual compliance with the revised target for the annual 2002 wage bill in the 82 monitored state-owned companies as discussed in paragraph 5.

- Signing of the contract with the privatization advisor for Petrom by November 1,2002 , as specified in paragraph 21.

- Announcement by November 10,2002 , of privatization tenders to acquire a majority share of at least 51 percent of two electricity distribution companies, in line with paragraph 21 .

- Privatization of four companies with more than 1,000 employees in Q4 of 2002.

- Approval of the restructuring plan of the parent company of Siderurgica by February 20 , 2003.

- Request by April 15, 2003 technical assistance from the World Bank and the Fund on the issues of medium-term adjustment in gas prices and the concomitant reform in the tax regime in the gas sector.

- Increase the unified end-user price for natural gas to the equivalent of US\$90/tcm as of March 1, 2003, as specified in paragraph 17.

- Pcrmission of the Privatization Commission by April 3, 2003 for a start of due diligence of BCR by EBRD/IFC, providing complete access to all required data, BCR staff and BCR auditors, in line with paragraph 26.

- Amendment by March 15, 2003 of Emergency Ordinance 208/2002 on the cancellation of arrears and exemption from tax and utility bill payments in line with paragraph 19 .

- Disconnection by March 25, 2003, of the largest industrial nonpayers to the three utilities Distrigaz Nord, Distrigaz Sud, and Electrica as described in paragraph 18 and Table 4.

- Implementation of 6,000 layoffs in 22 conpanies by April 5, 2003, including 1,750 layoffs in Siderurgica.

\section{Structural Performance Criteria:}

- Approval in December 2002 of the annual 2003 budgets of the monitored state-owned companies limiting the growth in their aggregate gross wage bill to 14 percent in nominal
Done.

Done.

Revised target exceeded by a small amount.

Done with delay on December 13.

Done with delay on January 8.

Done,

Done with delay on March 31.

Done.

Done on April 8 instead of April 3.

Done.

Done with delay on April 2.

Observed. 
Table 2 (continued). Prior Actions, Structural Performance Criteria and Benchmarks

terms over 2002 adjusted for any outsourcing.

- Adjust end-user electricity prices on October 1, 2002, January 1, 2003, April 1, 2003 and July 1, 2003 to keep them constant in US\$ terms at the level of July 1, 2002, as discussed in paragraph 17.

- Parliamentary approval by end-May 2003 of legislation amending the laws on the pension, unemployment, and health insurance funds (in particular law 19/2000, law 76/2002, and Emergency Ordinance 150/2002) to harmonize procedures for collection, enforcement and auditing.

- Implementation of 18,000 layoffs (cumulative) in the group of 22 companies by June 30 , 2003 , in line with paragraph 24 and Section IV of the TMU.

- Adjust Termoelectrica's producer price for clectricity to the equivalent of a minimum of US $\$ 39 / \mathrm{M}$ wh as of October 1,2002 and July 1,2003, as provided for in paragraph 17 .

- Increase the unified end-user price for natural gas to the equivalent of USS93/tem as of July 1,2003 , in line with paragraph 17.

- Adjust the National Reference Price for heating to the equivalent of US $\$ 20 / \mathrm{Gcal}$ as of July 1,2003 , as discussed in paragraph 17.

- Completion of the privatization of BCR by end-February 2003.

- Sign the contract for the sale of 25 percent plus two shares of the BCR capital to the EBRD and the IFC by July 31,2003 , in line with paragraph 26 .

\section{Structural Benchmarks:}

- The government will refrain from introducing tax holidays or any other new distortionary tax incentives or postponing the discontinuation of expiring ones.

- All transferred heating units should not receive fuel at Termoelectrica's expense.

- The largest industrial nonpayers to the three utilities will stay disconnected until full payments for all bills from December 2002 onwards have been received by the utilities.

- In addition to the sale of four companies with more than 1,000 employees envisaged as a prior action, four more such companies will be privatized by end-December 2002, another set of six by March 31, 2003 and six more by June 30,2003.

- Initiations of liquidation procedures for seven perennial loss-makers (Verachim, Oltplast, Corapet, Rulmentul Slatina, Chimcomplex, Tepro and Carom) on July 1, 2003, unless they are privatized by June 30 .

- Launching of the tenders for expressions of interest in the privatization of Petrom, in consultation with the World Bank, by June 30, 2003.
Observed on October 1 , 2002, January 1, 2003 and April 1, 2003.

Observed on October 1 , 2002.

Not met. Waiver requested

Continuous. The recent decision to cancel tax arrears of selected comparies implies nonobservance.

Continuous.

Continuous.

Partially met in Q4'2002 and Q1'2003. 
Table 3. The Authorities' Plan to Adjust Prices of Electricity, Heating and Natural Gas

\begin{tabular}{|c|c|c|}
\hline Energy Price & Adjustment & \\
\hline \multirow[t]{2}{*}{ End-aser Electricity Price } & Increase: & \\
\hline & (iin percent) & \\
\hline Oetober $1,200 \mathrm{~J}$ & 3.6 & $1 / 3 j$ \\
\hline Noycmber 1, 2001 & 3.6 & at $3 /$ \\
\hline December 1,2001 & 3.6 & $2 ! 3 !$ \\
\hline January I, 2002 & 3.6 & $2 / 31$ \\
\hline February 1,2002 & 3.6 & $2 / 3 /$ \\
\hline March 1, 2002 & 3.6 & $2 f \quad 4 /$ \\
\hline Apri] 1, 2002 & 14.0 & If 3 ! \\
\hline July 1,2002 & 1.5 & $1 / 3 /$ \\
\hline
\end{tabular}

End-user elcetricity prices will be adjusted on October 1, 2002, Járuary 1, 2003, April 1, 2003 and July 1, 2003 to keep them constant in U.S. dollar terms at the level as of July 1, 2002.

Oetober 1, 2002

January !, 2003

Apti\} I, 2003

July 1,2003

Electriejty Producer Price for Termoelectricn

April 1, 2002

July 1,2002

October 1, 2002

Jamary 1, 2003

April 1,2003

July 1,2003

Heat Producer Price for Termoelectrica

January 1,2002

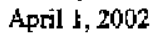

July 1,2002

July 1,2003

National Reference Price for Heating

January 1, 2002

April 1,2002

July 1, 2002

July 1, 2003

\section{Unified End-user Nutural Gas Price}

October 1, 2001

Junuary 1, 2002

April 1, 2002

July 1,2002

October ]. 2002

March 1, 2003
Oclober 1, 2001

July 1,2003

Adjust price to the cquivalent of U.S. dollars

$\begin{array}{lll}39.00 & 1 / & 3 / \\ 39.00 & 1 / & 3 / \\ 39.00 & 2 t & 3 / \\ 39.00 & 1 / & 3 / \\ 39.00 & 1 / & 3 / \\ 39.00 & 6 / & \end{array}$

Adjust price to the equivalent of U.S. doilars

\begin{tabular}{|c|c|}
\hline 15.00 & $1 / 3 /$ \\
\hline 15.00 & $2 f$ \\
\hline 15.00 & $1 i$ \\
\hline 20.00 & 11 \\
\hline 20.00 & $\mathrm{l} /$ \\
\hline
\end{tabular}

$\Lambda$ djust price to the equivalent of U.S, dollars

$\begin{array}{lll}15.40 & 2 / & 3 \prime \\ 15.40 & 1 / & 3 / \\ 20.00 & 1 / & 3 \prime \\ 20.00 & 6 / & \end{array}$

Adjust price to the equivalent of U.S. dollars

$\begin{array}{lll}82.50 & 1 / & 3 / \\ 82.50 & 1 / & 3 / \\ 82.50 & 1 / & 3 / \\ 82.50 & 1 / & 3 / \\ 82.50 & 2 / & 3 / \\ 90.00 & 5 / & 3 / \\ 93.00 & 6 / & \end{array}$

1/ Indicative target.

2/ Structural performance criterion.

3) Implemented.

4/ Not observed.

5/ Prior action.

6/ New structural performance criterion. 
Tablc 4. Billings, Payments and Disconnection Status of Selected Customers of Natural Gas and Electricity Utilities

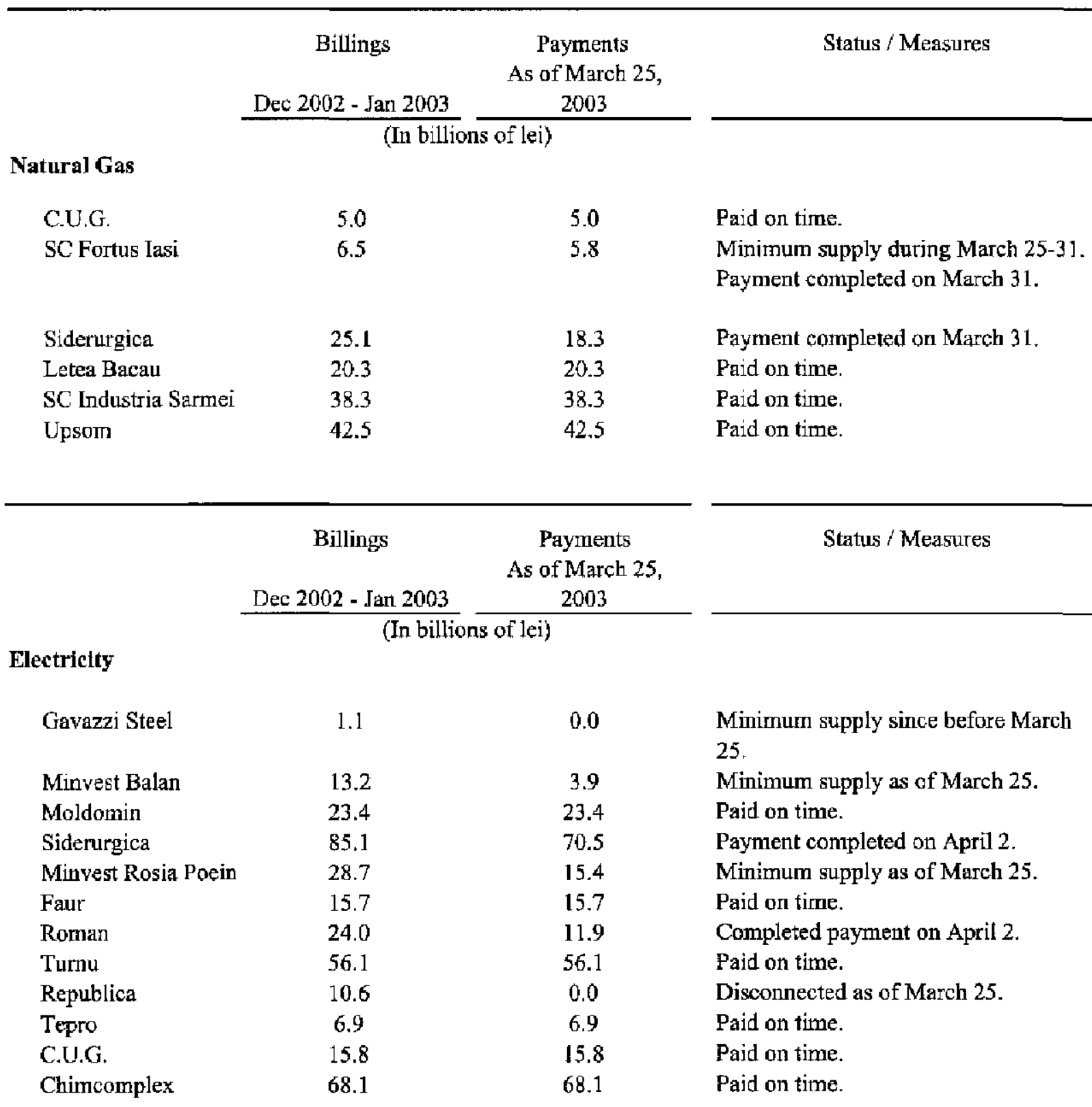




\section{SUPPLEMENTARY TECHNiCal MEMORANDUM OF Understanding FOR STAND-By ARRANGEMENT}

I. Ceilings on the Average Net Domestic Assets of the National Bank of Romania

II. Targets for Floor on Net Foreign Assets of the National Bank of Romania

III. Ceilings on the Cumulative Deficit of the Consolidated General Government

IV. Ceilings on Aggregate Wage Bill of Monitored State-Owned Enterprises and List of Twenty-two State-Owned Companies to Operate Layoffs

V. Indicative Target for Ceilings on Arrears of Monitored State-Owned Enterprises to the Consolidated General Government

VI. Floors on Cumulative Aggregate Collection Rates of Distrigaz Sud, Distrigaz Nord, and Termoelectrica

VII. Ceilings on the Assumption of Enterprise Debt to Banks by the Consolidated General Government and on the Issuance of Domestic Government Guarantees on Bank Lending to Enterprises

VIII. Ceilings on Contracting or Guaranteeing of External Debt

LX. Indicative Targets for Ceilings on Average Reserve Money

X. Indicative Targets for Ceilings on Broad Money

XI. Indicative Targets for Ceilings on Banking Sector's Total Exposure to State-Owned Enterprises 


\section{Ceilings on the Average Net Domestic Assets of the National Bank of Romania}

The average net domestic assets of the National Bank of Romania (NBR) are defined as the difference between average reserve money (as defined in Section IX of this attachment) and average of net foreign assets (as defined in Section II of this attachment for the indicated month, excluding the adjustment for foreign currency denominated treasury bills), both expressed in local currency. Average net foreign asset stocks will be converted into lei for the purposes of calculating average net domestic assets at the average monthly lei/U.S. dollar rates specified in consultation with Fund staff. The average stock of NFA is defined as the average of the daily NFA as defined in Section II. The limits will be monitored from daily data on the accounts of the NBR supplied weekly to the IMF by the NBR. The average NDA as of September 2001 was lei 39,559 billion.

The ceiling on average net domestic assets of the NBR will be adjusted under the following circumstances:

(1) Downwards (upwards) in a proportional fashion, for the fraction of the month that gross foreign financing exceeds (falls short of) programmed levels, specified in Section II.

(2) For any change in reserve requirements as described in Section IX. Before undertaking any such changes, the NBR will consult IMF staff.

(3) Upwards (downwards) in a proportional fashion, by the lei equivalent of the decrease (increase) in the stock of foreign currency denominated Treasury bills (cumulative from end-December 2002).

(4) Downwards in a proportional fashion by the lei equivalent of the increase in foreign currency receipts from large privatizations (sale price above $\$ 10$ million) (cumulative from end-December 2002)

(5) Downwards by the shortfall in actual reserves from required reserves for any individual bank.

\section{Targets for Floor on Net Foreign Assets of the National Bank of Romania}

Net foreign assets of the NBR consist of reserve assets minus foreign liabilities. For the purposes of the program, reserve assets shall be defined as monetary gold, holdings of SDRs, any reserve position in the IMF, and holdings of foreign exchange in convertible currencies by the NBR. Excluded from gross reserves are long-term assets, NBR redeposits at the commercial banks, any assets in nonconvertible currencies, encumbered reserve assets, reserve assets pledged as collateral for foreign loans, reserve assets pledged through forward contracts, and precious metals other than gold. Monetary gold shall be valued at an accounting price of US\$280.4 per ounce and SDRs at US\$1.355109 per SDR. NFA stocks are measured at the last working day of the respective month. 
For the purposes of the program, foreign liabilities shall be defined as loan, deposit, swap (including any portion of the NBR gold that is collateralized), and forward liabilities of the NBR in convertible currencies including, foreign currency deposits of resident commercial banks at the NBR; IMF purchases; borrowing from international capital markets; and bridge loans from the BIS, foreign banks, foreign governments, or other financial institutions, irrespective of their maturity.

All assets and liabilities denominated in convertible currencies, other than the U.S. dollar, shall be converted at their respective exchange rates against the U.S. dollar on December 31 , 1999. All changes of definition or valuation of assets or liabilities as well as details of operations concerning sales, purchases, or swap operations of gold shall also be communicated to the IMF staff.

The NFA of the NBR will be adjusted:

(i) upwards/downwards by 100 percent of the excess/shortfall of gross foreign financing ${ }^{1}$ from the programmed levels:

on a cumulative basis from end-September 2001:

$\begin{array}{ll}\text { December } 2001 & \text { US\$248 million } \\ \text { March } 2002 & \text { US\$248 million } \\ \text { June } 2002 & \text { US\$953 million } \\ \text { September } 2002 & \text { US\$953 million } \\ \text { December } 2002 & \text { US\$953 million }\end{array}$

on a cumulative basis from end-December 2002:
March 2003
US\$50 million
June 2003
US\$450 million

(ii) by the change in the stock of foreign currency denominated Ministry of Finance Treasury Bills including those issued for bank restructuring (on a cumulative basis from endDecember 2002). The outstanding stock on December 31, 2002 was $\$ 478.9$ million evaluated at the program exchange rates.

(iii) upwards by the amount of foreign currency receipts from large privatizations (sale price above $\$ 10$ million) (cumulative from end-December 2002).

The NFA will be monitored on the basis of daily operational data through to end-March 2002, after which monetary survey data will be used. Daily data will still be used to calculate average NFA. All data is provided by the NBR. The NFA figure was US\$ 3,311 million on September 30, 2001, and US\$ 5,257 million on December 31, 2002.

\footnotetext{
${ }^{\mathrm{I}}$ Foreign financing is defined as disbursements of balance of payments support loans to the government with a maturity of more than a year from multilateral and bilateral creditors and resources with a maturity of more than one year raised in the international capital markets by the government. This excludes use of IMF resources.
} 


\section{Ceilings on the Cumulative Deficit of the Consolidated General Government}

The consolidated general government includes the state budget; the budgets of the local authorities; the social protection funds; ${ }^{2}$ the "Special Fund for Modernizing Roads", the "Special Fund for the Development of the Energetic System", the "Special Reinsurance Fund", the "Authority for Privatization" (APAPS), the "Fund for the Development of Romanian Agriculture", the "National Administration of Roads (NAR)", other extrabudgetary funds managed by the Ministry of Finance or other Ministries and agencies outside the budgetary framework; other extra-budgetary operations of ministries financed by foreign loans; and the counterpart funds created from the proceeds of foreign loans. Any new funds created during the program period to undertake operations of a fiscal nature as defined in the IMF's Manual on Government Finance Statistics will be incorporated within the definition of consolidated general government.

Under the program, the deficit of the consolidated general government will be measured based on revenue and expenditure data provided by the Ministry of Finance, and also based on "below the line" data, that is, by the sum of domestic and external financing of the budget as well as privatization proceeds received by all entities of the consolidated general government and proceeds from the recovery of bank asset by AVAB. All efforts will be made to reconcile the measurement of the deficit from "below" and from "above the line". However, should these efforts not succeed in eliminating the discrepancies, the respectively higher deficit number will be used for program purposes.

For program purposes, net credit of the banking system to the consolidated general government is defined as all claims of the banking system on the consolidated general government less all deposits of the consolidated general government with the banking system. Foreign-currency denominated credit to government outstanding at

December 31, 2001 will be converted in U.S. dollars at the end-December 1999 exchange rate and from dollars into lei using the rates specified in consultation with Fund staff. Foreign-currency denominated credit newly issued in 2001 and 2002 will be valued at the exchange rates specified in consultation with Fund staff. Government loans to banks at an interest rate less than the reference rate of the NBR to finance on lending to economic agents are excluded from government deposits; an agreed listing of the accounts to be treated as government deposits for program purposes is contained in the FAD aide memoire "Romania: Measuring the Fiscal Deficit", Part II, Appendix 11, February 1994.

For program purposes, the ceilings on the quarterly cumulative deficit of the consolidated general government in 2003 will be adjusted downwards by a shortfall of the cumulative quarterly privatization proceeds received by the consolidated general government. Privatization receipts denominated in foreign currency will be converted into U.S. dollars using the exchange rates on December 31, 1999, and from dollars into lei using the rates specified in consultation with Fund staff. The floor for these proceeds is as follows:

\footnotetext{
${ }^{2}$ These include the State Social Security Fund, the Insurance Fund for Work-Related Accidents, the Unemployment Fund, and the Health Social Insurance Fund.
} 
(In billions of lei)

Cumulative quarterly privatization proceeds of the general consolidated government for 2003:

March 31, 2003 (minimum target)

June 30, 2003 (minimum target)

September 30, 2003 (minimum target)

December 31, 2003 (minimum target)
1,000

2,000

3,000

4,000

To achieve the deficit target of lei 49,520 billion in 2003 and to ensure sufficient room for a downward adjustment of this target in the course of 2003 , should this prove necessary to contain the effects of the minimum wage increase, the cumulative monthly expenditure entitlements for the state budget during January-June 2003 will be limited to the ceilings agreed with Fund staff. In addition, should the cumulative monthly revenues of the general consolidated budget fall short of the revenue targets agreed with Fund staff, the monthly state budget expenditure ceilings will be adjusted downwards to compensate for this shortfall.

Consolidated General Government

(billion of lei)

(excluding "National Administration of Roads";

cumulative total revenues including grants)

January 31, 2003 (actual)

44,246

February 28, 2003 (indicative revenue target)

85,732

82,348

March 31, 2003 (indicative revenue target)

128,797

April 30, 2003 (indicative revenue target)

177,248

May 31, 2003 (indicative revenue target)

221,602

June 30, 2003 (indicative revenue target)

267,507 
Agreed expenditure

Actual

Ceilings

State Budget

(billion of lei)

(cumulative total expenditure)

January 31, 2003 (actual)

19,616

February 28, 2003 (indicative ceiling)

39,901

38,042

March 31, 2003 (indicative ceiling)

65,215

April 30, 2003 (indicative ceiling)

88,987

May 31, 2003 (indicative ceiling)

113,120

June 30, 2003 (indicative ceiling)

137,764

\section{Ceiling on Aggregate Wage Bill of Monitored State-Owned Enterprises and List of Twenty-Two State-Owned Companies to Operate Layoffs}

\section{A. Ceiling on Aggregate Wage Bill of Monitored State-Owned Enterprises.}

The set of 76 state-owned enterprises, whose wages are to be monitored under Emergency Ordinance 79/2001, is specified in Government Decision 866/2001. The wage bill of this set of state-owned enterprises has been adjusted for the decrease in the number of enterprises from 82 in 2002 and 86 in 2001.

The wage bill will be adjusted as follows:

(i) downwards by the amount of savings due to "externalization" (defined as the spinning off of a unit or its transfer to another entity, or temporary/permanent transfers of employees when the sum of these transfers exceeds 100 employees per month). In each month, savings from externalization will be calculated on a company by company basis as the product of the number of externalized employees so far and the company's average wage.

(ii) if a company is privatized, downwards by the budgeted wage bill of the privatized company, starting with the month following the month of signing the privatization contract.

The wage bills will be measured on a cumulative basis across the different sectors, on a monthly basis. The Ministry of Labor and Social Protection will undertake the responsibility of collecting data from the various line ministries (RAs and national companies) and APAPS (commercial companies), and will report the wage bills and employment figures for each of the monitored enterprises (including aggregate figures for each ministry and for the overall total) to the IMF on a monthly basis. Employment reduction resulting from all forms of 
outsourcing will be reported in the "externalization" column of the respective tables, with a footnote, if necessary.

\section{B. List of Twenty-Two State-Owned Companies to Operate Layoffs}

The set of 22 state-owned companies that will operate at least 18,000 layoffs, as discussed in paragraph 24 of the SMEFP-2, consists of:

1. Tractonul-UTB SA Brasov.

2. Turnu SA Turnu Magurele.

3. Electroputere SA Craiova.

4. CSR SA Resita.

5. Republica SA Bucharest.

6. Iasitex SA Iasi.

7. Mefin SA Sinaia.

8. Mecanica SA Marsa.

9. Chimcomplex SA Borzesti.

10. Letea SA Bacau.

11. Rempes SA Deva.

12. CUG SA Cluj.

13. Fortpres SA Cluj Napoca.

14. Siderurgica SA Hunedoara.

15. Tepro SA Iasi.

16. Fortus SA Iasi.

17. ARO SA Campulung.

18. Carom SA Onesti.

19. Roman SA Brasov.

20. Faur SA Bucharest.

21. Industria Sarmei SA Campia Turzii

22. Rulmentul SA Brasov

\section{Indicative Target for Ceilings on Arrears of Monitored State-owned Enterprises to the Consolidated General Government}

The ceiling applies to the outstanding stock of arrears of the set of 76 monitored state-owned enterprises, whose arrears are to be monitored under Emergency Ordinance 79/2001 and Government Decision 866/2001. Under the ordinance, arrears are defined as accounts payable past the due date stipulated explicitly in the contracts, or if no such explicit date exist, 30 days after services/products are provided. The reporting on total arrears will have the following subcategories: to the state budget, to the social security budget; to the local budget; to special funds; and to other creditors. Arrears to the consolidated general government are defined as the sum of the first four categories. Amounts reflecting tax arrears exclusive of penalties will be reported separately. For arrears which have been rescheduled/canceled, the rescheduled/canceled amounts (including penalties) will not be counted as arrears reduction, and has to be reported. The report will include a breakdown of arrears to the ten largest creditors for each company. The report will also include data on overdue claims of each of the monitored companies, as reported under Emergency Ordinance 
$79 / 2001$. For changes to the set of monitored companies, the targets will be adjusted downwards/upwards by the amount of arrears of the companies removed/added to the set. Data for monitoring purposes shall be supplied monthly to the IMF by the Ministry of Finance. The stock of arrears at end-December 2000 was lei 47.2 trillion.

\section{Floors on Cumulative Aggregate Collection Rates of Distrigaz Sud, Distrigaz Nord, Termoelectrica and Electrica}

Floors will be set on the cumulative collection rates of the following companies:

- the combined rate (performance criterion) of Distrigaz Nord and Distrigaz Sud (indicative targets on the individual company collection rates);

- on the combined collection rate for heating and electricity of Termoelectrica, including the production units transferred to local authorities (performance criterion). Indicative targets will be set on the collection rates for (i) electricity for both Termoelectrica and the production units transferred to local authorities; (ii) Termoelectrica's district heating; (iii) heating in the transferred units; - on Electrica's collection rate (indicative target till March 2003, performance criterion as of June 2003).

The floors and outcomes for end-September, end-December 2001, and end-March 2002 are measured using cumulative collection rates defined as the ratio of collections to billings, measured from the start of the year to the specified date. For the remaining test dates floors on collection rates are defined as follows:

(i) Termoelectrica and local anthority units (Heating sector), Distrigaz Nord and Sud: Heating and gas bills are lagged by one month. Definition of 12-month moving collection rate $\mathrm{c}(\mathrm{m})$ for the month $\mathrm{m}=1,2 . .12$.

$$
\begin{array}{ll}
c(m)= & \text { sum(heating collections }(m) \text { :heating collections }(m-12) \text { divided by } \\
\text { sum(heating bills }(m-1): \text { heating bills }(m-13)) .
\end{array}
$$

(ii) Termoelectrica and local authority units (Electricity sector); Electrica; Definition of 12-month moving collection rate $\mathrm{c}(\mathrm{m})$ for the month $\mathrm{m}=1,2 . .12$ :

$$
\begin{aligned}
& c(m)= \text { sum(electricity collections }(m) \text { :electricity collections(m-12) divided by } \\
&\text { sum(electricity bills }(m) \text { :electricity bills(m-12) }) .
\end{aligned}
$$

Using these definitions the collection rate of Termoelectrica at end-December 2001 was 85.5 , of Electrica 92.9, and of the two gas companies, 85.5.

Data for these companies will be collected by the Ministry of Industry and reported to the IMF on a monthly basis. The Ministry of Industry will include in this report data on billings and collections registered by Distrigaz Nord, Distrigaz Sud, Electrica and Termoelectrica, as well as information on possible dis- and reconnections for the following industrial (a) and heating (b) companies. 
a) SC Siderurgica, COS Targoviste, Minvest SM-Rosia Poieni, Moldomin, Minvest-SM, Balan, Snif, SC Industria Sarmei, Gavazzi Stee], Minvest-SM Baia de Aries, SC Turnu, CUG Cluj, SC Apaterm Galati, SC Tractorul UTB, SC Chimcomplex, Minvest- SM Brad, Apa Nova (RGAB), Minvest -SM Coranda Certej, Minvest -SM Poiana Rusca Teliuc, Siderea, SC Electrocarbon, Tepro, Nitramonia, Viromet, Amonil, Oltchim, Sere Codlea, US Govora, Republica, Zahar Bod, Stirom Bucuresti, Danubiana, Gerom Buzau, Colorom Codlea, Roman Brasov, Metrom Brasov, Carfil Brasov, Stiaz Azuga, Faur Bucuresti, UPSOM SA Ocna Mureș, Bicapa SA Târnăveni, SC Ind.Sârmei C.Turzii, SC Stipo SA Dorohoi, Ampellum SA Zlatna, SC Cugir SA, SC Melana Săvineşti, Letea Bacau, Rafo SA Oneşti, SC Fortus SA Iaşi, Ambro SA Suceava, Stratusmob SA Blaj, SC Sticla Turda, Jris SA Cluj, Metalurgica Aiud.

b) Radet Bucuresti,Radet Constanta, Apaterm Galati, RA Termoficare Craiova, SC Apaterm, SA Deva, Termica SA Targoviste, Termoficare Petrosani, Dalkia Ploiesti, SC Termoficare Petrosani, SC Universal Lupeni, Aptercol Braila, SC Citadin Aninoasa, RA Termoficare Cluj, SC Aqua Calor P. Neamt, RA Energomur Tg Mures, SC Energ. Temica Sibiu, Temoloc Populatie Bacau, RA Goscom Roman, Proditerm Bistrita, Rail Hunedoara, Comunala RA Satu Mare, Termica SA Botosani, Radet Bucuresti, Enet Focsani, Cet Braila, Cet Govora, RA Termo Craiova, Ram Buzau, RA Termo Brasov, Aquaterm Tg. Jiu, Aquaterm 98 Pitesti.

\section{Ceilings on the Assumption of Enterprise Debt to Banks by the Consolidated General Government and on the Issuance of Domestic Government Guarantees on Bank Lending to Enterprises}

The ceilings apply to the cunulative stock from end-September 2001 of newly guaranteed or assumed domestic debt by the consolidated general government. For program purposes, the assumption of enterprise debt to banks by the consolidated general government and the issuing of a guarantee to assume enterprise debt to banks are treated as being equivalent. This limit includes any loan on which the government pays or guarantees interest, even if the principal is not guaranteed. The consolidated general government is defined in Section III of this attachment. The criterion also applies to the use of APAPS resources for recapitalizing enterprises or as collateral for bank loans. Foreign currency denominated loans will be converted at accounting exchange rates specified in consultation with Fund staff. These limits exclude:

- the contracting or guaranteeing of external debt, for which separate limits are set out in Section VIII of this attachment;

- debt transferred in the process of bank restructuring, privatization or liquidation of stateowned enterprises.

- the assumption of debt as a result of an activation of a guarantee or collateral.

- domestic guarantees for loans for fuel imports for the 16 heat-producing units which were 
transferred from Termoelectrica to local authorities, to the extent that external off-budget guarantees issued for this purpose (see Section VIII) fall short of US\$120 million (i.e., the sum of domestic and external guarantees issued for this purpose must not exceed US\$ $\$ 20$ million).

Data for monitoring purposes shall be supplied monthly to the IMF by the Ministry of Finance. The stock of guarantees and debt assumed as described in this section was lei 469 billion as of end-September 2001 .

\section{Ceilings on Contracting or Guaranteeing of External Debt}

The ceilings apply to the cumulative stock for each year of newly contracted or guaranteed external debt by the consolidated general government. The consolidated general government is defined in Section III of this attachment. This performance criterion applies not only to debt as defined in point No. 9 of the IMF Guidelines on Performance Criteria with Respeet to Foreign debt adopted on August 24, 2000 (Executive Board Decision No. 12274-(00/85)) but also to commitments contracted or guaranteed for which value has not been received. The ceilings also apply to any assumption of loans for debt outstanding which were not previously contracted or guaranteed by the consolidated general government. Excluded from the ceilings are liabilities to the IMF and bridge loans from the BIS, foreign banks, foreign governments, or any other financial institution. Debt falling within the ceilings shall be valued in U.S. dollars at the exchange rate prevailing at the time the contract or guarantee becomes effective. Loans considered concessional are also excluded from the ceilings. Offbudget debt includes all debt to non-budget entities from private sector creditors guaranteed by the Ministry of Finance. Loans for fuel imports for Distrigaz, Termoelectrica, and the 16 heat-producing units which were transferred from Termoelectrica to local authorities are included in the overall ceilings, while being excluded from the off-budget guaranteed debt ceilings. With respect to new loans contracted to ensure fuel imports for the heating period $2002 / 2003$, they are excluded from the off-budget guaranteed debt ceilings up to an amount of US\$200 million for Termoelectrica and US\$120 million for the transferred heating units. Fuel import loans contracted by Termoelectrica and the transferred beating units are excluded from the ceilings on debt with maturities between one and three years.

Concessional loans are defined as those with a grant element of at least 35 percent of the value of the loan, using currency-specific discount rates based on the commercial interest reference rates reported by the OECD (CIRRS) in effect at the time of contracting or guaranteeing the loan.

The ceilings shall be monitored from data supplied monthly to the IMF by the Ministry of Finance. The stock of debt at end-June 2001 was $\$ 1,194$ million for maturities over one year (\$83 million of which was off-budget), \$196 million for the subceiling of debt with maturity of one to three years (none of which was off-budget), zero for debt with less than one year maturity.

Nonaccumulation of external payments arrears of the government will be a performance criterion monitored on a continuous basis. For program purposes, arrears with respect to 
called-up sovereign loan guarantees are defined as external payments overdue more than 30 days.

\section{Indicative Targets for Ceilings on Average Reserve Money}

Average reserve money is defined as the sum of average currency in circulation outside the NBR and average deposits (required plus excess reserves) of the commercial banks at the NBR for the indicated month. Commercial bank deposits exclude required and excess reserves it foreign exchange for foreign exchange deposits. Data on reserve money will be monitored from the daily indicators data of the NBR, which shall be supplied to the IMF weekly by the NBR. The stock of average reserve money as of September 2001 was lei 60,442 billion.

The ceilings on average reserve money will be adjusted in the following circumstances:

(1) Should reserve requirements be increased/decreased from 25 percent on all required reserves beld in lei, the reserve money targets would be increased/decreased by the product of the change in the reserve requirements and the programmed deposits for which required reserves are held in lei. The level of the programmed deposits is lei 189,866 billion for March 2003 and lei 204,549 billion for June 2003.

(2) The reserve money targets will be lowered by the shortfall in actual reserves from required reserves for any individual bank, measured from the $24^{\text {th }}$ of the previous month to the $23^{\text {rd }}$ of the test-date month, as provided for in the relevant NBR regulation.

\section{Indicative Targets for Ceilings on Broad Money}

Broad money is defined as the liabilities of the banking system with the non-bank public. Broad money includes foreign currency deposits of residents, but excludes government deposits and deposits of foreign monetary institutions and other non-residents. For the purposes of the program, deposits which are denominated in foreign currency will be converted into lei at the accounting exchange rates specified in consultation with Fund staff.

Data on broad money will be monitored from the monthly data on the accounts of the banks and the banking system, which shall be supplied to the IMF monthly by the NBR. The stock of broad money at program exchange rates as of September 30, 2001 was lei 235,363 billion.

\section{Indicative Targets for Ceilings on Banking Sector's Total Exposure to State- Owned Enterprises}

Total exposure covers all loans, advances, holdings of debt and off-balance sheet exposure of resident banks to state-owned enterprises. Data on loans will also be reported separately from total exposure. State-owned enterprises are all regis autonomes and commercial companies with majority ownership by the government or APAPS. For the purposes of monitoring, foreign currency denominated debt will be converted in lei at end-month lei/dollar exchange rates specified in consultation with Fund staff. Foreign currency denominated credit in 
convertible currencies, other than the U.S. dollar, shall be converted at their respective exchange rates against the U.S. dollar as specified in Section II of this attachment. Data on banking sector lending to state-owned enterprises will be monitored from monthly data provided by the NBR.

The amount of total exposure, as reported by the NBR, will include (on a cumulative basis from end-March 2002):

(i) exposure to companies where the majority ownership shifted to the private sector. For this purpose, APAPS and the relevant ministries will provide a monthly update of their portfolio to the NBR;

(ii) any amount of debt or off-balance sheet write-offs;

(iii) any assumption of debt or off-balance sheet items by the government or other public bodies.

Additionally, the NBR will report monthly on total exposure of the banking system to stateowned enterprises with outstanding exposure over lei 100 billion, on a company-by-company basis. The stock of banking sector exposure to state-owned enterprises at program exchange rates as of September 30,2001 was lei 27,052 billion of which lei 13,541 billion was to BCR. 


\section{ROMANLA}

\section{Third Review Under the Stand-By Arrangement and Requests for Waiver of Performance Criteria, Extension of Arrangement, and Rephasing of Purchases Supplementary Information}

Prepared by the European I and the Policy Development and Review Departments Approved by Susan Schadler and Liam P. Ebrill

April 24, 2003

1. This supplement reports on new information that has become available since the issuance of the staff report (EBS/03/48,04/11/03). The staff appraisal remains appropriate and the staff reaffirms its recommendation that the review be completed. All prior actions were met well abead of the five day deadline before the Board meeting. However, end-March monetary indicative targets were not observed, suggesting a need for some tightening of liquidity.

2. Macroeconomic developments remain consistent with the program, despite some weakening of activity. Inflation in March remained subdued at 1.1 percent, fully consistent with the authorities' end-year target of 14 percent. In February, industrial production declined by 2 percent on a 12 -month basis, for the first time since April 2002, reflecting the effects of weak growth in the EU, but also disruptions in supply in the metallurgy sector. Net wages in monitored state-owned enterprises (SOEs) in February were higher by $11 \frac{1 / 4}{4}$ percent in real terms than a year ago. In addition to the effects of the minimum wage increase, this figure reflected the conclusion of many collective contracts, and the corresponding adjustment in wages, somewhat earlier in the year compared with 2002 . Staff expect wage growth to moderate as the year progresses.

3. The general government deficit in Q1 was substantially below the target, but the government has postponed the decision on increasing fuel excise tax rates. Accoring to preliminary data, following good performance in January and February, revenue was weaker than expected in March, and the collections for Q1 were below the program. As spending was contained, the deficit of the general government came out at 0.3 percent of annual GDP, about $1 / 4$ percent of GDP less than targeted. Unfortunately, the government also decided to postpone the decision on restoring fuel excises back to their pre-February levels, which was agreed in the Supplementary Memorandum on Economic and Financial Policies. Instead, they decided to implement several measures to address large scale smuggling of fuels, including by limiting imports to specific custom posts and prosecuting non-payment of excises under 
the criminal code. Should the collections not improve by early June, the authorities have announced that excises would be raised as of June 15 . While welcoming the measures against smuggling, staff regret the decision not to increase the excise tax rates, which was expected to provide about 0.15 percent of GDP in additional revenue, and note that this action would likely necessitate other compensatory measures to ensure that the 2003 budget deficit target is met.

4. The indicative monetary policy targets for end-March were not observed. NFA remained flat in Q1, and the floor was missed by about US\$170 million. NDA exceeded its ceiling by 8 percent, and reserve money was 3 percent above target. Facing larger than expected outflows, the nature of which has yet to be identified, the NBR gave priority to sticking to its main disinflation tool, the exchange rate, instead of purchasing foreign exchange in the market, taking comfort from the large overperformance in NFA over the last two years. As a result, the exchange rate path has remained broadly in line with the program. The authorities attributed the overshooting of the reserve money target to an increase in demand for currency as a reduction in the number of NBR cash distribution offices has made the access to cash in some areas more difficult.

\section{In staff's view, the NBR should refrain from further cuts in interest rates and} strengthen efforts to sterilize liquidity. In February, staff advised the authorities against reducing the policy interest rate until the effects of the minimum wage increase could be better assessed. However, the rate dropped from 20 to 19 percent on average in March, while the NBR did not accept all bids at its deposit-taking auctions, effectively further easing monetary conditions. During the recent Spring meetings, the authorities agreed that additional efforts needed to be made toward mopping up liquidity in the banking sector, including increasing intake at deposit auctions. Since mid-April, the policy interest has been maintained at 20 percent.

\section{The effect of recent further slowdown in Europe on Romania is expected to be} modest. The robust export performance in 2002 suggests that the moderate export growth projected in 2003 remains within reach, although the downside risks for export and GDP growth are now stronger. On the other hand, the lower-than-expected trade deficit in January-February and the recent downward revision in international oil price projections for 2003 provide a welcome cushion in the current account projections. 


\section{IMF Approves US\$76 Million Disbursement Under Stand-By Credit with Romania}

The Executive Board of the International Monetary Fund (IMF) today completed the third review of Romania's economic performance under the 18-month Stand-By Arrangement, and approved the extension of the arrangement until October 15,2003. The decision will enable Romania to draw SDR 55.1 million (about US $\$ 76$ million) from the IMF immediately.

The Stand-By Arrangement was approved on October 31, 200l (see Press Release No. 01/43) for a total amount of SDR 300 million (about US\$413 million). So far, Romania has drawn SDR 134.7 million (US\$186 million) under the arrangement from the IMF.

Following the Executive Board discussion, Amne Krueger, First Deputy Managing Director and Acting Chair, said:

"Romania's macroeconomic performance was favorable in 2002. Economic growth was strong, inflation continued to decline, and the external current account balance improved significantly. These achievements stemmed from sound macroeconomic policies and progress in addressing structural weaknesses, notwithstanding slippages in enforcing financial discipline in state-owned enterprises and delays in privatization. The government is committed to implementing measures to consolidate macroeconomic stabilization, ensure robust and lasting economic growth, and prepare for EU accession. Key elements of the reform effort include pursuit of a restrained public sector wage policy, further reduction in state-owned enterprise losses, and more decisive progress in privatization.

"The uncertainties surrounding macroeconomic developments increasec' somewhat in early 2003 , owing to the large increase in the minimum wage in January and a more unfavorable external environment. Against this background, full and firm implementation of cautious wage policies will be vital to preserve macroeconomic stability and avoid a significant deterioration in Romania's external competitiveness in the medium term. Should unexpected pressures on prices or the current account develop because of the minimum wage hike, macroeconomic policies will need to be tightened.

"The 2003 budget continues to emphasize fiscal consolidation, while allowing for a substantial cut in payroll taxes, increased capital spending, and a doubling of heating subsidies to support low-income households. Looking forward, revenue collection needs to be further strengthened 
by implementing the envisaged improvements in tax administration-including a unified social security administration-and preserving the benefits of the recent VAT reform.

"Monetary policy continues to aim at achieving gradual disinflation and averting excessive real appreciation of the currency. The authorities are aware that scope for further interest rate cuts is now constrained by wage developments and the need to keep a strong external position. To prevent the build-up of excessive vulnerabilities, close monitoring of the rapid growth of credit, particularly that in foreign-currency, remains warranted.

"The authorities are restructuring a group of large loss-making state-owned enterprises to strengthen their financial position and with a view to their eventual privatization. This is a commendable effort, and the government would be well advised to extend its scope in the future. Perseverance in improving energy utilities' collection rates is also crucial for improving stateowned enterprises' financial balances and enforcing hard-budget constraints throughout the economy. Progress in energy sector privatization, particularly of gas and electricity distribution companies, will also be key for improving efficiency. In the banking sector, the government is committed to sell a minority share in the largest bank, BCR, and to complete the bank's privatization as soon as market conditions improve," Ms. Krueger said. 


\section{Statement by Yuriy Yakusha, Alternate Executive Director for Romania April 25, 2003}

On behalf of my Romanian authorities, I would like to thank staff for their frankness in discussions and constructive policy advice. The staff report presents a well balanced assessment of recent economic development.

Over the past two years, Romania has achieved commendable progress in macroeconomic stabilization, reflecting the determined implementation by the authorities of policy measures. Despite the global slowdown, macroeconomic developments have continued to be favorable and fully in line with program targets. The Romanian economy has experienced rapid growth, among the highest in the region, driven by vigorous exports, as well as by investment. The recent data show that January-February 2003 exports were 38.7 percent higher than in the same period of 2002. Fiscal adjustment and control over wages in the public sector, have helped to consolidate Romania's fiscal and external account sustainability. Inflation declined faster than targeted (from 30 percent at end-2001 to 18 percent at end-December 2002, and 17.1 percent at end-March 2003), and the external position strengthened more than expected. The current account deficit reached 3.4 percent of GDP at end-2002 and the increase in official reserves has been significantly stronger than estimated under the program, with a comfortable level of more than 4 months of prospective imports of goods and services. Total public and publicly guaranteed debt has remained under 30 percent of GDP, while domestic public debt was reduced to 7.2 percent of GDP. Access to the international market has been consolidated and the 10-year Eurobond issue was awarded by Euromoney as Best Sovereign Deal Emerging Europe in 2002. Romania's impressive macroeconomic performance has been recognized by the recent credit rating upgrade by Standard \&Poor's to BB minus, confirming the positive outlook. Moreover, Romania's success in macroeconomic stabilization and economic restructuring in the past few years led to the US's recognition of the country as a functioning market economy.

All prior actions for the third review under the SBA and additional measures for correcting the slippages on some structural areas were observed. At the same time, Romania made further progress towards its goal of EU accession. The whole economic strategy should be seen in the context of the aim to assume the obligations of EU membership by 2007, as envisaged at the Copenhagen summit last December. In this respect, negotiations on all chapters of the acquis communautaire had been opened, 17 of them being provisionally closed.

Despite encouraging macroeconomic achievements, the Romanian authorities are fully aware of the remaining weaknesses and the challenging reform agenda, mainly in areas of enterprise restructuring, financial discipline and privatization. Therefore, the Romanian authorities wish to restate their determination to continue with implementation of the economic policies aimed at ensuring long-term sustainable economic growth. 
Fiscal consolidation has been at the centre of Romania's adjustment policies. 2002 proved to be a successful year, as the general government deficit dropped to 2.6 percent of GDP, compared with the 3.0 percent targeted by the 2002 budget law and to the deficit of 3.2 percent in 2001 . Lower debt servicing costs and energy price adjustments, which reduced the quasi-fiscal losses, contributed to this result. In 2003, fiscal policy will continue to support disinflation. In this respect, preliminary data for the first quarter of this year suggest that the budget deficit remained well below the program target.

Special attention is given to improving revenue performance. The new VAT and profit tax laws have limited the number of tax exemptions and distortionary tax incentives, and a comprehensive reform of the tax administration is under way. On the expenditure side, the authorities have made efforts to create scope for increasing capital expenditure and improving the social safety net.

As regards the fuel excise taxes, the authorities are committed to pay special attention to eliminating the shortfalls on these collections. The revenue shottfalls in the fuel excise taxes are mainly caused by the high level of tax avoidance. The authorities, therefore, decided that the best way to increase these revenues is not to raise the tax level (penalizing good payers), but to increase payment discipline by a new package of the following measures (which should improve control and strengthen the payment discipline in this area) :

(i) Limiting the boarder/custom points for the transit of fuel imports to only five. Thus, controls will be strengthened and products carefully certified; (ii) Depositing all fuel quantities in special warehouses for better quality and movement control of all the products to counter tax cvasion; and, (iii) Introducing more severe penalties for nonpayers (criminal instead of financial penalties). The authorities are fully determined to restore the fuel excise value in curo as of January 2003, if the increase in excise collections would not be significant enough after 45 days of this new control regime. In addition to this package, the authorities decided to adjust the unified end-user price for natural gas to the cquivalent of US\$97-99 per one thousand cubic meters as of July 1, 2003, instead of the program's target of US\$93, in order to compensate for higher import costs. At the same time, the Romanian authorities remain firmly committed to refrain from any tax cuts in 2003, other than the 5 percentage point reduction in payroll taxes in January, as mentioned in the MEFP and they stand ready to identify additional steps, if the fiscal target is endangered.

Wage policy has been strengthened in the last part of 2002, and the end-December revised program target was missed by a negligible margin of 0.2 percent. As a result, wages in state-owned enterprises increased in line with the private sector, by about $3 \frac{3}{4}$ percent in real terms for the whole year. The 2003 budgets of the monitored SOEs were timely approved in December 2002 and the wage discipline has become more credible, supported by the government's strong determination to avoid any slippages in this area. The authorities have notified monitored SOEs that the wage bills would have to be adjusted in case of outsourcing of employees, and imposed a partial hiring freeze. They are carefully monitoring wage developments and are ready to implement additional measures, should spillover effects from the minimum wage increase in Jamuary 2003 prove larger than expected. 
Monetary policy is pursuing gradual disinflation without putting external competitiveness at risk. Under these terms, the NBR is aiming at a 1-3 percent annual real effective appreciation as a disinflation anchor. The NBR has pursued a prudent path reducing its key reference rate, which has also resulted in a gradual reduction of real interest rates. The development of the exchange rate during the period has been within the projected path. The NBR has limited its interventions on the market, while maintaining its net buyer position. In January 1, 2003, the NBR also started a gradual process of capital account liberalization. The overall volume of purchases in the first quarter of 2003 was almost four times smaller than during the same period of 2002 ; while supply on domestic foreign exchange markets has continued to increase, demand from nonbanker clients has been outstripping it to the tune of about Euro 300 million in the first quarter of 2003. Liquidity sterilization has been almost complete with the 12-month real M2 growth decline to 14.6 percent in March 2003 from 17.3 percent in December 2002.

Moreover, steps have already been taken to slow down the rapid expansion in foreign currency denominated credits. The reserve requirements in lei and in the foreign exchange were differentiated in October 2002 to 18 and 25 percent, respectively and the central bank bas continued to exercise close supervision of the most active lenders. In the first months of this year the volume of new lending in domestic currency surpassed that in foreign currency. The new regulation on loan classification and provisioning in force from January 2003 encourages commercial banks to closely monitor their portfolio with a view to reassess credit risk stemming from rapid credit expansion. It also helps that banking legislation is now at a high degree of alignment with international standards. The authorities consider Romania's participation in FSAP, which started in March 2003 as useful, both with regard to the general assessment of the financial system, as well as to defining the areas of concern.

Structural reforms remain the key challenge for Romania, Progress is being made in this area, but the authorities are fully aware that a significantly faster pace of restructuring is needed, in order to sustain macroeconomic stabilization gains and to ensure the full functioning of a market able to cope with competitive pressures within the EU market. Therefore, a more decisive approach has been taken this year to strengthen financial discipline, private sector development, and improve the business climate.

The private sector has continued to grow; an additional 6 large companies were privatized in the first quarter of 2003 and another 7 companies are in advanced negotiations. In considering the relatively slow pace of privatization so far, the unfavorable market conditions and the limited attractiveness of some large enterprises should be taken into consideration. Many large companies could not be easily divested because of their inefficiency and overstaffing. Therefore, in order to make them more attractive for privatization, restructuring plans have been approved and steps have been taken to significantly reduce employment in 22 state-owned companies and several mines. These companies are the largest loss makers and nonpayers of utilities' bills. This April, more than 6,000 layoffs took place (as a prior action), while a hiring freeze in these companies is in effect until year-end. The restructuring process will continue, involving at least an additional 
18,000 layoffs, as an important step. The proposed severance payments, after negotiations with trade unions, have ensured a smoother transition to efficiency.

Steps for privatization of the oil company Petrom and of the two electricity distribution companies are within the target. The authorities were so far unable to sell the largest commercial bank (BCR) due to low interest from strategic investors and therefore, decided to start the process by selling 25 percent of BCR's stake to EBRD and IFC. The negotiations with the two financial institutions are underway, and the selling contracts will be signed by end-July.

The weak financial discipline, mainly in the energy sector, has been addressed by significant price adjustments to cost-efficiency levels, and disconnections to the larger nonpayers have been implemented.

An anti-corruption legislative package was approved by the Parliament in March and is to become effective in the next 15 days, to address this issue more forcefully and to support efforts in improving business confidence.

In view of the above, the completion of the third review is deserved. In addition, we are requesting an extension of the program, which would help to consolidate the positive performance, strengthen structural reforms and address weaknesses in order to ensure the necessary steps for EU accession by 2007. 